\title{
HYPERELLIPTIC AND TRIGONAL FANO THREEFOLDS
}

\author{
IVAN CHELTSOV, CONSTANTIN SHRAMOV, VICTOR PRZYJALKOWSKI \\ to the 65-th anniversary of Vasily Iskovskikh
}

\begin{abstract}
We classify Fano 3-folds with canonical Gorenstein singularities whose anticanonical linear system has no base points but does not give an embedding, and we classify anticanonically embedded Fano 3-folds with canonical Gorenstein singularities which are not an intersection of quadrics.
\end{abstract}

\section{INTRODUCTION.}

The biregular classification of 3 -folds whose curve sections are canonical curves was considered by G. Fano in 31, 32, 33, 334. In the smooth case the hyperplane sections of such 3-folds must be K3 surfaces due to adjunction. So the natural generalization of 3 -folds studied by G. Fano are 3-folds containing an ample Cartier divisor, which is a K3 surface with at most Du Val singularities. Actually, except the generalized cones (usual cones in the very ample case) the latter 3-folds are Fano 3-folds with canonical Gorenstein singularities $^{1}$ (see [108, [100], [50, [12, [13]).

The classification of smooth Fano 3-folds was obtained in [109, [55, [56, 88, [92, [91, [89], where 105 families of Fano 3-folds were found (see 68]). Moreover, every Fano 3-fold with terminal Gorenstein singularities is a deformation of a smooth one due to [93, but there are Fano 3-folds with canonical Gorenstein singularities that can not be deformed into smooth Fano 3 -folds, e.g. the weighted projective spaces $\mathbb{P}\left(1^{3}, 3\right)$ and $\mathbb{P}\left(1^{2}, 4,6\right)$ (see [27, [35]).

Today the classification of Fano 3-folds with canonical Gorenstein singularities is far from being complete (cf. [91]), but there are 4 important steps that are already done. The first one is the following result proved in [108, and [100] (cf. [1], 2]).

Theorem 1.1. Let $X$ be a Fano 3-fold with canonical Gorenstein singularities, i.e. the anticanonical divisor $-K_{X}$ is an ample Cartier divisor, and $S$ be a sufficiently general surface in the linear system $\left|-K_{X}\right|$. Then $S$ has at most Du Val singularities.

The second step is the following result proved in 91, which is a natural generalization of the classification of smooth Fano 3 -folds with Picard group $\mathbb{Z}$ obtained in [55], [56].

Theorem 1.2. Let $X$ be a Fano 3-fold with canonical Gorenstein singularities, such that the linear system $\left|-K_{X}\right|$ does not have a movable decomposition, i.e. the divisor $-K_{X}$ is not rationally equivalent $A+B$, where $A$ and $B$ are Weil divisors whose complete linear systems $|A|$ and $|B|$ have positive dimension. Then $X$ is one of the following 3-folds:

- a hypersurface of degree 6 in $\mathbb{P}\left(1^{4}, 3\right)$, and $-K_{X}^{3}=2$;

- a complete intersection of a quadric cone and a quartic in $\mathbb{P}\left(1^{5}, 2\right)$, and $-K_{X}^{3}=4$;

- a quartic hypersurface in $\mathbb{P}^{4}$, and $-K_{X}^{3}=4$;

The work was partially supported by RFFI grant $04-01-00613$.

${ }^{1}$ Canonical Gorenstein singularities are exactly rational Gorenstien singularities (see [29]). 
- a complete intersection of a quadric and a cubic in $\mathbb{P}^{5}$, and $-K_{X}^{3}=6$;

- a complete intersection of three quadrics in $\mathbb{P}^{6}$, and $-K_{X}^{3}=8$;

- an intersection of the Grassmannian $G(1,4) \subset \mathbb{P}^{9}$ with a linear subspace of codimension 2 and a quadric, and $-K_{X}^{3}=10$;

- an intersection of the orthogonal Grassmannian $O G(5,10) \subset \mathbb{P}^{15}$ with a linear subspace of codimension 7 , and $-K_{X}^{3}=12$;

- an intersection of the Grassmannian $G(2,6) \subset \mathbb{P}^{14}$ with a linear subspace of codimension 5 , and $-K_{X}^{3}=14$;

- an intersection of the symplectic Grassmannian $L G(3,6) \subset \mathbb{P}^{13}$ with a linear subspace of codimension 3 , and $-K_{X}^{3}=16$;

- an intersection of the $G_{2}$-homogeneous space $\Sigma \subset \mathbb{P}^{13}$ with a linear subspace of codimension 2 (see Example 5.2.2 in 68]), and $-K_{X}^{3}=18$;

- a Mukai-Umemura 3-fold $V_{22} \subset \mathbb{P}^{13}$ (see [92, 39], 68]), and $-K_{X}^{3}=22$.

The third step is the following boundness result proved in 94].

Theorem 1.3. Let $X$ be a Fano 3-fold with canonical Gorenstein singularities, i.e. the anticanonical divisor $-K_{X}$ is an ample Cartier divisor. Then $-K_{X}^{3} \leq 72$ and the equality implies that either $X \cong \mathbb{P}\left(1^{3}, 3\right)$ or $X \cong \mathbb{P}\left(1^{2}, 4,6\right)$.

The forth step is the following result proved in [49].

Theorem 1.4. Let $X$ be a Fano 3-fold with canonical Gorenstein singularities such that the base locus of $\left|-K_{X}\right|$ is not empty. Then $X$ is one of the following 3-folds:

$\left(B_{1}\right)$ a complete intersection of a quadric cone and a sextic in $\mathbb{P}\left(1^{4}, 2,3\right)$, and $-K_{X}^{3}=2$;

$\left(B_{2}\right)$ a blow up of a sextic in $\mathbb{P}\left(1^{3}, 2,3\right)$ along a curve of arithmetic genus 1 , and $-K_{X}^{3}=$ 4 ;

$\left(B_{3}\right) S_{1} \times \mathbb{P}^{1}$, where $S_{1}$ is a del Pezzo surface of degree 1 with Du Val singularities, and $-K_{X}^{3}=6$;

$\left(B_{4}\right)$ an anticanonical model of a blow up of a hypersurface of degree 12 in $\mathbb{P}\left(1^{2}, 4^{2}, 6\right)$ in a cDV point, and $-K_{X}^{3}=6$.

The purpose of this paper is to prove the following two results.

Theorem 1.5. Let $X$ be a Fano variety with canonical Gorenstein singularities, such that the linear system $\left|-K_{X}\right|$ has no base points, but the induced morphism $\phi_{\left|-K_{X}\right|}$ is not an embedding. Then the 3-fold $X$ is one of the following 47 Fano 3-folds:

$\left(H_{1}\right)$ a hypersurface of degree 6 in $\mathbb{P}\left(1^{3}, 2,3\right)$, and $-K_{X}^{3}=8$;

$\left(\mathrm{H}_{2}\right)$ a hypersurface of degree 6 in $\mathbb{P}\left(1^{4}, 3\right)$, and $-K_{X}^{3}=2$;

$\left(H_{3}\right)$ a complete intersection of a quadric cone and a quartic in $\mathbb{P}\left(1^{5}, 2\right)$, and $-K_{X}^{3}=4$;

- an anticanonical model of a weak Fano 3-fold $V$ with canonical Gorenstein singularities, i.e. $-K_{V}$ is nef and big Cartier divisor and $\phi_{\left|-r K_{V}\right|}(V)=X$ for $r \gg 0$, such that $V$ is a double cover of the rational scroll $\mathbb{F}\left(d_{1}, d_{2}, d_{3}\right)=$ $\operatorname{Proj}\left(\oplus_{i=1}^{3} \mathcal{O}_{\mathbb{P}^{1}}\left(d_{i}\right)\right)$ branched over a divisor that is rationally equivalent to a divisor $4 M+2\left(2-\sum_{i=1}^{3} d_{i}\right) L$, where $M$ is a class of a tautological line bundle on $\mathbb{F}\left(d_{1}, d_{2}, d_{3}\right)$ and $L$ is a class of a fiber of the projection of the scroll $\mathbb{F}\left(d_{1}, d_{2}, d_{3}\right)$ to $\mathbb{P}^{1}$, such that the following cases hold:

$\left(H_{4}\right) d_{1}=1, d_{2}=1, d_{3}=1$, and $-K_{X}^{3}=6$;

$\left(H_{5}\right) d_{1}=2, d_{2}=1, d_{3}=0$, and $-K_{X}^{3}=6$;

$\left(H_{6}\right) d_{1}=2, d_{2}=1, d_{3}=1$, and $-K_{X}^{3}=8$;

$\left(H_{7}\right) d_{1}=2, d_{2}=2, d_{3}=0$, and $-K_{X}^{3}=8$; 
$\left(H_{8}\right) d_{1}=2, d_{2}=2, d_{3}=1$, and $-K_{X}^{3}=10$;

$\left(H_{9}\right) d_{1}=2, d_{2}=2, d_{3}=2$, and $-K_{X}^{3}=12$;

$\left(H_{10}\right) d_{1}=3, d_{2}=0, d_{3}=0$, and $-K_{X}^{3}=6$;

$\left(H_{11}\right) d_{1}=3, d_{2}=1, d_{3}=0$, and $-K_{X}^{3}=8$;

$\left(H_{12}\right) d_{1}=3, d_{2}=1, d_{3}=1$, and $-K_{X}^{3}=10$;

$\left(H_{13}\right) d_{1}=3, d_{2}=2, d_{3}=0$, and $-K_{X}^{3}=10$;

$\left(H_{14}\right) d_{1}=3, d_{2}=2, d_{3}=1$, and $-K_{X}^{3}=12$;

$\left(H_{15}\right) d_{1}=3, d_{2}=3, d_{3}=0$, and $-K_{X}^{3}=12$;

$\left(H_{16}\right) d_{1}=3, d_{2}=3, d_{3}=1$, and $-K_{X}^{3}=14$;

$\left(H_{17}\right) d_{1}=4, d_{2}=0, d_{3}=0$, and $-K_{X}^{3}=8$;

$\left(H_{18}\right) d_{1}=4, d_{2}=1, d_{3}=0$, and $-K_{X}^{3}=10$;

$\left(H_{19}\right) d_{1}=4, d_{2}=2, d_{3}=0$, and $-K_{X}^{3}=12$;

$\left(H_{20}\right) d_{1}=4, d_{2}=2, d_{3}=1$, and $-K_{X}^{3}=14$;

$\left(H_{21}\right) d_{1}=4, d_{2}=3, d_{3}=0$, and $-K_{X}^{3}=14$;

$\left(H_{22}\right) d_{1}=4, d_{2}=3, d_{3}=1$, and $-K_{X}^{3}=16$;

$\left(H_{23}\right) d_{1}=4, d_{2}=4, d_{3}=0$, and $-K_{X}^{3}=16$;

$\left(H_{24}\right) d_{1}=5, d_{2}=1, d_{3}=0$, and $-K_{X}^{3}=12$;

$\left(H_{25}\right) d_{1}=5, d_{2}=2, d_{3}=0$, and $-K_{X}^{3}=14$;

$\left(H_{26}\right) d_{1}=5, d_{2}=3, d_{3}=0$, and $-K_{X}^{3}=16$;

$\left(H_{27}\right) d_{1}=5, d_{2}=3, d_{3}=1$, and $-K_{X}^{3}=18$;

$\left(H_{28}\right) d_{1}=5, d_{2}=4, d_{3}=0$, and $-K_{X}^{3}=18$;

$\left(H_{29}\right) d_{1}=5, d_{2}=4, d_{3}=1$, and $-K_{X}^{3}=20$;

$\left(H_{30}\right) d_{1}=6, d_{2}=2, d_{3}=0$, and $-K_{X}^{3}=16$;

$\left(H_{31}\right) d_{1}=6, d_{2}=3, d_{3}=0$, and $-K_{X}^{3}=18$;

$\left(H_{32}\right) d_{1}=6, d_{2}=4, d_{3}=0$, and $-K_{X}^{3}=20$;

$\left(H_{33}\right) d_{1}=6, d_{2}=4, d_{3}=1$, and $-K_{X}^{3}=22$;

$\left(H_{34}\right) d_{1}=6, d_{2}=5, d_{3}=0$, and $-K_{X}^{3}=22$;

$\left(H_{35}\right) d_{1}=7, d_{2}=3, d_{3}=0$, and $-K_{X}^{3}=20$;

$\left(H_{36}\right) d_{1}=7, d_{2}=4, d_{3}=0$, and $-K_{X}^{3}=22$;

$\left(H_{37}\right) d_{1}=7, d_{2}=5, d_{3}=0$, and $-K_{X}^{3}=24$;

$\left(H_{38}\right) d_{1}=7, d_{2}=5, d_{3}=1$, and $-K_{X}^{3}=26$;

$\left(H_{39}\right) d_{1}=8, d_{2}=4, d_{3}=0$, and $-K_{X}^{3}=24$;

$\left(H_{40}\right) d_{1}=8, d_{2}=5, d_{3}=0$, and $-K_{X}^{3}=26$;

$\left(H_{41}\right) d_{1}=8, d_{2}=6, d_{3}=0$, and $-K_{X}^{3}=28$;

$\left(H_{42}\right) d_{1}=9, d_{2}=5, d_{3}=0$, and $-K_{X}^{3}=28$;

$\left(H_{43}\right) d_{1}=9, d_{2}=6, d_{3}=0$, and $-K_{X}^{3}=30$;

$\left(H_{44}\right) d_{1}=10, d_{2}=6, d_{3}=0$, and $-K_{X}^{3}=32$;

$\left(H_{45}\right) d_{1}=10, d_{2}=7, d_{3}=0$, and $-K_{X}^{3}=34$;

$\left(H_{46}\right) d_{1}=11, d_{2}=7, d_{3}=0$, and $-K_{X}^{3}=36$;

$\left(H_{47}\right) d_{1}=12, d_{2}=8, d_{3}=0$, and $-K_{X}^{3}=40$.

Theorem 1.6. Let $X$ be a Fano 3-fold with canonical Gorenstein singularities, such that the linear system $\left|-K_{X}\right|$ has no base points and the induced morphism $\phi_{\left|-K_{X}\right|}$ is an embedding, but the anticanonical image $\phi_{\left|-K_{X}\right|}(X) \subset \mathbb{P}^{n}$ is not an intersection of quadrics, where $n=-\frac{K_{X}^{3}}{2}+2$. Then the 3-fold $X$ is one of the following 69 Fano 3-folds:

$\left(T_{1}\right)$ a quartic hypersurface in $\mathbb{P}^{4}$, and $-K_{X}^{3}=4$;

$\left(T_{2}\right)$ a complete intersection of a quadric and a cubic in $\mathbb{P}^{5}$, and $-K_{X}^{3}=6$; 
$\left(T_{3}\right)$ an anticanonical image of a weak Fano 3-fold $Y$ with canonical Gorenstein singularities, i.e. the divisor $-K_{Y}$ is a nef and big Cartier divisor and $\phi_{\left|-K_{Y}\right|}(Y)=X$, such that $Y$ is a divisor in the scroll $\operatorname{Proj}\left(\mathcal{O}_{\mathbb{P}^{2}}(2) \oplus \mathcal{O}_{\mathbb{P}^{2}} \oplus \mathcal{O}_{\mathbb{P}^{2}}\right)$ that is rationally equivalent to a divisor $2 T+F$, where $T$ is a class of a tautological line bundle on $\operatorname{Proj}\left(\mathcal{O}_{\mathbb{P}^{2}}(2) \oplus \mathcal{O}_{\mathbb{P}^{2}} \oplus \mathcal{O}_{\mathbb{P}^{2}}\right)$ and $F$ is pull back of $\mathcal{O}_{\mathbb{P}^{2}}(1)$ via the natural projection to $\mathbb{P}^{2}$, and $-K_{X}^{3}=10$;

- an anticanonical image of a weak Fano 3-fold $V$ with canonical Gorenstein singularities, i.e. the divisor $-K_{V}$ is a nef and big Cartier divisor and $\phi_{\left|-K_{V}\right|}(V)=X$, such that $V$ is a divisor in $\mathbb{F}\left(d_{1}, d_{2}, d_{3}, d_{4}\right)=\operatorname{Proj}\left(\oplus_{i=1}^{4} \mathcal{O}_{\mathbb{P}^{1}}\left(d_{i}\right)\right)$ that is rationally equivalent to a divisor $3 M+\left(2-\sum_{i=1}^{4} d_{i}\right) L$, where $M$ is a class of a tautological line bundle on the scroll $\mathbb{F}\left(d_{1}, d_{2}, d_{3}, d_{4}\right)$ and $L$ is a class of a fiber of the projection of $\mathbb{F}\left(d_{1}, d_{2}, d_{3}, d_{4}\right)$ to $\mathbb{P}^{1}$, such that the following cases hold:

$\left(T_{4}\right) d_{1}=1, d_{2}=1, d_{3}=1, d_{4}=0$, and $-K_{X}^{3}=8$;

$\left(T_{5}\right) d_{1}=1, d_{2}=1, d_{3}=1, d_{4}=1$, and $-K_{X}^{3}=10$;

$\left(T_{6}\right) d_{1}=2, d_{2}=1, d_{3}=0, d_{4}=0$, and $-K_{X}^{3}=8$;

$\left(T_{7}\right) d_{1}=2, d_{2}=1, d_{3}=1, d_{4}=0$, and $-K_{X}^{3}=10$;

$\left(T_{8}\right) d_{1}=2, d_{2}=1, d_{3}=1, d_{4}=1$, and $-K_{X}^{3}=12$;

$\left(T_{9}\right) d_{1}=2, d_{2}=2, d_{3}=0, d_{4}=0$, and $-K_{X}^{3}=10$;

$\left(T_{10}\right) d_{1}=2, d_{2}=2, d_{3}=1, d_{4}=0$, and $-K_{X}^{3}=12$;

$\left(T_{11}\right) d_{1}=2, d_{2}=2, d_{3}=1, d_{4}=1$, and $-K_{X}^{3}=14$;

$\left(T_{12}\right) d_{1}=2, d_{2}=2, d_{3}=2, d_{4}=0$, and $-K_{X}^{3}=14$;

$\left(T_{13}\right) d_{1}=2, d_{2}=2, d_{3}=2, d_{4}=1$, and $-K_{X}^{3}=16$;

$\left(T_{14}\right) d_{1}=2, d_{2}=2, d_{3}=2, d_{4}=2$, and $-K_{X}^{3}=18$;

$\left(T_{15}\right) d_{1}=3, d_{2}=1, d_{3}=0, d_{4}=0$, and $-K_{X}^{3}=10$;

$\left(T_{16}\right) d_{1}=3, d_{2}=1, d_{3}=1, d_{4}=0$, and $-K_{X}^{3}=12$;

$\left(T_{17}\right) d_{1}=3, d_{2}=2, d_{3}=0, d_{4}=0$, and $-K_{X}^{3}=12$;

$\left(T_{18}\right) d_{1}=3, d_{2}=2, d_{3}=1, d_{4}=0$, and $-K_{X}^{3}=14$;

$\left(T_{19}\right) d_{1}=3, d_{2}=2, d_{3}=1, d_{4}=1$, and $-K_{X}^{3}=16$;

$\left(T_{20}\right) d_{1}=3, d_{2}=2, d_{3}=2, d_{4}=0$, and $-K_{X}^{3}=16$;

$\left(T_{21}\right) d_{1}=3, d_{2}=2, d_{3}=2, d_{4}=1$, and $-K_{X}^{3}=18$;

$\left(T_{22}\right) d_{1}=3, d_{2}=3, d_{3}=1, d_{4}=0$, and $-K_{X}^{3}=16$;

$\left(T_{23}\right) d_{1}=3, d_{2}=3, d_{3}=2, d_{4}=0$, and $-K_{X}^{3}=18$;

$\left(T_{24}\right) d_{1}=3, d_{2}=3, d_{3}=2, d_{4}=1$, and $-K_{X}^{3}=20$;

$\left(T_{25}\right) d_{1}=4, d_{2}=1, d_{3}=0, d_{4}=0$, and $-K_{X}^{3}=12$;

$\left(T_{26}\right) d_{1}=4, d_{2}=2, d_{3}=0, d_{4}=0$, and $-K_{X}^{3}=14$;

$\left(T_{27}\right) d_{1}=4, d_{2}=2, d_{3}=1, d_{4}=0$, and $-K_{X}^{3}=16$;

$\left(T_{28}\right) d_{1}=4, d_{2}=2, d_{3}=1, d_{4}=1$, and $-K_{X}^{3}=18$;

$\left(T_{29}\right) d_{1}=4, d_{2}=2, d_{3}=2, d_{4}=0$, and $-K_{X}^{3}=18$;

$\left(T_{30}\right) d_{1}=4, d_{2}=3, d_{3}=1, d_{4}=0$, and $-K_{X}^{3}=18$;

$\left(T_{31}\right) d_{1}=4, d_{2}=3, d_{3}=2, d_{4}=0$, and $-K_{X}^{3}=20$;

$\left(T_{32}\right) d_{1}=4, d_{2}=3, d_{3}=2, d_{4}=1$, and $-K_{X}^{3}=22$;

$\left(T_{33}\right) d_{1}=4, d_{2}=3, d_{3}=3, d_{4}=0$, and $-K_{X}^{3}=22$;

$\left(T_{34}\right) d_{1}=4, d_{2}=3, d_{3}=3, d_{4}=1$, and $-K_{X}^{3}=24$;

$\left(T_{35}\right) d_{1}=4, d_{2}=4, d_{3}=2, d_{4}=0$, and $-K_{X}^{3}=22$;

$\left(T_{36}\right) d_{1}=5, d_{2}=2, d_{3}=0, d_{4}=0$, and $-K_{X}^{3}=16$;

$\left(T_{37}\right) d_{1}=5, d_{2}=2, d_{3}=1, d_{4}=0$, and $-K_{X}^{3}=18$;

$\left(T_{38}\right) d_{1}=5, d_{2}=3, d_{3}=1, d_{4}=0$, and $-K_{X}^{3}=20$; 
$\left(T_{39}\right) d_{1}=5, d_{2}=3, d_{3}=2, d_{4}=0$, and $-K_{X}^{3}=22$;

$\left(T_{40}\right) d_{1}=5, d_{2}=3, d_{3}=2, d_{4}=1$, and $-K_{X}^{3}=24$;

$\left(T_{41}\right) d_{1}=5, d_{2}=3, d_{3}=3, d_{4}=0$, and $-K_{X}^{3}=24$;

$\left(T_{42}\right) d_{1}=5, d_{2}=4, d_{3}=2, d_{4}=0$, and $-K_{X}^{3}=24$;

$\left(T_{43}\right) d_{1}=5, d_{2}=4, d_{3}=3, d_{4}=0$, and $-K_{X}^{3}=26$;

$\left(T_{44}\right) d_{1}=5, d_{2}=4, d_{3}=3, d_{4}=1$, and $-K_{X}^{3}=28$;

$\left(T_{45}\right) d_{1}=6, d_{2}=2, d_{3}=0, d_{4}=0$, and $-K_{X}^{3}=18$;

$\left(T_{46}\right) d_{1}=6, d_{2}=3, d_{3}=1, d_{4}=0$, and $-K_{X}^{3}=22$;

$\left(T_{47}\right) d_{1}=6, d_{2}=3, d_{3}=2, d_{4}=0$, and $-K_{X}^{3}=24$;

$\left(T_{48}\right) d_{1}=6, d_{2}=4, d_{3}=2, d_{4}=0$, and $-K_{X}^{3}=26$;

$\left(T_{49}\right) d_{1}=6, d_{2}=4, d_{3}=3, d_{4}=0$, and $-K_{X}^{3}=28$;

$\left(T_{50}\right) d_{1}=6, d_{2}=4, d_{3}=3, d_{4}=1$, and $-K_{X}^{3}=30$;

$\left(T_{51}\right) d_{1}=6, d_{2}=4, d_{3}=4, d_{4}=0$, and $-K_{X}^{3}=30$;

$\left(T_{52}\right) d_{1}=6, d_{2}=5, d_{3}=3, d_{4}=0$, and $-K_{X}^{3}=30$;

$\left(T_{53}\right) d_{1}=7, d_{2}=3, d_{3}=1, d_{4}=0$, and $-K_{X}^{3}=24$;

$\left(T_{54}\right) d_{1}=7, d_{2}=4, d_{3}=2, d_{4}=0$, and $-K_{X}^{3}=28$;

$\left(T_{55}\right) d_{1}=7, d_{2}=4, d_{3}=3, d_{4}=0$, and $-K_{X}^{3}=30$;

$\left(T_{56}\right) d_{1}=7, d_{2}=5, d_{3}=3, d_{4}=0$, and $-K_{X}^{3}=32$;

$\left(T_{57}\right) d_{1}=7, d_{2}=5, d_{3}=4, d_{4}=0$, and $-K_{X}^{3}=34$;

$\left(T_{58}\right) d_{1}=7, d_{2}=5, d_{3}=4, d_{4}=1$, and $-K_{X}^{3}=36$;

$\left(T_{59}\right) d_{1}=8, d_{2}=4, d_{3}=2, d_{4}=0$, and $-K_{X}^{3}=30$;

$\left(T_{60}\right) d_{1}=8, d_{2}=5, d_{3}=3, d_{4}=0$, and $-K_{X}^{3}=34$;

$\left(T_{61}\right) d_{1}=8, d_{2}=5, d_{3}=4, d_{4}=0$, and $-K_{X}^{3}=36$;

$\left(T_{62}\right) d_{1}=8, d_{2}=6, d_{3}=4, d_{4}=0$, and $-K_{X}^{3}=38$;

$\left(T_{63}\right) d_{1}=9, d_{2}=5, d_{3}=3, d_{4}=0$, and $-K_{X}^{3}=36$;

$\left(T_{64}\right) d_{1}=9, d_{2}=6, d_{3}=4, d_{4}=0$, and $-K_{X}^{3}=40$;

$\left(T_{65}\right) d_{1}=9, d_{2}=6, d_{3}=5, d_{4}=0$, and $-K_{X}^{3}=42$;

$\left(T_{66}\right) d_{1}=10, d_{2}=6, d_{3}=4, d_{4}=0$, and $-K_{X}^{3}=42$;

$\left(T_{67}\right) d_{1}=10, d_{2}=7, d_{3}=5, d_{4}=0$, and $-K_{X}^{3}=46$;

$\left(T_{68}\right) d_{1}=11, d_{2}=7, d_{3}=5, d_{4}=0$, and $-K_{X}^{3}=48$;

$\left(T_{69}\right) d_{1}=12, d_{2}=8, d_{3}=6, d_{4}=0$, and $-K_{X}^{3}=54$.

In the smooth case Theorems 1.4, 1.5, 1.6 were proved by V. Iskovskikh (see [58, 61]).

Remark 1.7. For any Fano 3-fold with canonical Gorenstein singularities $X$ there is a birational morphism, so-called terminal modification, $f: V \rightarrow X$ such that $K_{V} \sim f^{*}\left(K_{V}\right)$ and $V$ has terminal Gorenstein singularities. The existence of $f$ follows from Minimal Model Program and the contraction theorem (see [72]). On the other hand, for any weak Fano 3 -fold $V$ with canonical Gorenstein singularities, i.e. $-K_{V}$ is nef and big, the contraction theorem implies the existence of the birational morphism $f: V \rightarrow X$ such that $X$ is a Fano 3-fold with canonical Gorenstein singularities.

In the following we will use the symbols $B_{k}, H_{i}$ and $T_{j}$ as names of the corresponding Fano 3-folds with canonical Gorenstein singularities from Theorems 1.4, 1.5 and 1.6 respectively. The 3 -folds $H_{i}$ and $T_{j}$ are called hyperelliptic and trigonal respectively (sometimes excluding the 3 -fold $T_{3}$, but we will call $T_{3}$ trigonal as well).

Remark 1.8. The 3-fold $H_{1}$ is a double cover of a cone over the Veronese surface, $H_{2}$ is a double cover of $\mathbb{P}^{3}$ ramified in a sextic surface. The 3 -fold $H_{3}$ is a double cover of a possibly singular quadric 3 -fold, $H_{4}$ is a double cover of $\mathbb{P}^{1} \times \mathbb{P}^{2}$ branched over a divisor 
of bi-degree $(2,4)$. The 3 -fold $H_{6}$ is a blow up of a hypersurface of degree 4 in $\mathbb{P}\left(1^{4}, 2\right)$, i.e. a double cover of $\mathbb{P}^{3}$ branched over a quartic surface, along the intersection of two different divisors in half-anticanonical linear system. The 3 -fold $H_{9}$ is a product $\mathbb{P}^{1} \times S_{2}$, where $S_{2}$ is a del Pezzo surface of degree 2 with Du Val singularities. The 3 -fold $H_{10}$ is a hypersurface in $\mathbb{P}\left(1^{2}, 3^{2}, 5\right)$ of degree 10 , the 3 -fold $H_{17}$ is a hypersurface in $\mathbb{P}\left(1^{2}, 4^{2}, 6\right)$ of degree 12 . The 3 -fold $T_{3}$ is a hypersurface in $\mathbb{P}\left(1^{3}, 2^{2}\right)$ of degree 5 , the 3 -fold $T_{5}$ is a divisor of bi-degree $(1,3)$ on $\mathbb{P}^{1} \times \mathbb{P}^{3}$, and the 3 -fold $T_{8}$ is a blow up of a plane cubic curve on a cubic 3 -fold in $\mathbb{P}^{4}$. The 3 -fold $T_{14}$ is a product $\mathbb{P}^{1} \times S_{3}$, where $S_{3}$ is a cubic surface in $\mathbb{P}^{3}$ with Du Val singularities.

Remark 1.9. The 3 -folds $H_{1}, H_{2}, H_{3}, H_{4}, H_{6}, H_{9}, T_{1}, T_{2}, T_{5}, T_{8}$ and $T_{14}$ are the only 3 -folds among the 3 -folds $H_{i}$ and $T_{j}$ that can be chosen smooth (see [58, 68]).

The 3 -folds $H_{i}$ and $T_{j}$ are rationally connected (see [75]). Moreover, the majority of 3 -folds $H_{i}$ and $T_{j}$ must be rational, but some of them are definitely not. For example, it is well known that the sufficiently general 3-folds $H_{1}, H_{2}, H_{3}, H_{4}, H_{6}, T_{1}, T_{2}$ and $T_{8}$ are not rational (see 67], 21], 8], 118, [59, 6], [78]). Moreover, their non-rationality can be proved in the smooth and some singular cases (see [112, [113, 114, 115, [96, [97, [98, 45], 44, 22., 23], 46, 86, 47], 18]), but all of them may be rational in singular case as well (cf. [69]). On the other hand, it is clear that the 3 -folds $H_{9}, T_{5}$ and $T_{14}$ are rational due to Remark 1.8. In this paper we will prove the following result.

Proposition 1.10. The 3-fold $H_{i}$ is rational for $i \in\{8,9,22,26,28,29,31, \ldots, 47\}$ and the 3-fold $T_{j}$ is rational for $j \in\{5,10,11,12,13,14,17, \ldots, 69\}$. On the other hand, sufficiently general 3-folds $H_{i}$ and $T_{j}$ are non-rational for $i \leq 7$ and $j \in\{1,2,3,4,6,7,8,9\}$.

There are birational relations between some 3 -folds $H_{i}, T_{j}$ and $B_{k}$. The simplest one is a projection from a cDV point, i.e. an anticanonical model of a blow up of a cDV point on one of the 3 -folds $H_{i}$ or $T_{j}$ of anticanonical degree $d \geq 4$, which must be one of the 3 -folds $H_{i}, T_{j}$ or $B_{k}$ of anticanonical degree $d-2$. For example, $B_{4}$ is birational to $H_{17}$, the 3 -fold $H_{5}$ is birational to $H_{1}$ with a cDV point (cf. Lemma 3.4 in [45]), the 3-folds $T_{1}$ and $T_{2}$ having a cDV point are birational to the singular 3 -folds $H_{2}$ and $T_{1}$ respectively. However, there are many non-obvious birational transformations of the 3 -folds $H_{i}$ and $T_{j}$.

Example 1.11. In the notations of from Theorem 1.6 let $X$ be a sufficiently general 3-fold $T_{7}$ and $V$ be a corresponding weak Fano 3 -fold $V \subset \mathbb{F}(2,1,1,0)$. Then $V$ is smooth (see the proof of Theorem [1.6) and $-K_{V}$ has trivial intersection only with one smooth rational curve $Y_{4} \subset \mathbb{F}(2,1,1,0)$ (cf. Corollary 2.20). Thus, birational morphism $\phi_{\left|-K_{V}\right|}: V \rightarrow X$ contracts the curve $Y_{4}$ to an ordinary double point of $X$. Let $f: V \rightarrow \tilde{V}$ be a flop in the curve $Y_{4}$. Then there is a birational morphism $g: \tilde{V} \rightarrow Y$ such that $Y$ is a smooth Fano 3-fold of index 2 and degree 2, i.e. a quartic double solid (see [19], 20]). Therefore, there is a double cover $\pi: Y \rightarrow \mathbb{P}^{3}$ branched over a smooth quartic surface $S \subset \mathbb{P}^{3}$. Moreover, birational morphism $g$ is a blow up of the smooth rational curve $C \subset Y$ such that $-K_{Y} \cdot C=2$. It is easy to see that all the constructions of all described birational maps are reversible, i.e. for a double cover $\pi: Y \rightarrow \mathbb{P}^{3}$ branched over a smooth quartic surface $S \subset \mathbb{P}^{3}$ and a smooth rational curve $C \subset Y$ with $-K_{Y} \cdot C=2$ one can construct the corresponding Fano 3-fold $T_{7}$ (see $§ 4.4 .1$ in [10]).

There are only two Fano 3-folds with canonical Gorenstein singularities whose anticanonical divisor is divisible by an integer greater than 2, i.e. $\mathbb{P}^{3}$ and a quadric 3-fold $Q \subset \mathbb{P}^{4}$. Fano 3 -folds with canonical Gorenstein singularities whose anticanonical divisor 
is divisible by 2 are called del Pezzo 3-folds (see Theorem 3.3.1 in [68]). It is easy to check or to prove explicitly that the only del Pezzo 3-fold among 3 -folds $H_{i}$ and $T_{j}$ is $H_{1}$, which is also confirmed by the classification of del Pezzo 3-folds (see [37, 38, 11], 105]).

Remark 1.12. The 3-folds $H_{i}$ and $T_{j}$ are naturally birational to a del Pezzo fibration of degree 2 and 3 respectively except the following cases: $H_{1}, H_{2}, H_{3}, T_{1}, T_{2}$ and $T_{3}$. On the other hand, the sufficiently general 3-folds $H_{1}, H_{2}, H_{3}, T_{1}, T_{2}$ are not birational to any del Pezzo fibration of degree 2 and 3 (see [67, [59, [97, [22], 46], 47]).

Remark 1.13. It is well known that 3-folds with a pencil of del Pezzo surfaces of degree 2 and 3 are unirational (see [80, 81, 82]). Therefore, the 3 -folds $H_{i}$ and $T_{j}$ are unirational for $i \geq 4$ and $j \geq 4$. On the other hand, the 3 -fold $H_{3}$ is known to be unirational (see [103, [59]). The unirationality of $T_{3}$ is implied by the proof of Proposition [5.5, i.e. the 3 -fold $T_{3}$ is birational to a conic bundle with a rational multisection. The 3 -fold $T_{2}$ is unirational as well (see [30], [103], [117], 59]). However, it is still unknown whether a general quartic 3 -fold $T_{1}$ is unirational or not. Nevertheless, there are few known examples of smooth unirational quartic 3-folds in [106, 67], [59, [84. Unfortunately, nothing is known about the unirationality of 3-folds $H_{1}$ and $H_{2}$ in the smooth case. Moreover, the sufficienly general 3 -fold $H_{2}$ is expected to be non-unirational (cf. Conjecture 4.1.6 in [77]).

The authors are very grateful to A. Corti, M. Grinenko, V. Iskovskikh, S. Kudryavtsev, V. Kulikov, Yu. Prokhorov, A. Pukhlikov, V. Shokurov, D. Stepanov and N. Zak for fruitful conversations.

In the following all varieties are assumed to be projective, normal, and defined over the field $\mathbb{C}$.

\section{Preliminaries.}

The following result is Proposition 3.1.6 of [76].

Proposition 2.1. Let $\rho: V \rightarrow X$ be a finite morphism, $D_{X}$ be an effective $\mathbb{Q}$-divisor on $X$ and $D_{V}=\rho^{*}\left(D_{X}\right)-K_{V / X}$, i.e. $K_{V}+D_{V}=\rho^{*}\left(K_{X}+D_{X}\right)$. Then the singularities of the log pair $\left(V, D_{V}\right)$ are Kawamata log terminal (see [72], 76]) if and only if the singularities of the log pair $\left(X, D_{X}\right)$ are Kawamata log terminal.

However, in the case when canonical divisor is a Cartier divisor Kawamata log terminal singularities are canonical. Hence, Proposition 2.1] implies the following result.

Corollary 2.2. Let $X$ be a smooth variety, $\rho: V \rightarrow X$ be a double cover branched over a reduced effective divisor $D \subset X$. Then $V$ has canonical singularities if and only if the log pair $\left(X, \frac{1}{2} D\right)$ has Kawamata log terminal singularities.

The following result is Theorem 4.5.1 of [76].

Theorem 2.3. Let $X$ be a smooth variety, $\mathcal{H}$ be a linear system on $X$ whose base locus has codimension at least 2 , and $D$ be a sufficiently general divisor in $\mathcal{H}$. Suppose that for every point $x \in X$ there is $H \in \mathcal{H}$ such that the singularities of $(X, H)$ are canonical in the neighbourhood of $x$. Then the singularities of the log pair $(X, D)$ are canonical.

The following result is Theorem 7.9 of [76] and is due to [11]. 
Theorem 2.4. Let $X$ be a normal variety such that $\omega_{X}$ is locally free and $S \subset X$ be an effective Cartier divisor. Then $S$ has canonical singularities if and only if the singularities of the log pair $(X, S)$ are canonical.

Hence, Theorems 2.3 and 2.4 imply the following result.

Corollary 2.5. Let $X$ be a smooth variety, $\mathcal{H}$ be a linear system on $X$ whose base locus has codimension at least 2 , and $D$ be a sufficiently general divisor in $\mathcal{H}$. Assume that for every point $x \in X$ there is a divisor $H \in \mathcal{H}$ such that the singularities of $H$ are canonical in the neighbourhood of the point $x$. Then $D$ has canonical singularities.

The following result is implied by Theorem 4.8 in [76]).

Theorem 2.6. Let $X$ be a smooth variety, $\mathcal{H}$ be a linear system on $X, D$ be a sufficiently general divisor in $\mathcal{H}$ and $\lambda \in \mathbb{Q} \cap[0,1)$. Assume that for every $x \in X$ there is $H \in \mathcal{H}$ such that the log pair $(X, \lambda H)$ has Kawamata log terminal singularities in the neighbourhood of the point $x$. Then the singularities of $(X, \lambda D)$ are Kawamata log terminal.

Thus, Corollary 2.2 and Theorem 2.6 imply the following result.

Corollary 2.7. Let $X$ be a smooth variety, $\mathcal{H}$ be a linear system on $X, D$ be a sufficiently general divisor in $\mathcal{H}$. Assume that for any point $O \in X$ there is an effective reduced divisor $H \in \mathcal{H}$ such that there is a double cover $\beta: Y \rightarrow X$ branched over $H \subset X$ and the variety $Y$ has canonical singularities in the neighbourhood of $\beta^{-1}(O)$. Let $\rho: V \rightarrow X$ be a double cover branched over $D \subset X$. Then $V$ has canonical singularities.

Remark 2.8. Actually, Corollary 2.5 easily implies Corollary 2.7. Indeed, in the notations of Corollary 2.7 let $B \subset X$ be a divisor such that $D \sim 2 B, U=\operatorname{Proj}\left(\mathcal{O}_{X} \oplus \mathcal{O}_{X}(B)\right)$, $M$ be a tautological line bundle on $U$, and $f: U \rightarrow X$ be a natural projection. Then $Y$ can be considered as a divisor on $U$ in the linear system $|2 M|$ such that $\beta=\left.f\right|_{Y}$. Without loss of generality we may assume that $\mathcal{H}=|H|$. Hence, we can identify $V$ with a sufficiently general divisor in the linear system $|2 M|$. However, the base locus of $|2 M|$ is contained in $Y \cap f^{-1}(H)$, because $2 S+f^{-1}(H) \sim 2 M$ and $S \cap Y=\emptyset$, where $S \sim M-f^{*}(B)$ is a negative section of $f: U \rightarrow X$. Therefore, the base locus of $|2 M|$ has codimension at least 2 and $V \in|2 M|$ has canonical singularities by Corollary 2.5.

Let us remind the following classical result (see [101, [7, 4], 90], 102]).

Theorem 2.9. Let $X$ be a normal algebraic surface and $O \in X$ be an isolated singular point such that the singularities of the surface $X$ are canonical in the neighbourhood of the point $O$, i.e. the point $O$ is a $D u$ Val singular point on $X$. Then $O \in X$ is a hypersurface quasi-homogeneous singular point on $X$ which is locally isomorphic to one of the following types of singularities $(0,0,0) \in \mathbb{C}^{3} \cong \operatorname{Spec}(\mathbb{C}[x, y, z])$ :

$\left(\mathbb{A}_{n}\right) x^{2}+y^{2}+z^{n+1}=0, \operatorname{wt}(x)=n+1, \operatorname{wt}(y)=n+1, \operatorname{wt}(z)=2$, and $n \geq 1$;

$\left(\mathbb{D}_{n}\right) x^{2}+y^{2} z+z^{n-1}=0, \operatorname{wt}(x)=n-1, \operatorname{wt}(y)=n-2, \operatorname{wt}(z)=2$, and $n \geq 4$;

$\left(\mathbb{E}_{6}\right) x^{2}+y^{3}+z^{4}=0, \operatorname{wt}(x)=6, \operatorname{wt}(y)=4, \operatorname{wt}(z)=3$;

$\left(\mathbb{E}_{7}\right) x^{2}+y^{3}+y z^{3}=0, \operatorname{wt}(x)=9, \operatorname{wt}(y)=6, \operatorname{wt}(z)=4$;

$\left(\mathbb{E}_{8}\right) x^{2}+y^{3}+z^{5}=0, \operatorname{wt}(x)=15, \operatorname{wt}(y)=10, \operatorname{wt}(z)=6$.

The following result is proved in $\S 12.3, \S 12.6$ and $\S 13.1$ of [4].

Theorem 2.10. Let $X \subset \mathbb{C}^{3} \cong \operatorname{Spec}(\mathbb{C}[x, y, z])$ be a hypersurface $f(x, y, z)=0$ such that the origin $O \in \mathbb{C}^{3}$ is a non-smooth isolated singular point of $X \subset \mathbb{C}^{3}$, and let

$$
f(x, y, z)=f_{d}(x, y, z)+f_{d+1}(x, y, z)+\ldots,
$$


where $f_{i}(x, y, z)$ is a quasi-homogeneous polynomial of quasi-homogeneous degree $i \geq 2$ with respect to positive integer weights $\operatorname{wt}(x)=a, \operatorname{wt}(y)=b, \operatorname{wt}(z)=c$. Suppose that the origin $O \in \mathbb{C}^{3}$ is an isolated singular point of the hypersurface $f_{d}(x, y, z)=0,2 a \leq d$, $2 b \leq d, 2 c \leq d$ and $a+b+c>d$. Then

- $(a, b, c)=(n+1, n+1,2)$ implies $O \in X$ is a singularity of type $\mathbb{A}_{n}$;

- $(a, b, c)=(n-1, n-2,2)$ implies $O \in X$ is a singularity of type $\mathbb{D}_{n}$;

- $(a, b, c)=(6,4,3)$ implies $O \in X$ is a singularity of type $\mathbb{E}_{6}$;

- $(a, b, c)=(9,6,4)$ implies $O \in X$ is a singularity of type $\mathbb{E}_{7}$;

- $(a, b, c)=(15,10,6)$ implies $O \in X$ is a singularity of type $\mathbb{E}_{8}$.

The following result is due to F. Enriques (see [36, [58, [28, 61]).

Theorem 2.11. Let $X \subset \mathbb{P}^{n}$ be a variety of degree $n-\operatorname{dim}(X)+1$ such that $X$ is not contained in any hyperplane. Then $X$ is one of the following:

- a projective space $\mathbb{P}^{n}$;

- a quadric hypersurface in $\mathbb{P}^{n}$;

- an image of a rational scroll $\mathbb{F}\left(d_{1}, \ldots, d_{k}\right)=\operatorname{Proj}\left(\oplus_{i=1}^{k} \mathcal{O}_{\mathbb{P}^{1}}\left(d_{i}\right)\right)$ via the map given by the tautological line bundle, where $0 \neq d_{1} \geq \ldots d_{k} \geq 0$ and $n+1=\sum_{i=1}^{k}\left(d_{i}+1\right)$;

- a Veronese surface in $\mathbb{P}^{5}$ when $n=5$;

- a cone in $\mathbb{P}^{n}$ over a Veronese surface in $\mathbb{P}^{5}$.

It is easy to see that the varieties in Theorem 2.11 have the smallest possible degree among all varieties of the same dimension in $\mathbb{P}^{n}$.

The vanishing theorem of Kawamata-Viehweg (see [70, [119]) and elementary properties of linear systems on K3 surfaces (see [104]) imply the following well known result (see [58, 61, 68]).

Theorem 2.12. Let $X$ be a Fano 3-fold with canonical Gorenstein singularities, such that the linear system $\left|-K_{X}\right|$ has no base points but the anticanonical divisor $-K_{X}$ is not very ample. Then $\phi_{\left|-K_{X}\right|}: X \rightarrow V \subset \mathbb{P}^{n}$ is a double cover and $V \subset \mathbb{P}^{n}$ is a subvariety of minimal degree, i.e. $\operatorname{deg}(V)=n-2$, where $n=-\frac{1}{2} K_{X}^{3}+2$.

The following result is a theorem of Noether-Enriques-Petri (see [107, [41]).

Theorem 2.13. Let $C \subset \mathbb{P}^{g-1}$ be a canonically embedded smooth irreducible curve whose genus $g(C)$ is at least 3 . Then the following holds:

- the curve $C \subset \mathbb{P}^{g-1}$ is projectively normal;

- in the case $g(C)=3$ the curve $C$ is a plane quartic curve;

- in the case $g(C) \geq 4$ the graded ideal $I_{C}$ of the curve $C \subset \mathbb{P}^{g-1}$ is generated by the components of degree 2 and 3 , i.e. the curve $C \subset \mathbb{P}^{g-1}$ is cut by quadrics and cubics in $\mathbb{P}^{g-1}$ in scheme-theoretic sense;

- in the case $g(C) \geq 4$ the graded ideal $I_{C}$ of the curve $C \subset \mathbb{P}^{g-1}$ is generated by the components of degree 2 except the following two cases:

- the curve $C$ is trigonal, i.e. there is a map $\psi: C \rightarrow \mathbb{P}^{1}$ of degree 3 ;

- the curve $C$ is a plane quintic curve, i.e. $g(C)=6$;

- in the trigonal case the quadrics in $\mathbb{P}^{g-1}$ passing through the curve $C$ cut out either a possibly singular irreducible quadric surface when $g(C)=4$ or a smooth irreducible surface of degree $g-2$ which is an image of $\operatorname{Proj}\left(\mathcal{O}_{\mathbb{P}^{1}}\left(d_{1}\right) \oplus \mathcal{O}_{\mathbb{P}^{1}}\left(d_{2}\right)\right)$ via the map given by the tautological line bundle, where $d_{1} \geq d_{2}>0$ and $g=d_{1}+d_{2}+2$;

- in the case when $C$ is a plane quintic curve the quadrics in $\mathbb{P}^{5}$ passing through the curve $C$ cut out a Veronese surface. 
The following result is implied by Theorem [2.13 (see [58, 61, 68]).

Theorem 2.14. Let $X \subset \mathbb{P}^{n}$ be an anticanonically embedded Fano 3-fold with canonical singularities, i.e. $-\left.K_{X} \sim \mathcal{O}_{\mathbb{P}^{n}}(1)\right|_{X}$ and $n=-\frac{1}{2} K_{X}^{3}+2$. Then the following holds:

- the 3-fold $X$ is projectively normal in $\mathbb{P}^{n}$;

- in the case $-K_{X}^{3}=4$ the 3-fold $X$ is a quartic 3-fold in $\mathbb{P}^{4}$;

- in the case $-K_{X}^{3} \geq 6$ the graded ideal $I_{X}$ of the 3-fold $X \subset \mathbb{P}^{n}$ is generated by the components of degree 2 and 3 ;

- in the case $-K_{X}^{3} \geq 6$ the graded ideal $I_{X}$ of the 3-fold $X \subset \mathbb{P}^{n}$ is generated by the components of degree 2 except the following case:

- for a general linear subspace $\Pi \subset \mathbb{P}^{n}$ of codimension 2 the curve $X \cap \Pi$ is either a canonically embedded smooth trigonal curve or a canonically embedded smooth plane quintic curve and $\operatorname{deg}\left(X \subset \mathbb{P}^{n}\right)=10$;

- in the trigonal case the quadrics in $\mathbb{P}^{n}$ passing through $X$ cut out either a possibly singular irreducible quadric 4-fold when $-K_{X}^{3}=6$ or a 4-fold of degree $n-3$ which is an image of a rational scroll $\operatorname{Proj}\left(\oplus_{i=1}^{4} \mathcal{O}_{\mathbb{P}^{1}}\left(d_{i}\right)\right)$ via the map given by the tautological line bundle, where $0 \neq d_{1} \geq \ldots \geq d_{4} \geq 0$ and $n+1=\sum_{i=1}^{4}\left(d_{i}+1\right)$;

- in the case when $X \cap \Pi$ is a canonically embedded plane quintic curve the quadrics in $\mathbb{P}^{7}$ passing through $X$ cut out a 4-fold cone over a Veronese surface.

The following result is Claim 6.9 in 94 .

Proposition 2.15. Let $X$ be a 3-fold with $\mathrm{cDV}$ singularities, $\Gamma \subset \operatorname{Sing}(X)$ be a smooth curve considered as a reduced subscheme of the 3-fold $X$, and $f: V \rightarrow X$ be a blow up of the curve $\Gamma$. Then $V$ has at most cDV singularities and $K_{V} \sim f^{*}\left(K_{X}\right)$, i.e. $f$ is crepant.

The following result is proved in [110] and is a special case of a conjectural rationality criterion of a standard three-dimensional conic bundle (see [60, 62, 63, 64]).

Theorem 2.16. Let $Y$ be a smooth 3-fold, $Z$ be either $\mathbb{P}^{2}$ or a minimal rational ruled surface $\mathbb{F}_{r}$, and $\xi: Y \rightarrow Z$ be a conic bundle with $\operatorname{Pic}(Y / Z)=\mathbb{Z}$ such that $\left|2 K_{Z}+\Delta\right| \neq \emptyset$, where $\Delta \subset Z$ is a degeneration divisor of $\xi: Y \rightarrow Z$. Then $Y$ is non-rational.

Remark 2.17. In the notations of Theorem 2.16 the condition $\left|2 K_{Z}+\Delta\right|=\emptyset$ implies the rationality of the 3 -fold $Y$ except the case when the commutative diagram

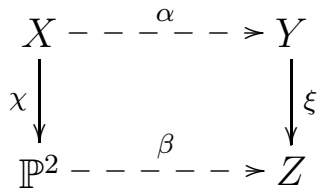

exists, where $\alpha$ and $\beta$ are birational maps, the 3 -fold $X$ is smooth, $\chi: X \rightarrow \mathbb{P}^{2}$ is a conic bundle with $\operatorname{Pic}\left(X / \mathbb{P}^{2}\right)=\mathbb{Z}$ whose degeneration divisor $D \subset \mathbb{P}^{2}$ is a quintic curve and the double cover $\psi: \tilde{D} \rightarrow D$ induced by $\chi$ corresponds to an even $\theta$-characteristic (see [63]).

The following result of [85] is a special case of a more general result in [75] (see also [74, [78]), which generalizes the standard degeneration technique (see 66]).

Theorem 2.18. Let $\xi: Y \rightarrow Z$ be a flat proper morphism with irreducible and reduced geometric fibers. Then there are countably many closed subvarieties $Z_{i} \subset Z$ such that for an arbitrary closed point $s \in Z$ the fiber $\xi^{-1}(s)$ is ruled if and only if $s \in \cup Z_{i}$.

The following claim is due to [102]. 
Proposition 2.19. Let $V$ be a rational scroll $\operatorname{Proj}\left(\sum_{i=1}^{k} \mathcal{O}_{\mathbb{P}^{1}}\left(d_{i}\right)\right)$ and $f: V \rightarrow \mathbb{P}^{1}$ be a natural projection. Then $\operatorname{Pic}(V) \cong \mathbb{Z} M \oplus \mathbb{Z} L$, where $M$ is a tautological line bundle on the variety $V$ and $L$ is a fiber of the projection $f$. Let $\left(t_{1}: t_{2}\right)$ be homogeneous coordinates on the base $\mathbb{P}^{1}$ and $\left(x_{1}: \ldots: x_{k}\right)$ be homogeneous coordinates on the fiber of $f$, which is isomorphic to $\mathbb{P}^{k-1}$. This coordinates coincide with coordinates on $\mathcal{O}_{\mathbb{P}^{1}}\left(d_{i}\right)$. Then $|a M+b L|$ is generated by bihomogeneous polynomials

$$
c_{i_{1}, \ldots, i_{k}} x_{1}^{i_{1}} x_{2}^{i_{2}} \ldots x_{k}^{i_{k}}
$$

where $\sum_{j=1}^{k} i_{j}=a, i_{j} \geqslant 0$ and $c_{i_{1}, \ldots, i_{k}}=c_{i_{1}, \ldots, i_{k}}\left(t_{0}: t_{2}\right)$ is a homogeneous polynomial of degree $b+\sum_{j=1}^{k} i_{j} d_{j}$.

For details see [102, Chapter 2.

Actually, Proposition 2.19 implies the following result known as a lemma of M. Reid.

Corollary 2.20. Let $V$ be a rational scroll $\operatorname{Proj}\left(\sum_{i=1}^{k} \mathcal{O}_{\mathbb{P}^{1}}\left(d_{i}\right)\right)$ with $d_{1} \geq \ldots \geq d_{k} \geq 0$, and let $Y_{j} \subset V$ be a "negative" rational subscroll Proj $\left(\oplus_{i=j}^{k} \mathcal{O}_{\mathbb{P}^{1}}\left(d_{i}\right)\right)$ corresponding to a natural projection $\oplus_{i=1}^{k} \mathcal{O}_{\mathbb{P}^{1}}\left(d_{i}\right) \rightarrow \oplus_{i=j}^{k} \mathcal{O}_{\mathbb{P}^{1}}\left(d_{i}\right)$. Take an effective divisor $D \subset V$ that is rationally equivalent to $a M+b L$, where $M$ is tautological line bundle on $V, L$ is a fiber of the natural projection to $\mathbb{P}^{1}$, and $a$ and $b$ are integers. Then mult $_{Y_{j}}(D) \geq q$ for $q \in \mathbb{N}$ if and only if the inequality $a d_{j}+b+\left(d_{1}-d_{j}\right)(q-1)<0$ holds.

The following result is implied by the Riemann-Roch theorem (see [58, 61, 68]), the vanishing theorem of Kawamata-Viehweg (see [70], [119]), the rationality of canonical singularities (see [72, [76]) and the global-to-local spectral sequence.

Proposition 2.21. Let $X$ be a Fano 3-fold with canonical Gorenstein singularities. Then $h^{0}\left(\mathcal{O}_{X}\left(-m K_{X}\right)\right)=\frac{m(m+1)(2 m+1)}{12}\left(-K_{X}\right)^{3}+2 m+1$.

The following two results are due to [51, [80, [81, [52, [53], [54, [57]. For their modern proof see [87] and 65].

Theorem 2.22. Let $W$ be a smooth minimal geometrically irreducible and geometrically rational surface defined over a perfect field $\mathbb{F}$, i.e. there is no curve on $W$ that can be contracted to a smooth point over $\mathbb{F}$ and the surface $W$ is rational and irreducible over $\overline{\mathbb{F}}$. Then either $\operatorname{Pic}(W) \cong \mathbb{Z}$ and $W$ is a smooth del Pezzo surface or $\operatorname{Pic}(W) \cong \mathbb{Z} \oplus \mathbb{Z}$ and there is a conic bundle $\pi: W \rightarrow Z$.

Theorem 2.23. Let $W$ be a smooth minimal geometrically irreducible and geometrically rational surface defined over a perfect field $\mathbb{F}$. Then the surface $W$ is rational over $\mathbb{F}$ if and only if the surface $W$ has an $\mathbb{F}$-point and the inequality $K_{W}^{2} \geq 5$ holds.

The following result is due to 80 .

Theorem 2.24. Let $W$ be a smooth geometrically irreducible and geometrically rational surface defined over a $\mathrm{C}_{1}$-field $\mathbb{F}$, e.g. $\mathbb{F}=\mathbb{C}(x)$. Then the surface $W$ has an $\mathbb{F}$-point.

The following result is due to [75].

Theorem 2.25. Let $Y$ be a projective variety and $g: Y \rightarrow R$ be a morphism with a section onto a smooth curve $R$. Suppose that we have a set of closed points $\left\{r_{1}, \ldots, r_{k}\right\} \in R$ such that each fiber $Y_{i}=g^{-1}\left(r_{i}\right)$ is smooth and separably rationally connected. Then for a set of closed points $y_{i} \in Y_{i}$ there is a section $C \subset Y$ of the morphism $g$ passing through each point $y_{i}$. 


\section{Hyperelliptic FAno 3-Folds.}

In this section we will prove Theorem 1.5. Let $X$ be a Fano variety with canonical Gorenstein singularities, such that the linear system $\left|-K_{X}\right|$ has no base points, but the induced morphism $\phi_{\left|-K_{X}\right|}$ is not an embedding. Then $\phi_{\left|-K_{X}\right|}: X \rightarrow Y \subset \mathbb{P}^{n}$ is a double cover and $\operatorname{deg}\left(Y \subset \mathbb{P}^{n}\right)=n-2$, where $n=-\frac{1}{2} K_{X}^{3}+2$.

Remark 3.1. In the case $-K_{X}^{3}=2$ the 3 -fold $Y$ is nothing but $\mathbb{P}^{3}$ and $\phi_{\left|-K_{X}\right|}$ is a double cover ramified in a possibly singular sextic surface. In this case $X$ can be considered as a hypersurface of degree 6 in $\mathbb{P}\left(1^{4}, 3\right)$. The birational geometry of such $X$ was studied in [8], 118, 59, 95, [98, 44, [15], 18].

Remark 3.2. In the case $-K_{X}^{3}=4$ the 3 -fold $Y$ is a possibly singular quadric in $\mathbb{P}^{4}$ and $\phi_{\left|-K_{X}\right|}$ is a double cover branched over a surface that is cut on $Y$ by a quartic hypersurface in $\mathbb{P}^{4}$. In this case $X$ can be considered as a complete intersection of a quadric cone and a quartic in $\mathbb{P}\left(1^{5}, 2\right)$. The birational geometry of such $X$ was studied in [8], 118, [59, [95], 42], 43].

Thus, we may assume that $-K_{X}^{3} \geq 6$. Hence, Theorem 2.11 implies that either $-K_{X}^{3}=$ 8 and $Y \subset \mathbb{P}^{6}$ is a cone over a Veronese surface $F_{4} \subset \mathbb{P}^{5}$ or $Y$ is image of a rational scroll $\mathbb{F}\left(d_{1}, d_{2}, d_{3}\right)=\operatorname{Proj}\left(\oplus_{i=1}^{3} \mathcal{O}_{\mathbb{P}^{1}}\left(d_{i}\right)\right)$ via the map given by the tautological line bundle, where $0 \neq d_{1} \geq \ldots d_{k} \geq 0$ and $-K_{X}^{3}=2\left(d_{1}+d_{2}+d_{3}\right)$.

Lemma 3.3. Suppose that $Y$ is a cone over a Veronese surface $F_{4}$ with a vertex $O$. Then $X$ is a hypersurface of degree 6 in $\mathbb{P}\left(1^{3}, 2,3\right)$.

Proof. We have $Y \cong \mathbb{P}\left(1^{3}, 2\right)$. The double cover $\phi_{\left|-K_{X}\right|}$ is branched over the vertex $O$, because $O$ is not a Gorenstein point on $Y$. On the other hand, the equality $-K_{X}^{3}=8$ implies that the double cover $\phi_{\left|-K_{X}\right|}$ is branched over a divisor $D \subset Y$ such that $D \sim$ $\mathcal{O}_{Y}(6)$. Like in the smooth case (see [58]) the latter implies (cf. [105]) that $X$ is a hypersurface of degree 6 in $\mathbb{P}\left(1^{3}, 2,3\right)$.

Therefore, we may assume that there is a birational morphism $f: U \rightarrow Y$ for $U=$ $\operatorname{Proj}\left(\oplus_{i=1}^{3} \mathcal{O}_{\mathbb{P}^{1}}\left(d_{i}\right)\right)$ and $f=\phi_{|M|}$, where $M$ is a tautological line bundle on $U, 0 \neq d_{1} \geq$ $\ldots d_{k} \geq 0$ and $-K_{X}^{3}=2\left(d_{1}+d_{2}+d_{3}\right) \geq 6$.

Lemma 3.4. Suppose that $d_{2}=d_{3}=0$. Then $X$ is either a hypersurface in $\mathbb{P}\left(1^{2}, 3^{2}, 5\right)$ of degree 10 or a hypersurface in $\mathbb{P}\left(1^{2}, 4^{2}, 6\right)$ of degree 12 .

Proof. Take a sufficiently general divisor $H \in\left|-K_{X}\right|$. Then $H$ is a K3 surface with Du Val singularities and $f(H)$ is a cone in $\mathbb{P}^{n-1}$ over a rational normal curve. Moreover, the restriction map

$$
H^{0}\left(\mathcal{O}_{X}\left(-K_{X}\right)\right) \rightarrow H^{0}\left(\mathcal{O}_{H}\left(-\left.K_{X}\right|_{H}\right)\right)
$$

is surjective due to the vanishing of $H^{1}\left(\mathcal{O}_{X}\right)$. Hence, the equalities $d_{1}=d_{2}=0$ imply $-K_{X}^{3} \leq 8$ by [104].

Thus, we have two possible cases $d_{1}=3$ and $d_{1}=4$. In the former case $Y \cong \mathbb{P}\left(1^{2}, 3^{2}\right)$, in the latter case $Y \cong \mathbb{P}\left(1^{2}, 4^{2}\right)$. Now we may proceed as in the proof of Lemma 3.3 to prove the required claim.

Remark 3.5. It is possible to show explicitly the existence of a hypersurface in $\mathbb{P}\left(1^{2}, 3^{2}, 5\right)$ of degree 10 and a hypersurface in $\mathbb{P}\left(1^{2}, 4^{2}, 6\right)$ of degree 12 having canonical Gorenstein singularities using the basic properties of a hypersurface in a weighted projective spaces (see [35]). However, we will prove it in a different and more geometric way together with other possible cases. 
Let $V$ be a normalization of a fibered product $X \times_{Y} U, \pi: V \rightarrow U$ be a double cover induced by $\phi_{\left|-K_{X}\right|}: X \rightarrow Y$, and $h: V \rightarrow X$ be a birational morphism induced by $f: U \rightarrow Y$.

Lemma 3.6. The 3-fold $V$ has canonical Gorenstein singularities, the anticanonical divisor $-K_{V}$ is nef and big, and $K_{V} \sim h^{*}\left(K_{X}\right)$, i.e. the map $h$ is crepant.

Proof. In the case when $d_{2} \neq 0$ the 3 -folds $X$ and $V$ are isomorphic in codimension 2 . The latter easily implies the claim (cf. [71]).

Thus, we may assume $d_{2}=0$. Then $f: U \rightarrow Y$ contracts a divisor $D \subset U$ to a curve $C \cong \mathbb{P}^{1}$ and Lemma 3.4 implies that either $d_{1}=3$ or $d_{1}=4$. In both cases $\phi_{\left|-K_{X}\right|}$ must be ramified in the curve $C$, because $Y$ is non-Gorenstein at the general point of the curve $C$.

Let $R \subset U$ be a ramification divisor of $\pi: V \rightarrow U, M$ be a tautological line bundle on $U$ and $L$ be a fiber of the natural projection of $U$ to $\mathbb{P}^{1}$. Then the equivalences

$$
-K_{X} \sim \phi_{\left|-K_{X}\right|}^{*}\left(\left.\mathcal{O}_{P}^{n}(1)\right|_{Y}\right)
$$

and $M \sim f^{*}\left(\left.\mathcal{O}_{P}^{n}(1)\right|_{Y}\right)$ imply $R \sim 4 M-2\left(d_{1}-2\right) L+a D$ for $a \in \mathbb{Z}$. Moreover, the canonicity of $X$ implies $a \geq 0$. However, the inequality $a>0$ together with Corollary 2.20 imply $R=2 D \cup S$, where $S$ is an effective divisor on $U$, because $D \sim M-d_{1} L$. The latter contradicts the normality of $V$. Thus, $R \sim 4 M-2\left(d_{1}-2\right) L$ and $-K_{V} \sim f^{*}(M) \sim h^{*}\left(K_{X}\right)$, which easily implies the claim.

Let $D \subset U$ be a ramification divisor of $\pi: V \rightarrow U, M$ be a tautological line bundle on $U$ and $L$ be a fiber of the natural projection of $U$ to $\mathbb{P}^{1}$. Then $-K_{V} \sim \pi^{*}(M)$ by construction. Hence, $D \sim 4 M-2\left(d_{1}+d_{2}+d_{3}-2\right) L$. Let $Y_{2} \subset V$ and $Y_{3} \subset V$ be subscrolls corresponding to natural projections $\oplus_{i=1}^{3} \mathcal{O}_{\mathbb{P}^{1}}\left(d_{i}\right) \rightarrow \mathcal{O}_{\mathbb{P}^{1}}\left(d_{2}\right) \oplus \mathcal{O}_{\mathbb{P}^{1}}\left(d_{3}\right)$ and $\oplus_{i=1}^{3} \mathcal{O}_{\mathbb{P}^{1}}\left(d_{i}\right) \rightarrow \mathcal{O}_{\mathbb{P}^{1}}\left(d_{3}\right)$ respectively. Then $Y_{2} \cong \operatorname{Proj}\left(\mathcal{O}_{\mathbb{P}^{1}}\left(d_{2}\right) \oplus \mathcal{O}_{\mathbb{P}^{1}}\left(d_{3}\right)\right)$ and $Y_{3} \cong \mathbb{P}^{1}$.

Lemma 3.7. The inequalities mult $_{Y_{2}}(D) \leq 1$ and mult $_{Y_{3}}(D) \leq 3$ hold.

Proof. The normality of $V$ implies $\operatorname{mult}_{Y_{2}}(D) \leq 1$. Let $d=\operatorname{mult}_{Y_{3}}(D) \geq 2$. Then the local equation of the 3 -fold $V$ in the neighbourhood of the general point of the curve $C=\pi^{-1}\left(Y_{2}\right)$ is

$$
\omega^{2}=f_{d}(x, y)+f_{d+1}(x, y)+\ldots \subset \operatorname{Spec}(\mathbb{C}[x, y, z, \omega]),
$$

where $x=y=0$ are local equations of the curve $C$ and $f_{i}(x, y)$ is a homogeneous polynomial of degree $i$. On the other hand, the singularities of $V$ at the general point of the curve $C$ locally must be isomorphic to one of the following types of singularities: $\mathbb{C} \times \mathbb{A}_{n}, \mathbb{C} \times \mathbb{D}_{n}, \mathbb{C} \times \mathbb{E}_{6}, \mathbb{C} \times \mathbb{E}_{7}, \mathbb{C} \times \mathbb{E}_{8}$. Now, Theorem 2.9] implies $d \leq 3$.

Therefore, Corollary 2.20 implies the inequalities $d_{1}-d_{3}-2 d_{2}+4 \geq 0, d_{2}-d_{1}-2 d_{3}+4 \geq$ 0 . Moreover, we also have $0 \neq d_{1} \geq \ldots \geq d_{3} \geq 0$ and $d_{1}+d_{2}+d_{3} \geq 3$ by assumption. The obtained inequalities give exactly 44 different rational scrolls $\mathbb{F}\left(d_{1}, d_{2}, d_{3}\right)=$ $\operatorname{Proj}\left(\oplus_{i=1}^{3} \mathcal{O}_{\mathbb{P}^{1}}\left(d_{i}\right)\right)$ with a ramification divisor $D \sim 4 M-2\left(d_{1}+d_{2}+d_{3}-2\right) L$, where $M$ is a tautological line bundle on $\mathbb{F}\left(d_{1}, d_{2}, d_{3}\right)$ and $L$ is a fiber of the natural projection to $\mathbb{P}^{1}$.

Remark 3.8. The 3-fold $X$ is an anticanonical model of the 3-fold $V$, i.e. $X \cong \phi_{\left|-r K_{V}\right|}(V)$ for $r \gg 0$. Thus, the contraction theorem (see [72]) implies that the 3-fold $X$ is a Fano 3 -fold with canonical Gorenstein singularities if and only if $V$ has canonical Gorenstein singularities and $-K_{V}$ is nef and big. On the other hand, the 3 -fold $V$ is uniquely 
determined by a rational scroll $\mathbb{F}\left(d_{1}, d_{2}, d_{3}\right)$ with a ramification divisor $D \in \mid 4 M-2\left(d_{1}+\right.$ $\left.d_{2}+d_{3}-2\right) L \mid$. The only proble is that a priori we may fail to find a divisor $D \in$ $\left|4 M-2\left(d_{1}+d_{2}+d_{3}-2\right) L\right|$ such that the corresponding double cover $V$ has a canonical singularities.

In the rest of the section we will explicitly show that in every obtained case there is a divisor $D \in\left|4 M-2\left(d_{1}+d_{2}+d_{3}-2\right) L\right|$, such that $V$ has canonical singularities. We will use Corollary 2.7 together with Proposition 2.19] to prove the existence of the divisor $D$ in every possible case. The latter will conclude the proof of Theorem 1.5.

Remark 3.9. Actually, the same idea was used during the classification of smooth hyperelliptic Fano 3-folds (see [58, 61]). However, the obtained inequality are much stronger in the smooth case and the calculations are much shorter. The same method was used in 14 in the effective boundness of the hyperelliptic Fano 3-folds with canonical Gorenstein singularities, but there is a gap in the proof of Lemma 3.2 in 14. Namely, the stronger inequality mult $_{Y_{3}}(D) \leq 2$ was used instead of the inequality mult $Y_{Y_{3}}(D) \leq 3$. The latter gave a wrong bound $-K_{X}^{3} \leq 16$ instead of the right one $-K_{X}^{3} \leq 40$, which is sharp a posteriori. However, the bound $-K_{X}^{3} \leq 40$ still can be used to get the main result of [14], which is obsolete now due to 94 .

First, let us consider one possible case in full details.

Example 3.10. Let $\pi: V \rightarrow \mathbb{F}(6,2,0)$ be a double cover of the scroll branched over a sufficiently general divisor $D \subset \mathbb{F}(6,2,0)$ such that $D \sim 4 M-12 L$, where $M$ is a class of a tautological line bundle on $\mathbb{F}(6,2,0)$ and $L$ is a class of the fiber of the projection of $\mathbb{F}(6,2,0)$ to $\mathbb{P}^{1}$. We must show that the 3 -fold $V$ has canonical singularities.

By Proposition 2.19 the divisor $D$ is given by the zeroes of the bihomogeneous polynomial

$$
\begin{aligned}
\alpha_{12}\left(t_{1}, t_{2}\right) x_{1}^{4}+\alpha_{8}\left(t_{1}, t_{2}\right) x_{1}^{3} x_{2}+\alpha_{6}\left(t_{1}, t_{2}\right) x_{1}^{3} x_{3}+\alpha_{4}\left(t_{1}, t_{2}\right) x_{1}^{2} x_{2}^{2}+ \\
\alpha_{2}\left(t_{1}, t_{2}\right) x_{1}^{2} x_{2} x_{3}+\alpha_{0}^{1}\left(t_{1}, t_{2}\right) x_{1}^{2} x_{3}^{2}+\alpha_{0}^{2}\left(t_{1}, t_{2}\right) x_{1} x_{2}^{3},
\end{aligned}
$$

where $\alpha_{d}\left(t_{1}, t_{2}\right)$ (or $\left.\alpha_{0}^{i}\left(t_{1}, t_{2}\right)\right)$ is an arbitrary form of degree $d$. Let $E \subset \mathbb{F}(6,2,0)$ be a surface given by $x_{1}=0$ and $C \subset \mathbb{F}(6,2,0)$ be a curve given by $x_{1}=x_{2}=0$. Note that the base locus of $|4 M-12 L|$ is exactly $E$ (in particular, $D \backslash E$ and $V \backslash \pi^{-1}(E)$ are smooth by Bertini theorem). As the automorphism group of $E \cong \mathbb{F}(4,0)$ acts transitively on $E \backslash C$ and $D$ has multiplicity 1 at a general point of $E$, by Corollary 2.7 it is sufficient to prove that for any point $p$ of $C$ there exists a divisor $D$ such that the corresponding variety $V$ has a canonical singularity at a general point of $\pi^{-1}(C)$.

Let $Y$ be a fiber of the projection of $\mathbb{F}(6,2,0)$ to $\mathbb{P}^{1}$ over a sufficiently general point $P \in \mathbb{P}^{1}$ and $Z=\pi^{-1}(Y)$. Then $Z$ is a del Pezzo surface of degree 2. Moreover, the only possible singular point of $Z$ is a point $O=\pi^{-1}(C \cap Y)$. Let us prove that $O$ is a Du Val point on $Z$. The latter implies the canonicity of the singularities of $V$ by Corollary 2.7.

Let the homogeneous coordinates of the point $P \in \mathbb{P}^{1}$ be $(\gamma: \delta)$. Then the del Pezzo surface $Z$ can be given as a hypersurface

$$
\omega^{2}=\alpha_{12} x_{1}^{4}+\alpha_{8} x_{1}^{3} x_{2}+\alpha_{6} x_{1}^{3} x_{3}+\alpha_{4} x_{1}^{2} x_{2}^{2}+\alpha_{2} x_{1}^{2} x_{2} x_{3}+\alpha_{0}^{1} x_{1}^{2} x_{3}^{2}+\alpha_{0}^{2} x_{1} x_{2}^{3}
$$

in $\mathbb{P}(1,1,1,2) \cong \operatorname{Proj}\left(\mathbb{C}\left[x_{1}, x_{2}, x_{3}, \omega\right]\right)$ where $\alpha_{d}^{(i)}=\alpha_{d}(\gamma, \delta)$. The generality of $Y$ implies $\alpha_{i} \neq 0$ for all $i$. Therefore, for convenience we may put $\alpha_{i}^{j}=1$ for all $i$ and $j$. 
Let $\omega=x, x_{1}=y, x_{2}=z$ and $x_{3}=1$. Then the local equation of $Z$ is

$$
x^{2}+y^{4}+y^{3} z+y^{3}+y^{2} z^{2}+y^{2} z+y^{2}+y z^{3}=0
$$

in the neighbourhood of the point $O$. Let $\operatorname{wt}(x)=3, \operatorname{wt}(y)=3$ and $\operatorname{wt}(z)=1$. Then $\operatorname{wt}\left(x^{2}+y^{2}+y z^{3}\right)=6$ and $\operatorname{wt}\left(y^{4}\right)=12, \operatorname{wt}\left(y^{3} z\right)=10, \operatorname{wt}\left(y^{3}\right)=9, \operatorname{wt}\left(y^{2} z^{2}\right)=8$ and $\operatorname{wt}\left(y^{2} z\right)=7$. Moreover, the singularity given by the equation $x^{2}+y^{2}+y z^{3}=0$ is isolated. Therefore, the singularity at the point $O$ on the surface $Z$ is locally isomorphic to a Du Val singularity of type $\mathbb{A}_{5}$ by Theorem 2.10. In particular, at a generic point of the curve $\pi^{-1}(C)$ the 3 -fold $V$ has singularity of type $\mathbb{A}_{5} \times \mathbb{C}$.

Actually, we may assume that the point $P \in \mathbb{P}^{1}$ is not just a general point but an arbitrary point due to generality in the choice of $D$, i.e. for any $P \in \mathbb{P}^{1}$ we can take such homogeneous polynomials $\alpha_{i}$ that $\alpha_{i}(P) \neq 0$ and repeat all the previous arguments in the neighbourhood of the corresponding point $O=\pi^{-1}(C \cap Y)$. Hence, the singularities of $V$ are canonical by Corollary 2.7

In the rest of the section we will consider all the possible cases following the pattern in Example 3.10 but omitting the unnecessary details. We will label every case by the corresponding name of $X$ used in the statement of Theorem 1.5, i.e. $H_{4}, H_{5}, \ldots, H_{47}$. In the proof of every possible case we will use the notations of Example 3.10 for simplicity.

$\left(H_{4}\right)$ : A double cover $V \longrightarrow \mathbb{F}(1,1,1)$. The ramification divisor $D$ lies in the base point free linear system $|4 M-2 L|$ and is smooth. Hence the singularities of $V$ are canonical.

$\left(H_{5}\right)$ : A double cover $V \longrightarrow \mathbb{F}(2,1,0)$. The ramification divisor $D$ lies in the linear system $|4 M-2 L|$ and is given by the form

$$
\begin{aligned}
\alpha_{6} x_{1}^{4}+ & \alpha_{5} x_{1}^{3} x_{2}+\alpha_{4}^{1} x_{1}^{3} x_{3}+\alpha_{4}^{2} x_{1}^{2} x_{2}^{2}+\alpha_{3}^{1} x_{1}^{2} x_{2} x_{3}+\alpha_{2}^{1} x_{1}^{2} x_{3}^{2}+ \\
& +\alpha_{3}^{2} x_{1} x_{2}^{3}+\alpha_{2}^{2} x_{1} x_{2}^{2} x_{3}+\alpha_{1}^{1} x_{1} x_{2} x_{3}^{2}+\alpha_{0}^{1} x_{1} x_{3}^{3}+\alpha_{2}^{3} x_{2}^{4}+\alpha_{1}^{2} x_{2}^{3} x_{3}+\alpha_{0}^{2} x_{2}^{2} x_{3}^{2} .
\end{aligned}
$$

The curve $C$ given by $x_{1}=x_{2}=0$ is the only base curve of this linear system. As in Example 3.10, on the inverse image of the fiber $Y$ in the neighbourhood of the point $O=C \cap Y$ after a coordinate change $\omega=x, x_{1}=y, x_{2}=z$ and $x_{3}=1$ we have a (possible) singularity at $O$ given by the zeroes of the polynomial

$$
x^{2}+y^{3} z+y^{3}+y^{2} z^{2}+y^{2} z+y^{2}+y z^{3}+y z^{2}+y z+y+z^{4}+z^{3}+z^{2},
$$

and so $O$ is in fact a smooth point. Hence by Corollary 2.7 the singularities of $V$ are canonical.

$\left(H_{6}\right)$ : A double cover $V \longrightarrow \mathbb{F}(2,1,1)$. The ramification divisor $D$ lies in the base point free linear system $|4 M-4 L|$ and is smooth. Hence the singularities of $V$ are canonical.

$\left(H_{7}\right)$ : A double cover $V \longrightarrow \mathbb{F}(2,2,0)$. The ramification divisor $D$ lies in the linear system $|4 M-4 L|$ and is given by the form

$$
\begin{aligned}
\alpha_{4}^{1} x_{1}^{4}+\alpha_{4}^{2} x_{1}^{3} x_{2}+\alpha_{4}^{3} x_{1}^{2} x_{2}^{2} & +\alpha_{4}^{4} x_{1} x_{2}^{3}+\alpha_{4}^{5} x_{2}^{4}+\alpha_{2}^{1} x_{1}^{3} x_{3}+\alpha_{2}^{2} x_{1}^{2} x_{2} x_{3}+ \\
& +\alpha_{2}^{3} x_{1} x_{2}^{2} x_{3}+\alpha_{2}^{4} x_{2}^{3} x_{3}+\alpha_{0}^{1} x_{1}^{2} x_{3}^{2}+\alpha_{0}^{2} x_{1} x_{2} x_{3}^{2}+\alpha_{0}^{3} x_{2}^{2} x_{3}^{2} .
\end{aligned}
$$

The curve $C$ given by $x_{1}=x_{2}=0$ is the only base curve of this linear system. As in Example 3.10, on the inverse image of the fiber $Y$ in the neighbourhood of the point $O=C \cap Y$ after a coordinate change $\omega=x, x_{1}=y, x_{2}=z$ and $x_{3}=1$ we have a singularity at $O$ given by the zeroes of the polynomial

$$
x^{2}+P_{2}(y, z)+P_{3}(y, z)+P_{4}(y, z),
$$


where $P_{i}$ is a homogeneous polynomial of degree $i$. If we put $\operatorname{wt}(x)=1, \operatorname{wt}(y)=1$ and $\operatorname{wt}(z)=1$, it's easy to check that the singularity given by the term of the lowest weight is isolated and Du Val. The weights coincide with the weights of $\mathbb{A}_{1}$. Then by Theorem 2.10 in the neighbourhood of $O$ the singularity is isomorphic to $\mathbb{A}_{1} \times \mathbb{C}$, and by Corollary 2.7 the singularities of $V$ are canonical.

$\left(H_{8}\right)$ : A double cover $V \longrightarrow \mathbb{F}(2,2,1)$. The ramification divisor $D$ lies in the linear system $|4 M-6 L|$ and is given by the form

$$
\begin{aligned}
\alpha_{2}^{1} x_{1}^{4}+\alpha_{2}^{2} x_{1}^{3} x_{2}+\alpha_{2}^{3} x_{1}^{2} x_{2}^{2}+\alpha_{4}^{2} x_{1} x_{2}^{3}+\alpha_{2}^{5} x_{2}^{4}+\alpha_{1}^{1} x_{1}^{3} x_{3}+ \\
\quad+\alpha_{1}^{2} x_{1}^{2} x_{2} x_{3}+\alpha_{1}^{3} x_{1} x_{2}^{2} x_{3}+\alpha_{1}^{4} x_{2}^{3} x_{3}+\alpha_{0}^{1} x_{1}^{2} x_{3}^{2}+\alpha_{0}^{2} x_{1} x_{2} x_{3}^{2}+\alpha_{0}^{3} x_{2}^{2} x_{3}^{2} .
\end{aligned}
$$

The curve $C$ given by $x_{1}=x_{2}=0$ is the only base curve of this linear system. As in Example 3.10, on the inverse image of the fiber $Y$ in the neighbourhood of the point $O=C \cap Y$ after a coordinate change $\omega=x, x_{1}=y, x_{2}=z$ and $x_{3}=1$ we have a singularity at $O$ given by the zeroes of the polynomial

$$
x^{2}+P_{2}(y, z)+P_{3}(y, z)+P_{4}(y, z)
$$

where $P_{i}$ is a homogeneous polynomial of degree $i$. If we $\operatorname{put} \operatorname{wt}(x)=1, \operatorname{wt}(y)=1$ and $\operatorname{wt}(z)=1$, it's easy to check that the singularity given by the term of the lowest weight is isolated and $\mathrm{Du}$ Val. The weights coincide with the weights of $\mathbb{A}_{1}$. Then by Theorem 2.10 in the neighbourhood of $O$ the singularity is isomorphic to $\mathbb{A}_{1} \times \mathbb{C}$, and by Corollary 2.7 the singularities of $V$ are canonical.

$\left(H_{9}\right)$ : A double cover $V \longrightarrow \mathbb{F}(2,2,2)$. The ramification divisor $D$ lies in the base point free linear system $|4 M-8 L|$ and is smooth. Hence the singularities of $V$ are canonical.

$\left(H_{10}\right)$ : A double cover $V \longrightarrow \mathbb{F}(3,0,0)$. The ramification divisor $D$ lies in the linear system $|4 M-2 L|$ and is given by the form

$$
\begin{aligned}
\alpha_{10} x_{1}^{4}+\alpha_{7}^{1} x_{1}^{3} x_{2}+\alpha_{7}^{2} x_{1}^{3} x_{3} & +\alpha_{4}^{1} x_{1}^{2} x_{2}^{2}+\alpha_{4}^{2} x_{1}^{2} x_{2} x_{3}+ \\
+ & \alpha_{4}^{3} x_{1}^{2} x_{3}^{2}+\alpha_{1}^{1} x_{1} x_{2}^{3}+\alpha_{1}^{2} x_{1} x_{2}^{2} x_{3}+\alpha_{1}^{3} x_{1} x_{2} x_{3}^{2}+\alpha_{1}^{4} x_{1} x_{3}^{3} .
\end{aligned}
$$

It is sufficient to check canonicity of the singularities of $V$ only over points of the curve $C$ given by $x_{1}=x_{2}=0$. As in Example 3.10, on the inverse image of the fiber $Y$ in the neighbourhood of the point $O=C \cap Y$ after a coordinate change $\omega=x, x_{1}=y, x_{2}=z$ and $x_{3}=1$ we have a (possible) singularity at $O$ given by the zeroes of the polynomial

$$
x^{2}+y^{3} z+y^{3}+y^{2} z^{2}+y^{2} z+y^{2}+y z^{3}+y z^{2}+y z+y
$$

and so $O$ is in fact a smooth point. Hence by Corollary 2.7 the singularities of $V$ are canonical.

$\left(H_{11}\right)$ : A double cover $V \longrightarrow \mathbb{F}(3,1,0)$. The ramification divisor $D$ lies in the linear system $|4 M-4 L|$ and is given by the form

$$
\begin{aligned}
\alpha_{8} x_{1}^{4}+\alpha_{6} x_{1}^{3} x_{2}+\alpha_{5} x_{1}^{3} x_{3} & +\alpha_{4} x_{1}^{2} x_{2}^{2}+\alpha_{3} x_{1}^{2} x_{2} x_{3}+ \\
& +\alpha_{2}^{1} x_{1}^{2} x_{3}^{2}+\alpha_{0}^{1} x_{1} x_{2} x_{3}^{2}+\alpha_{2}^{2} x_{1} x_{2}^{3}+\alpha_{1} x_{1} x_{2}^{2} x_{3}+\alpha_{0}^{2} x_{2}^{4} .
\end{aligned}
$$

The curve $C$ given by $x_{1}=x_{2}=0$ is the only base curve of this linear system. As in Example 3.10, on the inverse image of the fiber $Y$ in the neighbourhood of the 
point $O=C \cap Y$ after a coordinate change $\omega=x, x_{1}=y, x_{2}=z$ and $x_{3}=1$ we have a singularity at $O$ given by the zeroes of the polynomial

$$
x^{2}+y^{4}+y^{3} z+y^{3}+y^{2} z^{2}+y^{2} z+y^{2}+y z^{3}+y z^{2}+y z+z^{4} .
$$

If we put $\operatorname{wt}(x)=\operatorname{wt}(y)=\operatorname{wt}(z)=1$, it's easy to check that the singularity given by the term of the lowest weight is isolated and $\mathrm{Du}$ Val. The weights coincide with the weights of $\mathbb{A}_{1}$. Then by Theorem 2.10 in the neighbourhood of $O$ the singularity is isomorphic to $\mathbb{A}_{1} \times \mathbb{C}$, and by Corollary 2.7 the singularities of $V$ are canonical.

$\left(H_{12}\right)$ : A double cover $V \longrightarrow \mathbb{F}(3,1,1)$. The ramification divisor $D$ lies in the linear system $|4 M-6 L|$ and is given by the form

$$
\begin{aligned}
\alpha_{6} x_{1}^{4}+\alpha_{4}^{1} x_{1}^{3} x_{2}+\alpha_{4}^{2} x_{1}^{3} x_{3} & +\alpha_{2}^{1} x_{1}^{2} x_{2}^{2}+\alpha_{2}^{2} x_{1}^{2} x_{2} x_{3}+ \\
& +\alpha_{2}^{3} x_{1}^{2} x_{3}^{2}+\alpha_{0}^{1} x_{1} x_{2}^{3}+\alpha_{0}^{2} x_{1} x_{2}^{2} x_{3}+\alpha_{0}^{3} x_{1} x_{2} x_{3}^{2}+\alpha_{0}^{4} x_{1} x_{3}^{3} .
\end{aligned}
$$

It is sufficient to check canonicity of the singularities of $V$ only over points of the curve $C$ given by $x_{1}=x_{2}=0$. As in Example [3.10, on the inverse image of the fiber $Y$ in the neighbourhood of the point $O=C \cap Y$ after a coordinate change $\omega=x, x_{1}=y, x_{2}=z$ and $x_{3}=1$ we have a (possible) singularity at $O$ given by the zeroes of the polynomial

$$
x^{2}+y^{3} z+y^{3}+y^{2} z^{2}+y^{2} z+y^{2}+y z^{3}+y z^{2}+y z+y,
$$

and so $O$ is in fact a smooth point. Hence by Corollary [2.7 the singularities of $V$ are canonical.

$\left(H_{13}\right)$ : A double cover $V \longrightarrow \mathbb{F}(3,2,0)$. The ramification divisor $D$ lies in the linear system $|4 M-6 L|$ and is given by the form

$$
\begin{aligned}
\alpha_{6} x_{1}^{4}+\alpha_{5} x_{1}^{3} x_{2}+\alpha_{3}^{1} x_{1}^{3} x_{3}+ & \alpha_{4} x_{1}^{2} x_{2}^{2}+\alpha_{2}^{1} x_{1}^{2} x_{2} x_{3}+ \\
& +\alpha_{0}^{1} x_{1}^{2} x_{3}^{2}+\alpha_{3}^{2} x_{1} x_{2}^{3}+\alpha_{1} x_{1} x_{2}^{2} x_{3}+\alpha_{2}^{2} x_{2}^{4}+\alpha_{0}^{2} x_{2}^{3} x_{3} .
\end{aligned}
$$

The curve $C$ given by $x_{1}=x_{2}=0$ is the only base curve of this linear system. As in Example 3.10, on the inverse image of the fiber $Y$ in the neighbourhood of the point $O=C \cap Y$ after a coordinate change $\omega=x, x_{1}=y, x_{2}=z$ and $x_{3}=1$ we have a singularity at $O$ given by the zeroes of the polynomial

$$
x^{2}+y^{4}+y^{3} z+y^{3}+y^{2} z^{2}+y^{2} z+y^{2}+y z^{3}+y z^{2}+z^{4}+z^{3} .
$$

If we put $\operatorname{wt}(x)=3, \operatorname{wt}(y)=3$ and $\mathrm{wt}(z)=2$, it's easy to check that the singularity given by the term of the lowest weight is isolated and $\mathrm{Du}$ Val. The weights coincide with the weights of $\mathbb{A}_{2}$. Then by Theorem 2.10 in the neighbourhood of $O$ the singularity is isomorphic to $\mathbb{A}_{2} \times \mathbb{C}$, and by Corollary 2.7] the singularities of $V$ are canonical.

$\left(H_{14}\right)$ : A double cover $V \longrightarrow \mathbb{F}(3,2,1)$. The ramification divisor $D$ lies in the linear system $|4 M-8 L|$ and is given by the form

$$
\alpha_{4} x_{1}^{4}+\alpha_{3} x_{1}^{3} x_{2}+\alpha_{2}^{1} x_{1}^{3} x_{3}+\alpha_{2}^{2} x_{1}^{2} x_{2}^{2}+\alpha_{1}^{1} x_{1}^{2} x_{2} x_{3}+\alpha_{0}^{1} x_{1}^{2} x_{3}^{2}+\alpha_{1}^{2} x_{1} x_{2}^{3}+\alpha_{0}^{2} x_{1} x_{2}^{2} x_{3}+\alpha_{0}^{3} x_{2}^{4} .
$$

The curve $C$ given by $x_{1}=x_{2}=0$ is the only base curve of this linear system. As in Example 3.10, on the inverse image of the fiber $Y$ in the neighbourhood of the 
point $O=C \cap Y$ after a coordinate change $\omega=x, x_{1}=y, x_{2}=z$ and $x_{3}=1$ we have a singularity at $O$ given by the zeroes of the polynomial

$$
x^{2}+y^{4}+y^{3} z+y^{3}+y^{2} z^{2}+y^{2} z+y^{2}+y z^{3}+y z^{2}+z^{4} .
$$

If we put $\operatorname{wt}(x)=2, \operatorname{wt}(y)=2$ and $\operatorname{wt}(z)=1$, it's easy to check that the singularity given by the term of the lowest weight is isolated and $\mathrm{Du}$ Val. The weights coincide with the weights of $\mathbb{A}_{3}$. Then by Theorem 2.10 in the neighbourhood of $O$ the singularity is isomorphic to $\mathbb{A}_{3} \times \mathbb{C}$, and by Corollary 2.7 the singularities of $V$ are canonical.

$\left(H_{15}\right):$ A double cover $V \longrightarrow \mathbb{F}(3,3,0)$. The ramification divisor $D$ lies in the linear system $|4 M-8 L|$ and is given by the form

$$
\alpha_{4}^{1} x_{1}^{4}+\alpha_{4}^{2} x_{1}^{3} x_{2}+\alpha_{4}^{3} x_{1}^{2} x_{2}^{2}+\alpha_{4}^{4} x_{1} x_{2}^{3}+\alpha_{4}^{5} x_{2}^{4}+\alpha_{1}^{1} x_{1}^{3} x_{3}+\alpha_{1}^{2} x_{1}^{2} x_{2} x_{3}+\alpha_{1}^{3} x_{1} x_{2}^{2} x_{3}+\alpha_{1}^{4} x_{2}^{3} x_{3} .
$$

The curve $C$ given by $x_{1}=x_{2}=0$ is the only base curve of this linear system. As in Example 3.10, on the inverse image of the fiber $Y$ in the neighbourhood of the point $O=C \cap Y$ after a coordinate change $\omega=x, x_{1}=y, x_{2}=z$ and $x_{3}=1$ we have a singularity at $O$ given by the zeroes of the polynomial

$$
x^{2}+P_{3}(y, z)+P_{4}(y, z),
$$

where $P_{i}$ is a homogeneous polynomial of total degree $i$. If we put $\operatorname{wt}(x)=3$, $\mathrm{wt}(y)=2$ and $\operatorname{wt}(z)=2$, it's easy to check that the singularity given by the term of the lowest weight is isolated and $\mathrm{Du}$ Val. The weights coincide with the weights of $\mathbb{D}_{4}$. Then by Theorem 2.10 in the neighbourhood of $O$ the singularity is isomorphic to $\mathbb{D}_{4} \times \mathbb{C}$, and by Corollary 2.7 the singularities of $V$ are canonical. $\left(H_{16}\right)$ : A double cover $V \longrightarrow \mathbb{F}(3,3,1)$. The ramification divisor $D$ lies in the linear system $|4 M-10 L|$ and is given by the form

$$
\alpha_{2}^{1} x_{1}^{4}+\alpha_{2}^{2} x_{1}^{3} x_{2}+\alpha_{2}^{3} x_{1}^{2} x_{2}^{2}+\alpha_{2}^{4} x_{1} x_{2}^{3}+\alpha_{2}^{5} x_{2}^{4}+\alpha_{0}^{1} x_{1}^{3} x_{3}+\alpha_{0}^{2} x_{1}^{2} x_{2} x_{3}+\alpha_{0}^{3} x_{1} x_{2}^{2} x_{3}+\alpha_{0}^{4} x_{2}^{3} x_{3} .
$$

The curve $C$ given by $x_{1}=x_{2}=0$ is the only base curve of this linear system. As in Example 3.10, on the inverse image of the fiber $Y$ in the neighbourhood of the point $O=C \cap Y$ after a coordinate change $\omega=x, x_{1}=y, x_{2}=z$ and $x_{3}=1$ we have a singularity at $O$ given by the zeroes of the polynomial

$$
x^{2}+P_{3}(y, z)+P_{4}(y, z),
$$

where $P_{i}$ is a homogeneous polynomial of total degree $i$. If we put $\operatorname{wt}(x)=3$, $\mathrm{wt}(y)=2$ and $\operatorname{wt}(z)=2$, it's easy to check that the singularity given by the term of the lowest weight is isolated and Du Val. The weights coincide with the weights of $\mathbb{D}_{4}$. Then by Theorem 2.10 in the neighbourhood of $O$ the singularity is isomorphic to $\mathbb{D}_{4} \times \mathbb{C}$, and by Corollary 2.7 the singularities of $V$ are canonical. $\left(H_{17}\right)$ : A double cover $V \longrightarrow \mathbb{F}(4,0,0)$. The ramification divisor $D$ lies in the linear system $|4 M-4 L|$ and is given by the form

$$
\begin{aligned}
\alpha_{12} x_{1}^{4}+\alpha_{8}^{1} x_{1}^{3} x_{2}+\alpha_{8}^{2} x_{1}^{3} x_{3} & +\alpha_{4}^{1} x_{1}^{2} x_{2}^{2}+\alpha_{4}^{2} x_{1}^{2} x_{2} x_{3}+ \\
& +\alpha_{4}^{3} x_{1}^{2} x_{3}^{2}+\alpha_{0}^{1} x_{1} x_{2}^{3}+\alpha_{0}^{2} x_{1} x_{2}^{2} x_{3}+\alpha_{0}^{3} x_{1} x_{2} x_{3}^{2}+\alpha_{0}^{4} x_{1} x_{3}^{3} .
\end{aligned}
$$

It is sufficient to check canonicity of the singularities of $V$ only over points of the curve $C$ given by $x_{1}=x_{2}=0$. As in Example 3.10, on the inverse image of the fiber $Y$ in the neighbourhood of the point $O=C \cap Y$ after a coordinate change 
$\omega=x, x_{1}=y, x_{2}=z$ and $x_{3}=1$ we have a (possible) singularity at $O$ given by the zeroes of the polynomial

$$
x^{2}+y^{3} z+y^{3}+y^{2} z^{2}+y^{2} z+y^{2}+y z^{3}+y z^{2}+y z+y
$$

and so $O$ is in fact a smooth point. Hence by Corollary 2.7 the singularities of $V$ are canonical.

$\left(H_{18}\right)$ : A double cover $V \longrightarrow \mathbb{F}(4,1,0)$. The ramification divisor $D$ lies in the linear system $|4 M-6 L|$ and is given by the form

$$
\alpha_{10} x_{1}^{4}+\alpha_{8} x_{1}^{3} x_{2}+\alpha_{6} x_{1}^{3} x_{3}+\alpha_{4} x_{1}^{2} x_{2}^{2}+\alpha_{3} x_{1}^{2} x_{2} x_{3}+\alpha_{2} x_{1}^{2} x_{3}^{2}+\alpha_{1} x_{1} x_{2}^{3}+\alpha_{0} x_{1} x_{2}^{2} x_{3} .
$$

It is sufficient to check canonicity of the singularities of $V$ only over points of the curve $C$ given by $x_{1}=x_{2}=0$. As in Example 3.10, on the inverse image of the fiber $Y$ in the neighbourhood of the point $O=C \cap Y$ after a coordinate change $\omega=x, x_{1}=y, x_{2}=z$ and $x_{3}=1$ we have a singularity at $O$ given by the zeroes of the polynomial

$$
x^{2}+y^{4}+y^{3} z+y^{3}+y^{2} z^{2}+y^{2} z+y^{2}+y z^{3}+y z^{2} .
$$

If we put $\operatorname{wt}(x)=2, \operatorname{wt}(y)=2$ and $\operatorname{wt}(z)=1$, it's easy to check that the singularity given by the term of the lowest weight is isolated and $\mathrm{Du}$ Val. The weights coincide with the weights of $\mathbb{A}_{3}$. Then by Theorem 2.10 in the neighbourhood of $O$ the singularity is isomorphic to $\mathbb{A}_{3} \times \mathbb{C}$, and by Corollary 2.7 the singularities of $V$ are canonical.

$\left(H_{19}\right)$ : A double cover $V \longrightarrow \mathbb{F}(4,2,0)$. The ramification divisor $D$ lies in the linear system $|4 M-8 L|$ and is given by the form

$$
\alpha_{8} x_{1}^{4}+\alpha_{6} x_{1}^{3} x_{2}+\alpha_{4} x_{1}^{3} x_{3}+\alpha_{4} x_{1}^{2} x_{2}^{2}+\alpha_{2} x_{1}^{2} x_{2} x_{3}+\alpha_{0} x_{1}^{2} x_{3}^{2}+\alpha_{2} x_{1} x_{2}^{3}+\alpha_{0} x_{1} x_{2}^{2} x_{3}+\alpha_{0} x_{2}^{4} .
$$

The curve $C$ given by $x_{1}=x_{2}=0$ is the only base curve of this linear system. As in Example 3.10, on the inverse image of the fiber $Y$ in the neighbourhood of the point $O=C \cap Y$ after a coordinate change $\omega=x, x_{1}=y, x_{2}=z$ and $x_{3}=1$ we have a singularity at $O$ given by the zeroes of the polynomial

$$
x^{2}+y^{4}+y^{3} z+y^{3}+y^{2} z^{2}+y^{2} z+y^{2}+y z^{3}+y z^{2}+z^{4} .
$$

If we put $\operatorname{wt}(x)=2, \operatorname{wt}(y)=2$ and $\operatorname{wt}(z)=1$, it's easy to check that the singularity given by the term of the lowest weight is isolated and $\mathrm{Du}$ Val. The weights coincide with the weights of $\mathbb{A}_{3}$. Then by Theorem 2.10 in the neighbourhood of $O$ the singularity is isomorphic to $\mathbb{A}_{3} \times \mathbb{C}$, and by Corollary 2.7 the singularities of $V$ are canonical.

$\left(H_{20}\right)$ : A double cover $V \longrightarrow \mathbb{F}(4,2,1)$. The ramification divisor $D$ lies in the linear system $|4 M-10 L|$ and is given by the form

$$
\alpha_{6} x_{1}^{4}+\alpha_{4} x_{1}^{3} x_{2}+\alpha_{3} x_{1}^{3} x_{3}+\alpha_{2} x_{1}^{2} x_{2}^{2}+\alpha_{1} x_{1}^{2} x_{2} x_{3}+\alpha_{0}^{1} x_{1} x_{2}^{3}+\alpha_{0}^{2} x_{1}^{2} x_{3}^{2} .
$$

It is sufficient to check canonicity of the singularities of $V$ only over points of the curve $C$ given by $x_{1}=x_{2}=0$. As in Example 3.10, on the inverse image of the fiber $Y$ in the neighbourhood of the point $O=C \cap Y$ after a coordinate change $\omega=x, x_{1}=y, x_{2}=z$ and $x_{3}=1$ we have a singularity at $O$ given by the zeroes of the polynomial

$$
x^{2}+y^{4}+y^{3} z+y^{3}+y^{2} z^{2}+y^{2} z+y z^{3}+y^{2} .
$$


If we put $\operatorname{wt}(x)=3, \operatorname{wt}(y)=3$ and $\operatorname{wt}(z)=1$, it's easy to check that the singularity given by the term of the lowest weight is isolated and Du Val. The weights coincide with the weights of $\mathbb{A}_{5}$. Then by Theorem 2.10 in the neighbourhood of $O$ the singularity is isomorphic to $\mathbb{A}_{5} \times \mathbb{C}$, and by Corollary 2.7 the singularities of $V$ are canonical.

$\left(H_{21}\right)$ : A double cover $V \longrightarrow \mathbb{F}(4,3,0)$. The ramification divisor $D$ lies in the linear system $|4 M-10 L|$ and is given by the form

$$
\alpha_{6} x_{1}^{4}+\alpha_{5} x_{1}^{3} x_{2}+\alpha_{4} x_{1}^{2} x_{2}^{2}+\alpha_{3} x_{1} x_{2}^{3}+\alpha_{2}^{1} x_{2}^{4}+\alpha_{0}^{2} x_{1}^{3} x_{3}+\alpha_{1} x_{1}^{2} x_{2} x_{3}+\alpha_{0} x_{1} x_{2}^{2} x_{3} .
$$

The curve $C$ given by $x_{1}=x_{2}=0$ is the only base curve of this linear system. As in Example 3.10, on the inverse image of the fiber $Y$ in the neighbourhood of the point $O=C \cap Y$ after a coordinate change $\omega=x, x_{1}=y, x_{2}=z$ and $x_{3}=1$ we have a singularity at $O$ given by the zeroes of the polynomial

$$
x^{2}+y^{4}+y^{3} z+y^{2} z^{2}+y z^{3}+z^{4}+y^{3}+y^{2} z+y z^{2} .
$$

If we put $\operatorname{wt}(x)=3, \operatorname{wt}(y)=2$ and $\operatorname{wt}(z)=2$, it's easy to check that the singularity given by the term of the lowest weight is isolated and $\mathrm{Du}$ Val. The weights coincide with the weights of $\mathbb{D}_{4}$. Then by Theorem 2.10 in the neighbourhood of $O$ the singularity is isomorphic to $\mathbb{D}_{4} \times \mathbb{C}$, and by Corollary 2.7 the singularities of $V$ are canonical.

$\left(H_{22}\right)$ : A double cover $V \longrightarrow \mathbb{F}(4,3,1)$. The ramification divisor $D$ lies in the linear system $|4 M-12 L|$ and is given by the form

$$
\alpha_{4} x_{1}^{4}+\alpha_{3}^{1} x_{1}^{3} x_{2}+\alpha_{1}^{1} x_{1}^{3} x_{3}+\alpha_{2} x_{1}^{2} x_{2}^{2}+\alpha_{0}^{1} x_{1}^{2} x_{2} x_{3}+\alpha_{1}^{2} x_{1} x_{2}^{3}+\alpha_{0}^{2} x_{2}^{4}
$$

The curve $C$ given by $x_{1}=x_{2}=0$ is the only base curve of this linear system. As in Example 3.10, on the inverse image of the fiber $Y$ in the neighbourhood of the point $O=C \cap Y$ after a coordinate change $\omega=x, x_{1}=y, x_{2}=z$ and $x_{3}=1$ we have a singularity at $O$ given by the zeroes of the polynomial

$$
x^{2}+y^{4}+y^{3} z+y^{3}+y^{2} z^{2}+y^{2} z+y z^{3}+z^{4} .
$$

If we put $\operatorname{wt}(x)=4, \operatorname{wt}(y)=3$ and wt $(z)=2$, it's easy to check that the singularity given by the term of the lowest weight is isolated and Du Val. The weights coincide with the weights of $\mathbb{D}_{5}$. Then by Theorem 2.10 in the neighbourhood of $O$ the singularity is isomorphic to $\mathbb{D}_{5} \times \mathbb{C}$, and by Corollary 2.7 the singularities of $V$ are canonical.

$\left(H_{23}\right)$ : A double cover $V \longrightarrow \mathbb{F}(4,4,0)$. The ramification divisor $D$ lies in the linear system $|4 M-12 L|$ and is given by the form

$$
\alpha_{4}^{1} x_{1}^{4}+\alpha_{4}^{2} x_{1}^{3} x_{2}+\alpha_{4}^{3} x_{1}^{2} x_{2}^{2}+\alpha_{4}^{4} x_{1} x_{2}^{3}+\alpha_{4}^{5} x_{2}^{4}+\alpha_{0}^{1} x_{1}^{3} x_{3}+\alpha_{0}^{2} x_{1}^{2} x_{2} x_{3}+\alpha_{0}^{3} x_{1} x_{2}^{2} x_{3}+\alpha_{0}^{4} x_{2}^{3} x_{3} .
$$

The curve $C$ given by $x_{1}=x_{2}=0$ is the only base curve of this linear system. As in Example 3.10, on the inverse image of the fiber $Y$ in the neighbourhood of the point $O=C \cap Y$ after a coordinate change $\omega=x, x_{1}=y, x_{2}=z$ and $x_{3}=1$ we have a singularity at $O$ given by the zeroes of the polynomial

$$
x^{2}+P_{3}(y, z)+P_{4}(y, z)
$$

where $P_{i}$ is a homogeneous polynomial of total degree $i$. If we put $\operatorname{wt}(x)=3$, $\operatorname{wt}(y)=2$ and $\operatorname{wt}(z)=2$, it's easy to check that the singularity given by the term of the lowest weight is isolated and Du Val. The weights coincide with the 
weights of $\mathbb{D}_{4}$. Then by Theorem 2.10 in the neighbourhood of $O$ the singularity is isomorphic to $\mathbb{D}_{4} \times \mathbb{C}$, and by Corollary 2.7 the singularities of $V$ are canonical. $\left(H_{24}\right)$ : A double cover $V \longrightarrow \mathbb{F}(5,1,0)$. The ramification divisor $D$ lies in the linear system $|4 M-8 L|$ and is given by the form

$$
\alpha_{12} x_{1}^{4}+\alpha_{8} x_{1}^{3} x_{2}+\alpha_{7} x_{1}^{3} x_{3}+\alpha_{4} x_{1}^{2} x_{2}^{2}+\alpha_{3} x_{1}^{2} x_{2} x_{3}+\alpha_{0} x_{1} x_{2}^{3}+\alpha_{2} x_{1}^{2} x_{3}^{2} .
$$

It is sufficient to check canonicity of the singularities of $V$ only over points of the curve $C$ given by $x_{1}=x_{2}=0$. As in Example 3.10, on the inverse image of the fiber $Y$ in the neighbourhood of the point $O=C \cap Y$ after a coordinate change $\omega=x, x_{1}=y, x_{2}=z$ and $x_{3}=1$ we have a singularity at $O$ given by the zeroes of the polynomial

$$
x^{2}+y^{4}+y^{3} z+y^{3}+y^{2} z^{2}+y^{2} z+y z^{3}+y^{2} .
$$

If we put $\operatorname{wt}(x)=3, \operatorname{wt}(y)=3$ and wt $(z)=1$, it's easy to check that the singularity given by the term of the lowest weight is isolated and $\mathrm{Du}$ Val. The weights coincide with the weights of $\mathbb{A}_{5}$. Then by Theorem 2.10 in the neighbourhood of $O$ the singularity is isomorphic to $\mathbb{A}_{5} \times \mathbb{C}$, and by Corollary 2.7 the singularities of $V$ are canonical.

$\left(H_{25}\right)$ : A double cover $V \longrightarrow \mathbb{F}(5,2,0)$. The ramification divisor $D$ lies in the linear system $|4 M-10 L|$ and is given by the form

$$
\alpha_{10} x_{1}^{4}+\alpha_{7} x_{1}^{3} x_{2}+\alpha_{5} x_{1}^{3} x_{3}+\alpha_{4} x_{1}^{2} x_{2}^{2}+\alpha_{0}^{1} x_{1}^{2} x_{2} x_{3}+\alpha_{1} x_{1} x_{2}^{3}+\alpha_{0}^{2} x_{1}^{2} x_{3}^{2} .
$$

The curve $C$ given by $x_{1}=x_{2}=0$ is the only base curve of this linear system. As in Example 3.10, on the inverse image of the fiber $Y$ in the neighbourhood of the point $O=C \cap Y$ after a coordinate change $\omega=x, x_{1}=y, x_{2}=z$ and $x_{3}=1$ we have a singularity at $O$ given by the zeroes of the polynomial

$$
x^{2}+y^{4}+y^{3} z+y^{3}+y^{2} z^{2}+y^{2} z+y z^{3}+y^{2} .
$$

If we put $\operatorname{wt}(x)=3, \operatorname{wt}(y)=3$ and $\operatorname{wt}(z)=1$, it's easy to check that the singularity given by the term of the lowest weight is isolated and $\mathrm{Du}$ Val. The weights coincide with the weights of $\mathbb{A}_{5}$. Then by Theorem 2.10 in the neighbourhood of $O$ the singularity is isomorphic to $\mathbb{A}_{5} \times \mathbb{C}$, and by Corollary 2.7 the singularities of $V$ are canonical.

$\left(H_{26}\right)$ : A double cover $V \longrightarrow \mathbb{F}(5,3,0)$. The ramification divisor $D$ lies in the linear system $|4 M-12 L|$ and is given by the form

$$
\alpha_{8} x_{1}^{4}+\alpha_{6} x_{1}^{3} x_{2}+\alpha_{3} x_{1}^{3} x_{3}+\alpha_{4} x_{1}^{2} x_{2}^{2}+\alpha_{1}^{1} x_{1}^{2} x_{2} x_{3}+\alpha_{1}^{2} x_{1} x_{2}^{3}+\alpha_{0} x_{2}^{4}
$$

The curve $C$ given by $x_{1}=x_{2}=0$ is the only base curve of this linear system. As in Example 3.10, on the inverse image of the fiber $Y$ in the neighbourhood of the point $O=C \cap Y$ after a coordinate change $\omega=x, x_{1}=y, x_{2}=z$ and $x_{3}=1$ we have a singularity at $O$ given by the zeroes of the polynomial

$$
x^{2}+y^{4}+y^{3} z+y^{3}+y^{2} z^{2}+y^{2} z+y z^{3}+z^{4} .
$$

If we put $\operatorname{wt}(x)=4, \operatorname{wt}(y)=3$ and $\operatorname{wt}(z)=2$, it's easy to check that the singularity given by the term of the lowest weight is isolated and Du Val. The weights coincide with the weights of $\mathbb{D}_{5}$. Then by Theorem 2.10 in the neighbourhood of $O$ the singularity is isomorphic to $\mathbb{D}_{5} \times \mathbb{C}$, and by Corollary 2.7 the singularities of $V$ are canonical. 
$\left(H_{27}\right)$ : A double cover $V \longrightarrow \mathbb{F}(5,3,1)$. The ramification divisor $D$ lies in the linear system $|4 M-14 L|$ and is given by the form

$$
\alpha_{6} x_{1}^{4}+\alpha_{4} x_{1}^{3} x_{2}+\alpha_{2}^{1} x_{1}^{3} x_{3}+\alpha_{2}^{2} x_{1}^{2} x_{2}^{2}+\alpha_{0}^{1} x_{1}^{2} x_{2} x_{3}+\alpha_{0}^{2} x_{1} x_{2}^{3} .
$$

It is sufficient to check canonicity of the singularities of $V$ only over points of the curve $C$ given by $x_{1}=x_{2}=0$. As in Example 3.10, on the inverse image of the fiber $Y$ in the neighbourhood of the point $O=C \cap Y$ after a coordinate change $\omega=x, x_{1}=y, x_{2}=z$ and $x_{3}=1$ we have a singularity at $O$ given by the zeroes of the polynomial

$$
x^{2}+y^{4}+y^{3} z+y^{3}+y^{2} z^{2}+y^{2} z+y z^{3} .
$$

If we put $\operatorname{wt}(x)=5, \operatorname{wt}(y)=4$ and $\operatorname{wt}(z)=2$, it's easy to check that the singularity given by the term of the lowest weight is isolated and $\mathrm{Du}$ Val. The weights coincide with the weights of $\mathbb{D}_{6}$. Then by Theorem 2.10 in the neighbourhood of $O$ the singularity is isomorphic to $\mathbb{D}_{6} \times \mathbb{C}$, and by Corollary 2.7 the singularities of $V$ are canonical.

$\left(H_{28}\right)$ : A double cover $V \longrightarrow \mathbb{F}(5,4,0)$. The ramification divisor $D$ lies in the linear system $|4 M-14 L|$ and is given by the form

$$
\alpha_{6} x_{1}^{4}+\alpha_{5} x_{1}^{3} x_{2}+\alpha_{1} x_{1}^{3} x_{3}+\alpha_{4} x_{1}^{2} x_{2}^{2}+\alpha_{0} x_{1}^{2} x_{2} x_{3}+\alpha_{3} x_{1} x_{2}^{3}+\alpha_{2} x_{2}^{4}
$$

The curve $C$ given by $x_{1}=x_{2}=0$ is the only base curve of this linear system. As in Example 3.10, on the inverse image of the fiber $Y$ in the neighbourhood of the point $O=C \cap Y$ after a coordinate change $\omega=x, x_{1}=y, x_{2}=z$ and $x_{3}=1$ we have a singularity at $O$ given by the zeroes of the polynomial

$$
x^{2}+y^{4}+y^{3} z+y^{3}+y^{2} z^{2}+y^{2} z+y z^{3}+z^{4} .
$$

If we put $\mathrm{wt}(x)=4, \operatorname{wt}(y)=3$ and $\operatorname{wt}(z)=2$, it's easy to check that the singularity given by the term of the lowest weight is isolated and $\mathrm{Du}$ Val. The weights coincide with the weights of $\mathbb{D}_{5}$. Then by Theorem 2.10 in the neighbourhood of $O$ the singularity is isomorphic to $\mathbb{D}_{5} \times \mathbb{C}$, and by Corollary 2.7 the singularities of $V$ are canonical.

$\left(H_{29}\right)$ : A double cover $V \longrightarrow \mathbb{F}(5,4,1)$. The ramification divisor $D$ lies in the linear system $|4 M-16 L|$ and is given by the form

$$
\alpha_{4} x_{1}^{4}+\alpha_{3} x_{1}^{3} x_{2}+\alpha_{0}^{1} x_{1}^{3} x_{3}+\alpha_{2} x_{1}^{2} x_{2}^{2}+\alpha_{1} x_{1} x_{2}^{3}+\alpha_{0}^{2} x_{2}^{4} .
$$

The curve $C$ given by $x_{1}=x_{2}=0$ is the only base curve of this linear system. As in Example 3.10, on the inverse image of the fiber $Y$ in the neighbourhood of the point $O=C \cap Y$ after a coordinate change $\omega=x, x_{1}=y, x_{2}=z$ and $x_{3}=1$ we have a singularity at $O$ given by the zeroes of the polynomial

$$
x^{2}+y^{4}+y^{3} z+y^{3}+y^{2} z^{2}+y z^{3}+z^{4} .
$$

If we put $\operatorname{wt}(x)=6, \operatorname{wt}(y)=4$ and $\mathrm{wt}(z)=3$, it's easy to check that the singularity given by the term of the lowest weight is isolated and $\mathrm{Du}$ Val. The weights coincide with the weights of $\mathbb{E}_{6}$. Then by Theorem 2.10 in the neighbourhood of $O$ the singularity is isomorphic to $\mathbb{E}_{6} \times \mathbb{C}$, and by Corollary 2.7 the singularities of $V$ are canonical.

$\left(H_{30}\right)$ : A double cover $V \longrightarrow \mathbb{F}(6,2,0)$. The ramification divisor $D$ lies in the linear system $|4 M-12 L|$ and is given by the form

$$
\alpha_{12} x_{1}^{4}+\alpha_{8} x_{1}^{3} x_{2}+\alpha_{6} x_{1}^{3} x_{3}+\alpha_{4} x_{1}^{2} x_{2}^{2}+\alpha_{2} x_{1}^{2} x_{2} x_{3}+\alpha_{0}^{1} x_{1}^{2} x_{3}^{2}+\alpha_{0}^{2} x_{1} x_{2}^{3} .
$$


It is sufficient to check canonicity of the singularities of $V$ only over points of the curve $C$ given by $x_{1}=x_{2}=0$. As in Example 3.10, on the inverse image of the fiber $Y$ in the neighbourhood of the point $O=C \cap Y$ after a coordinate change $\omega=x, x_{1}=y, x_{2}=z$ and $x_{3}=1$ we have a singularity at $O$ given by the zeroes of the polynomial

$$
x^{2}+y^{4}+y^{3} z+y^{3}+y^{2} z^{2}+y^{2} z+y^{2}+y z^{3} .
$$

If we put $\operatorname{wt}(x)=3, \operatorname{wt}(y)=3$ and $\operatorname{wt}(z)=1$, it's easy to check that the singularity given by the term of the lowest weight is isolated and Du Val. The weights coincide with the weights of $\mathbb{A}_{5}$. Then by Theorem 2.10 in the neighbourhood of $O$ the singularity is isomorphic to $\mathbb{A}_{5} \times \mathbb{C}$, and by Corollary 2.7 the singularities of $V$ are canonical.

$\left(H_{31}\right)$ : A double cover $V \longrightarrow \mathbb{F}(6,3,0)$. The ramification divisor $D$ lies in the linear system $|4 M-14 L|$ and is given by the form

$$
\alpha_{10} x_{1}^{4}+\alpha_{7} x_{1}^{3} x_{2}+\alpha_{4} x_{1}^{3} x_{3}+\alpha_{2} x_{1}^{2} x_{2}^{2}+\alpha_{0} x_{1}^{2} x_{2} x_{3}+\alpha_{1} x_{1} x_{2}^{3} .
$$

It is sufficient to check canonicity of the singularities of $V$ only over points of the curve $C$ given by $x_{1}=x_{2}=0$. As in Example 3.10, on the inverse image of the fiber $Y$ in the neighbourhood of the point $O=C \cap Y$ after a coordinate change $\omega=x, x_{1}=y, x_{2}=z$ and $x_{3}=1$ we have a singularity at $O$ given by the zeroes of the polynomial

$$
x^{2}+y^{4}+y^{3} z+y^{3}+y^{2} z^{2}+y^{2} z+y z^{3} .
$$

If we put $\operatorname{wt}(x)=5, \operatorname{wt}(y)=4$ and $\operatorname{wt}(z)=2$, it's easy to check that the singularity given by the term of the lowest weight is isolated and $\mathrm{Du}$ Val. The weights coincide with the weights of $\mathbb{D}_{6}$. Then by Theorem 2.10 in the neighbourhood of $O$ the singularity is isomorphic to $\mathbb{D}_{6} \times \mathbb{C}$, and by Corollary 2.7 the singularities of $V$ are canonical.

$\left(H_{32}\right)$ : A double cover $V \longrightarrow \mathbb{F}(6,4,0)$. The ramification divisor $D$ lies in the linear system $|4 M-16 L|$ and is given by the form

$$
\alpha_{8} x_{1}^{4}+\alpha_{6} x_{1}^{3} x_{2}+\alpha_{2} x_{1}^{3} x_{3}+\alpha_{4} x_{1}^{2} x_{2}^{2}+\alpha_{0}^{1} x_{1}^{2} x_{2} x_{3}+\alpha_{0}^{2} x_{2}^{4} .
$$

The curve $C$ given by $x_{1}=x_{2}=0$ is the only base curve of this linear system. As in Example 3.10, on the inverse image of the fiber $Y$ in the neighbourhood of the point $O=C \cap Y$ after a coordinate change $\omega=x, x_{1}=y, x_{2}=z$ and $x_{3}=1$ we have a singularity at $O$ given by the zeroes of the polynomial

$$
x^{2}+y^{4}+y^{3} z+y^{3}+y^{2} z^{2}+y^{2} z+z^{4} .
$$

If we put $\operatorname{wt}(x)=4, \operatorname{wt}(y)=3$ and $\operatorname{wt}(z)=2$, it's easy to check that the singularity given by the term of the lowest weight is isolated and $\mathrm{Du}$ Val. The weights coincide with the weights of $\mathbb{D}_{5}$. Then by Theorem 2.10 in the neighbourhood of $O$ the singularity is isomorphic to $\mathbb{D}_{5} \times \mathbb{C}$, and by Corollary 2.7 the singularities of $V$ are canonical.

$\left(H_{33}\right)$ : A double cover $V \longrightarrow \mathbb{F}(6,4,1)$. The ramification divisor $D$ lies in the linear system $|4 M-18 L|$ and is given by the form

$$
\alpha_{6} x_{1}^{4}+\alpha_{4} x_{1}^{3} x_{2}+\alpha_{0}^{1} x_{1}^{3} x_{3}+\alpha_{2} x_{1}^{2} x_{2}^{2}+\alpha_{0}^{2} x_{1} x_{2}^{3} .
$$

It is sufficient to check canonicity of the singularities of $V$ only over points of the curve $C$ given by $x_{1}=x_{2}=0$. As in Example [3.10, on the inverse image of the 
fiber $Y$ in the neighbourhood of the point $O=C \cap Y$ after a coordinate change $\omega=x, x_{1}=y, x_{2}=z$ and $x_{3}=1$ we have a singularity at $O$ given by the zeroes of the polynomial

$$
x^{2}+y^{4}+y^{3} z+y^{3}+y^{2} z^{2}+y z^{3} .
$$

If we put $\operatorname{wt}(x)=9, \operatorname{wt}(y)=6$ and $\operatorname{wt}(z)=4$, it's easy to check that the singularity given by the term of the lowest weight is isolated and Du Val. The weights coincide with the weights of $\mathbb{E}_{7}$. Then by Theorem 2.10 in the neighbourhood of $O$ the singularity is isomorphic to $\mathbb{E}_{7} \times \mathbb{C}$, and by Corollary 2.7 the singularities of $V$ are canonical.

$\left(H_{34}\right):$ A double cover $V \longrightarrow \mathbb{F}(6,5,0)$. The ramification divisor $D$ lies in the linear system $|4 M-18 L|$ and is given by the form

$$
\alpha_{6} x_{1}^{4}+\alpha_{5} x_{1}^{3} x_{2}+\alpha_{0} x_{1}^{3} x_{3}+\alpha_{4} x_{1}^{2} x_{2}^{2}+\alpha_{3} x_{1} x_{2}^{3}+\alpha_{2} x_{2}^{4}
$$

The curve $C$ given by $x_{1}=x_{2}=0$ is the only base curve of this linear system. As in Example 3.10, on the inverse image of the fiber $Y$ in the neighbourhood of the point $O=C \cap Y$ after a coordinate change $\omega=x, x_{1}=y, x_{2}=z$ and $x_{3}=1$ we have a singularity at $O$ given by the zeroes of the polynomial

$$
x^{2}+y^{4}+y^{3} z+y^{3}+y^{2} z^{2}+y z^{3}+z^{4} .
$$

If we put $\operatorname{wt}(x)=6, \operatorname{wt}(y)=4$ and $\operatorname{wt}(z)=3$, it's easy to check that the singularity given by the term of the lowest weight is isolated and $\mathrm{Du}$ Val. The weights coincide with the weights of $\mathbb{E}_{6}$. Then by Theorem 2.10 in the neighbourhood of $O$ the singularity is isomorphic to $\mathbb{E}_{6} \times \mathbb{C}$, and by Corollary 2.7 the singularities of $V$ are canonical.

$\left(H_{35}\right)$ : A double cover $V \longrightarrow \mathbb{F}(7,3,0)$. The ramification divisor $D$ lies in the linear system $|4 M-16 L|$ and is given by the form

$$
\alpha_{12} x_{1}^{4}+\alpha_{8} x_{1}^{3} x_{2}+\alpha_{5} x_{1}^{3} x_{3}+\alpha_{4} x_{1}^{2} x_{2}^{2}+\alpha_{1} x_{1}^{2} x_{2} x_{3}+\alpha_{0} x_{1} x_{2}^{3} .
$$

It is sufficient to check canonicity of the singularities of $V$ only over points of the curve $C$ given by $x_{1}=x_{2}=0$. As in Example 3.10, on the inverse image of the fiber $Y$ in the neighbourhood of the point $O=C \cap Y$ after a coordinate change $\omega=x, x_{1}=y, x_{2}=z$ and $x_{3}=1$ we have a singularity at $O$ given by the zeroes of the polynomial

$$
x^{2}+y^{4}+y^{3} z+y^{3}+y^{2} z^{2}+y^{2} z+y z^{3} .
$$

If we put $\operatorname{wt}(x)=5, \operatorname{wt}(y)=4$ and $\mathrm{wt}(z)=2$, it's easy to check that the singularity given by the term of the lowest weight is isolated and Du Val. The weights coincide with the weights of $\mathbb{D}_{6}$. Then by Theorem 2.10 in the neighbourhood of $O$ the singularity is isomorphic to $\mathbb{D}_{6} \times \mathbb{C}$, and by Corollary 2.7] the singularities of $V$ are canonical.

$\left(H_{36}\right)$ : A double cover $V \longrightarrow \mathbb{F}(7,4,0)$. The ramification divisor $D$ lies in the linear system $|4 M-18 L|$ and is given by the form

$$
\alpha_{10} x_{1}^{4}+\alpha_{7} x_{1}^{3} x_{2}+\alpha_{3} x_{1}^{3} x_{3}+\alpha_{4} x_{1}^{2} x_{2}^{2}+\alpha_{0} x_{1}^{2} x_{2} x_{3}+\alpha_{1} x_{1} x_{2}^{3} .
$$

It is sufficient to check canonicity of the singularities of $V$ only over points of the curve $C$ given by $x_{1}=x_{2}=0$. As in Example 3.10, on the inverse image of the fiber $Y$ in the neighbourhood of the point $O=C \cap Y$ after a coordinate change 
$\omega=x, x_{1}=y, x_{2}=z$ and $x_{3}=1$ we have a singularity at $O$ given by the zeroes of the polynomial

$$
x^{2}+y^{4}+y^{3} z+y^{3}+y^{2} z^{2}+y^{2} z+y z^{3} .
$$

If we put $\operatorname{wt}(x)=5, \operatorname{wt}(y)=4$ and $\operatorname{wt}(z)=2$, it's easy to check that the singularity given by the term of the lowest weight is isolated and $\mathrm{Du}$ Val. The weights coincide with the weights of $\mathbb{D}_{6}$. Then by Theorem 2.10 in the neighbourhood of $O$ the singularity is isomorphic to $\mathbb{D}_{6} \times \mathbb{C}$, and by Corollary 2.7 the singularities of $V$ are canonical.

$\left(H_{37}\right)$ : A double cover $V \longrightarrow \mathbb{F}(7,5,0)$. The ramification divisor $D$ lies in the linear system $|4 M-20 L|$ and is given by the form

$$
\alpha_{8} x_{1}^{4}+\alpha_{6} x_{1}^{3} x_{2}+\alpha_{1} x_{1}^{3} x_{3}+\alpha_{4} x_{1}^{2} x_{2}^{2}+\alpha_{2} x_{1} x_{2}^{3}+\alpha_{0} x_{2}^{4}
$$

The curve $C$ given by $x_{1}=x_{2}=0$ is the only base curve of this linear system. As in Example 3.10, on the inverse image of the fiber $Y$ in the neighbourhood of the point $O=C \cap Y$ after a coordinate change $\omega=x, x_{1}=y, x_{2}=z$ and $x_{3}=1$ we have a singularity at $O$ given by the zeroes of the polynomial

$$
x^{2}+y^{4}+y^{3} z+y^{3}+y^{2} z^{2}+y z^{3}+z^{4} .
$$

If we put $\operatorname{wt}(x)=6, \operatorname{wt}(y)=4$ and $\operatorname{wt}(z)=3$, it is easy to check that the singularity given by the term of the lowest weight is isolated and Du Val. The weights coincide with the weights of $\mathbb{E}_{6}$. Then in the neighbourhood of $O$ the singularity is isomorphic to $\mathbb{E}_{6} \times \mathbb{C}$ by Theorem 2.10 . Hence, the singularities of $V$ are canonical by Corollary 2.7

$\left(H_{38}\right)$ : A double cover $V \longrightarrow \mathbb{F}(7,5,1)$. The ramification divisor $D$ lies in the linear system $|4 M-22 L|$ and is given by the form

$$
\alpha_{6} x_{1}^{4}+\alpha_{4} x_{1}^{3} x_{2}+\alpha_{0}^{1} x_{1}^{3} x_{3}+\alpha_{2} x_{1}^{2} x_{2}^{2}+\alpha_{0}^{2} x_{1} x_{2}^{3} .
$$

It is sufficient to check canonicity of the singularities of $V$ only over points of the curve $C$ given by $x_{1}=x_{2}=0$. As in Example 3.10, on the inverse image of the fiber $Y$ in the neighbourhood of the point $O=C \cap Y$ after a coordinate change $\omega=x, x_{1}=y, x_{2}=z$ and $x_{3}=1$ we have a singularity at $O$ given by the zeroes of the polynomial

$$
x^{2}+y^{4}+y^{3} z+y^{3}+y^{2} z^{2}+y z^{3} .
$$

If we put $\operatorname{wt}(x)=9, \operatorname{wt}(y)=6$ and $\operatorname{wt}(z)=4$, it's easy to check that the singularity given by the term of the lowest weight is isolated and Du Val. The weights coincide with the weights of $\mathbb{E}_{7}$. Then by Theorem 2.10 in the neighbourhood of $O$ the singularity is isomorphic to $\mathbb{E}_{7} \times \mathbb{C}$, and by Corollary 2.7 the singularities of $V$ are canonical.

$\left(H_{39}\right)$ : A double cover $V \longrightarrow \mathbb{F}(8,4,0)$. The ramification divisor $D$ lies in the linear system $|4 M-20 L|$ and is given by the form

$$
\alpha_{12} x_{1}^{4}+\alpha_{8} x_{1}^{3} x_{2}+\alpha_{4}^{1} x_{1}^{3} x_{3}+\alpha_{4}^{2} x_{1}^{2} x_{2}^{2}+\alpha_{0}^{1} x_{1}^{2} x_{2} x_{3}+\alpha_{0}^{2} x_{1} x_{2}^{3} .
$$

It is sufficient to check canonicity of the singularities of $V$ only over points of the curve $C$ given by $x_{1}=x_{2}=0$. As in Example 3.10, on the inverse image of the fiber $Y$ in the neighbourhood of the point $O=C \cap Y$ after a coordinate change 
$\omega=x, x_{1}=y, x_{2}=z$ and $x_{3}=1$ we have a singularity at $O$ given by the zeroes of the polynomial

$$
x^{2}+y^{4}+y^{3} z+y^{3}+y^{2} z^{2}+y^{2} z+y z^{3} .
$$

If we put $\operatorname{wt}(x)=5, \operatorname{wt}(y)=4$ and $\operatorname{wt}(z)=2$, it's easy to check that the singularity given by the term of the lowest weight is isolated and $\mathrm{Du}$ Val. The weights coincide with the weights of $\mathbb{D}_{6}$. Then by Theorem 2.10 in the neighbourhood of $O$ the singularity is isomorphic to $\mathbb{D}_{6} \times \mathbb{C}$, and by Corollary 2.7 the singularities of $V$ are canonical.

$\left(H_{40}\right):$ A double cover $V \longrightarrow \mathbb{F}(8,5,0)$. The ramification divisor $D$ lies in the linear system $|4 M-22 L|$ and is given by the form

$$
\alpha_{10} x_{1}^{4}+\alpha_{7} x_{1}^{3} x_{2}+\alpha_{2} x_{1}^{3} x_{3}+\alpha_{4} x_{1}^{2} x_{2}^{2}+\alpha_{1} x_{1} x_{2}^{3} .
$$

It is sufficient to check canonicity of the singularities of $V$ only over points of the curve $C$ given by $x_{1}=x_{2}=0$. As in Example 3.10, on the inverse image of the fiber $Y$ in the neighbourhood of the point $O=C \cap Y$ after a coordinate change $\omega=x, x_{1}=y, x_{2}=z$ and $x_{3}=1$ we have a singularity at $O$ given by the zeroes of the polynomial

$$
x^{2}+y^{4}+y^{3} z+y^{3}+y^{2} z^{2}+y z^{3} .
$$

If we put $\operatorname{wt}(x)=9, \operatorname{wt}(y)=6$ and $\operatorname{wt}(z)=4$, it's easy to check that the singularity given by the term of the lowest weight is isolated and $\mathrm{Du}$ Val. The weights coincide with the weights of $\mathbb{E}_{7}$. Then by Theorem 2.10 in the neighbourhood of $O$ the singularity is isomorphic to $\mathbb{E}_{7} \times \mathbb{C}$, and by Corollary 2.7 the singularities of $V$ are canonical.

$\left(H_{41}\right):$ A double cover $V \longrightarrow \mathbb{F}(8,6,0)$. The ramification divisor $D$ lies in the linear system $|4 M-24 L|$ and is given by the form

$$
\alpha_{8} x_{1}^{4}+\alpha_{6} x_{1}^{3} x_{2}+\alpha_{0}^{1} x_{1}^{3} x_{3}+\alpha_{4} x_{1}^{2} x_{2}^{2}+\alpha_{2} x_{1} x_{2}^{3}+\alpha_{0}^{2} x_{2}^{4} .
$$

The curve $C$ given by $x_{1}=x_{2}=0$ is the only base curve of this linear system. As in Example 3.10, on the inverse image of the fiber $Y$ in the neighbourhood of the point $O=C \cap Y$ after a coordinate change $\omega=x, x_{1}=y, x_{2}=z$ and $x_{3}=1$ we have a singularity at $O$ given by the zeroes of the polynomial

$$
x^{2}+y^{4}+y^{3} z+y^{3}+y^{2} z^{2}+y z^{3}+z^{4} .
$$

If we put $\operatorname{wt}(x)=6, \operatorname{wt}(y)=4$ and $\operatorname{wt}(z)=3$, it's easy to check that the singularity given by the term of the lowest weight is isolated and $\mathrm{Du}$ Val. The weights coincide with the weights of $\mathbb{E}_{6}$. Then by Theorem 2.10 in the neighbourhood of $O$ the singularity is isomorphic to $\mathbb{E}_{6} \times \mathbb{C}$, and by Corollary 2.7 the singularities of $V$ are canonical.

$\left(H_{42}\right)$ : A double cover $V \longrightarrow \mathbb{F}(9,5,0)$. The ramification divisor $D$ lies in the linear system $|4 M-24 L|$ and is given by the form

$$
\alpha_{12} x_{1}^{4}+\alpha_{8} x_{1}^{3} x_{2}+\alpha_{3} x_{1}^{3} x_{3}+\alpha_{4} x_{1}^{2} x_{2}^{2}+\alpha_{0} x_{1} x_{2}^{3} .
$$

It is sufficient to check canonicity of the singularities of $V$ only over points of the curve $C$ given by $x_{1}=x_{2}=0$. As in Example 3.10, on the inverse image of the fiber $Y$ in the neighbourhood of the point $O=C \cap Y$ after a coordinate change 
$\omega=x, x_{1}=y, x_{2}=z$ and $x_{3}=1$ we have a singularity at $O$ given by the zeroes of the polynomial

$$
x^{2}+y^{4}+y^{3} z+y^{3}+y^{2} z^{2}+y z^{3} .
$$

If we put $\operatorname{wt}(x)=9, \operatorname{wt}(y)=6$ and $\operatorname{wt}(z)=4$, it's easy to check that the singularity given by the term of the lowest weight is isolated and Du Val. The weights coincide with the weights of $\mathbb{E}_{7}$. Then by Theorem 2.10 in the neighbourhood of $O$ the singularity is isomorphic to $\mathbb{E}_{7} \times \mathbb{C}$, and by Corollary 2.7 the singularities of $V$ are canonical.

$\left(H_{43}\right):$ A double cover $V \longrightarrow \mathbb{F}(9,6,0)$. The ramification divisor $D$ lies in the linear system $|4 M-26 L|$ and is given by the form

$$
\alpha_{10} x_{1}^{4}+\alpha_{7} x_{1}^{3} x_{2}+\alpha_{1}^{1} x_{1}^{3} x_{3}+\alpha_{4} x_{1}^{2} x_{2}^{2}+\alpha_{1}^{2} x_{1} x_{2}^{3} .
$$

It is sufficient to check canonicity of the singularities of $V$ only over points of the curve $C$ given by $x_{1}=x_{2}=0$. As in Example 3.10, on the inverse image of the fiber $Y$ in the neighbourhood of the point $O=C \cap Y$ after a coordinate change $\omega=x, x_{1}=y, x_{2}=z$ and $x_{3}=1$ we have a singularity at $O$ given by the zeroes of the polynomial

$$
x^{2}+y^{4}+y^{3} z+y^{3}+y^{2} z^{2}+y z^{3} .
$$

If we put $\mathrm{wt}(x)=9, \operatorname{wt}(y)=6$ and $\mathrm{wt}(z)=4$, it's easy to check that the singularity given by the term of the lowest weight is isolated and $\mathrm{Du}$ Val. The weights coincide with the weights of $\mathbb{E}_{7}$. Then by Theorem 2.10 in the neighbourhood of $O$ the singularity is isomorphic to $\mathbb{E}_{7} \times \mathbb{C}$, and by Corollary 2.7 the singularities of $V$ are canonical.

$\left(H_{44}\right):$ A double cover $V \longrightarrow \mathbb{F}(10,6,0)$. The ramification divisor $D$ lies in the linear system $|4 M-28 L|$ and is given by the form

$$
\alpha_{12} x_{1}^{4}+\alpha_{8} x_{1}^{3} x_{2}+\alpha_{2} x_{1}^{3} x_{3}+\alpha_{4} x_{1}^{2} x_{2}^{2}+\alpha_{0} x_{1} x_{2}^{3} .
$$

It is sufficient to check canonicity of the singularities of $V$ only over points of the curve $C$ given by $x_{1}=x_{2}=0$. As in Example 3.10, on the inverse image of the fiber $Y$ in the neighbourhood of the point $O=C \cap Y$ after a coordinate change $\omega=x, x_{1}=y, x_{2}=z$ and $x_{3}=1$ we have a singularity at $O$ given by the zeroes of the polynomial

$$
x^{2}+y^{4}+y^{3} z+y^{3}+y^{2} z^{2}+y z^{3} .
$$

If we put $\operatorname{wt}(x)=9, \operatorname{wt}(y)=6$ and $\operatorname{wt}(z)=4$, it's easy to check that the singularity given by the term of the lowest weight is isolated and $\mathrm{Du}$ Val. The weights coincide with the weights of $\mathbb{E}_{7}$. Then by Theorem 2.10 in the neighbourhood of $O$ the singularity is isomorphic to $\mathbb{E}_{7} \times \mathbb{C}$, and by Corollary 2.7 the singularities of $V$ are canonical.

$\left(H_{45}\right):$ A double cover $V \longrightarrow \mathbb{F}(10,7,0)$. The ramification divisor $D$ lies in the linear system $|4 M-30 L|$ and is given by the form

$$
\alpha_{10} x_{1}^{4}+\alpha_{7} x_{1}^{3} x_{2}+\alpha_{0} x_{1}^{3} x_{3}+\alpha_{4} x_{1}^{2} x_{2}^{2}+\alpha_{1} x_{1} x_{2}^{3} .
$$

It is sufficient to check canonicity of the singularities of $V$ only over points of the curve $C$ given by $x_{1}=x_{2}=0$. As in Example 3.10, on the inverse image of the fiber $Y$ in the neighbourhood of the point $O=C \cap Y$ after a coordinate change 
$\omega=x, x_{1}=y, x_{2}=z$ and $x_{3}=1$ we have a singularity at $O$ given by the zeroes of the polynomial

$$
x^{2}+y^{4}+y^{3} z+y^{3}+y^{2} z^{2}+y z^{3} .
$$

If we put $\operatorname{wt}(x)=9, \operatorname{wt}(y)=6$ and $\operatorname{wt}(z)=4$, it's easy to check that the singularity given by the term of the lowest weight is isolated and Du Val. The weights coincide with the weights of $\mathbb{E}_{7}$. Then by Theorem 2.10 in the neighbourhood of $O$ the singularity is isomorphic to $\mathbb{E}_{7} \times \mathbb{C}$, and by Corollary 2.7 the singularities of $V$ are canonical.

$\left(H_{46}\right)$ : A double cover $V \longrightarrow \mathbb{F}(11,7,0)$. The ramification divisor $D$ lies in the linear system $|4 M-32 L|$ and is given by the form

$$
\alpha_{12} x_{1}^{4}+\alpha_{8} x_{1}^{3} x_{2}+\alpha_{1} x_{1}^{3} x_{3}+\alpha_{4} x_{1}^{2} x_{2}^{2}+\alpha_{0} x_{1} x_{2}^{3} .
$$

It is sufficient to check canonicity of the singularities of $V$ only over points of the curve $C$ given by $x_{1}=x_{2}=0$. As in Example 3.10, on the inverse image of the fiber $Y$ in the neighbourhood of the point $O=C \cap Y$ after a coordinate change $\omega=x, x_{1}=y, x_{2}=z$ and $x_{3}=1$ we have a singularity at $O$ given by the zeroes of the polynomial

$$
x^{2}+y^{4}+y^{3} z+y^{3}+y^{2} z^{2}+y z^{3} .
$$

If we put wt $(x)=9, \operatorname{wt}(y)=6$ and $\operatorname{wt}(z)=4$, it's easy to check that the singularity given by the term of the lowest weight is isolated and $\mathrm{Du}$ Val. The weights coincide with the weights of $\mathbb{E}_{7}$. Then by Theorem 2.10 in the neighbourhood of $O$ the singularity is isomorphic to $\mathbb{E}_{7} \times \mathbb{C}$, and by Corollary 2.7 the singularities of $V$ are canonical.

$\left(H_{47}\right):$ A double cover $V \longrightarrow \mathbb{F}(12,8,0)$. The ramification divisor $D$ lies in the linear system $|4 M-36 L|$ and is given by the form

$$
\alpha_{12} x_{1}^{4}+\alpha_{8} x_{1}^{3} x_{2}+\alpha_{0} x_{1}^{3} x_{3}+\alpha_{4} x_{1}^{2} x_{2}^{2}+\alpha_{0} x_{1} x_{2}^{3} .
$$

It is sufficient to check canonicity of the singularities of $V$ only over points of the curve $C$ given by $x_{1}=x_{2}=0$. As in Example [3.10, on the inverse image of the fiber $Y$ in the neighbourhood of the point $O=C \cap Y$ after a coordinate change $\omega=x, x_{1}=y, x_{2}=z$ and $x_{3}=1$ we have a singularity at $O$ given by the zeroes of the polynomial

$$
x^{2}+y^{4}+y^{3} z+y^{3}+y^{2} z^{2}+y z^{3} .
$$

If we put $\operatorname{wt}(x)=9, \operatorname{wt}(y)=6$ and $\operatorname{wt}(z)=4$, it's easy to check that the singularity given by the term of the lowest weight is isolated and $\mathrm{Du}$ Val. The weights coincide with the weights of $\mathbb{E}_{7}$. Then by Theorem 2.10 in the neighbourhood of $O$ the singularity is isomorphic to $\mathbb{E}_{7} \times \mathbb{C}$, and by Corollary 2.7 the singularities of $V$ are canonical.

Therefore, Theorem 1.5 is proved. Actually, the proof of Theorem 1.5 can be used to describe the singularities of every 3 -fold $H_{i}$. For example, the sufficiently general 3 -fold $H_{i}$ is smooth for $i \in\{1,2,3,4,6,9\}$. The sufficiently general 3 -fold $H_{5}$ has a single isolated ordinary double point, and it is not $\mathbb{Q}$-factorial. The singularities of the 3 -fold $H_{i}$ are non-isolated for $i \in\{8,10,12,14,16,17,18,20,22,27,29,33,38\}$. In all other cases $H_{i}$ has a single isolated non-cDV point if $H_{i}$ is general enough. 


\section{Trigonal Fano 3-Folds.}

In this section we will prove Theorem 1.6. Let $X$ be a Fano 3-fold with canonical Gorenstein singularities, such that the linear system $\left|-K_{X}\right|$ has no base points and the induced morphism $\phi_{\left|-K_{X}\right|}$ is an embedding, but the anticanonical image $\phi_{\left|-K_{X}\right|}(X) \subset \mathbb{P}^{n}$ is not an intersection of quadrics, where $n=-\frac{K_{X}^{3}}{2}+2$.

Remark 4.1. In the case when $-K_{X}^{3}=4$ the 3 -fold $X$ is a possibly singular quartic 3 -fold in $\mathbb{P}^{4}$. The birational geometry of such $X$ was studied in [67, [8], [118], [59, [96], [22], [23], [86], 17].

Remark 4.2. In the case when $-K_{X}^{3}=6$ the 3 -fold $X$ is a possibly singular complete intersection of a quadric and a cubic in $\mathbb{P}^{5}$. The latter easily follows either from Theorem 2.14 or from Proposition 2.21] The birational geometry of such Fano 3 -fold $X$ was studied in [8], 118], [59], 97], 22].

Thus, we may assume that $-K_{X}^{3} \geq 8$. Hence, Theorem 2.14 implies that $X$ is projectively normal in $\mathbb{P}^{n}$ and the quadrics in $\mathbb{P}^{n}$ passing through $X$ cut out a 4 -fold $Y \subset \mathbb{P}^{n}$ of degree $n-3$. Moreover, for a general linear subspace $\Pi \subset \mathbb{P}^{n}$ of codimension 2 the curve $X \cap \Pi$ is either a canonically embedded smooth trigonal curve or a canonically embedded smooth plane quintic curve and $\operatorname{deg}\left(X \subset \mathbb{P}^{n}\right)=10$. In the former case the 4 -fold $Y$ is an image of a rational scroll $\operatorname{Proj}\left(\oplus_{i=1}^{4} \mathcal{O}_{\mathbb{P}^{1}}\left(d_{i}\right)\right)$ via the map given by the tautological line bundle, where $0 \neq d_{1} \geq \ldots \geq d_{4} \geq 0$ and $-K_{X}^{3}=2\left(d_{1}+d_{2}+d_{3}+d_{4}\right)+2$. In the latter case $n=7$ and the 4 -fold $Y$ is a cone over a Veronese surface.

Remark 4.3. The cone in $\mathbb{P}^{7}$ over a Veronese surface is nothing but $\mathbb{P}\left(1^{3}, 2,2\right)$. Therefore, in the case when $Y$ is a cone over a Veronese surface the 3 -fold $X$ is a hypersurface in $\mathbb{P}\left(1^{3}, 2^{2}\right)$ of degree 5 , because $-K_{X}^{3}=10$.

Lemma 4.4. Let $Y$ be a cone in $\mathbb{P}^{7}$ over a Veronese surface, whose vertex is a line $L \subset \mathbb{P}^{7}$. Take a resolution of singularities $f: U \rightarrow Y$, where $U=\operatorname{Proj}\left(\mathcal{O}_{\mathbb{P}^{2}}(2) \oplus \mathcal{O}_{\mathbb{P}^{2}} \oplus \mathcal{O}_{\mathbb{P}^{2}}\right)$. Let $T=f^{*}\left(\left.\mathcal{O}_{\mathbb{P}^{7}}(1)\right|_{Y}\right)$ and $F$ be a pull back of $\mathcal{O}_{\mathbb{P}^{2}}(1)$ via the natural projection of $U$ to $\mathbb{P}^{2}$. Put $V=f^{-1}(X) \subset U$. Then $V$ has canonical Gorenstein singularities, $-K_{V}$ is big and nef, $V \sim 2 T+F$ on $U$ and $X=\phi_{\left|-r K_{V}\right|}(V)$ for $r \gg 0$. In particular, the birational morphism $\left.f\right|_{V}: V \rightarrow X$ is crepant.

Proof. The line $L$ is contained in $X$ due to Remark 4.3. On the other hand, the 3-fold $X$ must be singular along $L \subset X$. Indeed, let $O \in L$ be a point, $\Pi \subset \mathbb{P}^{7}$ be a sufficiently general linear subspace of codimension 2 passing through the point $O$ and let $C=\Pi \cap X$. Suppose that the point $O$ is smooth on the 3 -fold $X$. Then the curve $C$ is a smooth anticanonically embedded plane quintic curve. Therefore, the quadrics in $\Pi \cong \mathbb{P}^{5}$ passing through $C$ cut out a smooth Veronese surface by Theorem 2.13. On the other hand, the quadrics in $\Pi \cong \mathbb{P}^{5}$ passing through the curve $C$ cut out $Y \cap \Pi$. However, the surface $Y \cap \Pi$ must be singular, because $Y$ is singular at the point $O$ by assumption.

Therefore, the morphism $\left.f\right|_{V}: V \rightarrow X$ is crepant in the general point of the line $L \subset X$ by Proposition 2.15. The latter implies that $V$ does not contain any fiber of $f$ and $V \sim 2 T+F$ on $U$. Hence, the 3 -fold $V$ is normal (see Proposition 8.23 in [48). Therefore, $V$ has canonical Gorenstein singularities and $-K_{V}$ is a crepant pull back of $-K_{X}$.

Remark 4.5. A priori Lemma 4.4 does not imply the existence of the corresponding Fano 3-fold $X \subset Y$. However, the latter is quite easy. In the notations of Lemma 4.4 the linear system $|2 T+L|$ on the 4 -fold $U=\operatorname{Proj}\left(\mathcal{O}_{\mathbb{P}^{2}}(2) \oplus \mathcal{O}_{\mathbb{P}^{2}} \oplus \mathcal{O}_{\mathbb{P}^{2}}\right)$ is free. In particular, a 
sufficiently general divisor in the linear system $|2 T+L|$ is smooth. Let $D$ be a divisor in $|2 T+L|$ with canonical singularities. Then the adjunction formula implies $-K_{D} \sim T$. Therefore, $D$ is a weak Fano 3 -fold, i.e. the divisor $-K_{D}$ is nef and big. The vanishing theorem (see [70], [119]) implies $\phi_{\left|-K_{D}\right|}=\left.\phi_{|T|}\right|_{D}$. In particular, the 3-fold $\phi_{\left|-K_{D}\right|}(D)$ is a Fano 3-fold with canonical Gorenstein singularities.

Therefore, in the following we may assume that for a general linear subspace $\Pi \subset \mathbb{P}^{n}$ of codimension 2 the curve $X \cap \Pi$ is a canonically embedded smooth trigonal curve. Thus, the quadrics in $\mathbb{P}^{n}$ passing through the 3 -fold $X$ cut out the 4 -fold $Y$ which is an image of a rational scroll $\operatorname{Proj}\left(\oplus_{i=1}^{4} \mathcal{O}_{\mathbb{P}^{1}}\left(d_{i}\right)\right)$ via the map given by the tautological line bundle, where $0 \neq d_{1} \geq \ldots \geq d_{4} \geq 0$ and $-K_{X}^{3}=2\left(d_{1}+d_{2}+d_{3}+d_{4}\right)+2$.

Lemma 4.6. The inclusion $\operatorname{Sing}(Y) \cap X \subset \operatorname{Sing}(X)$ holds.

Proof. Let $O$ be a singular point on $Y$ such that $O \in X$ and the 3 -fold $X$ is non-singular at $O$. Take a sufficiently general linear subspace $\Pi \subset \mathbb{P}^{n}$ of codimension 2 passing through the point $O$. Put $C=\Pi \cap X$. Then the curve $C \subset \Pi \cong \mathbb{P}^{n-2}$ is a smooth anticanonically embedded trigonal curve. Therefore, the quadrics in $\Pi \cong \mathbb{P}^{5}$ passing through the curve $C$ cut out a smooth surface by Theorem 2.13. On the other hand, the quadrics in $\Pi \cong \mathbb{P}^{n-2}$ passing through the curve $C$ cut out the surface $Y \cap \Pi$ that must be singular at the point $O$, because $Y$ is singular at the point $O$ by assumption.

Let $f: U \rightarrow Y$ be a birational morphism, where $U=\operatorname{Proj}\left(\oplus_{i=1}^{3} \mathcal{O}_{\mathbb{P}^{1}}\left(d_{i}\right)\right), 0 \neq d_{1} \geq$ $\ldots \geq d_{k} \geq 0,-K_{X}^{3}=2\left(d_{1}+d_{2}+d_{3}+d_{4}\right)+2 \geq 8$ and $f=\phi_{|M|}$ for a tautological line bundle $M$ on $U$. Put $V=f^{-1}(X) \subset U$ and $h=\left.f\right|_{V}: V \rightarrow X$.

Lemma 4.7. The 3-fold $V$ has canonical Gorenstein singularities, the anticanonical divisor $-K_{V}$ is nef and big, and $K_{V} \sim h^{*}\left(K_{X}\right)$, i.e. the morphism $h$ is crepant.

Proof. The 3 -fold $V$ is normal if and only if it is smooth in codimension 1 (see Proposition 8.23 in [48]). On the other hand, in the case when $d_{3} \neq 0$ the 3 -folds $X$ and $V$ are isomorphic in codimension 2. The latter immediately implies the claim (cf. [71]).

Thus, we may assume $d_{3}=d_{4}=0$. Let $Z=\operatorname{Sing}(Y)$. Then $\operatorname{dim}(Z) \leq 2$, and the equality $\operatorname{dim}(Z)=2$ holds if and only if $d_{2}=0$. Moreover, in the case $\operatorname{dim}(Z \cap X)=0$ the 3-folds $X$ and $V$ are isomorphic in codimension 2, which implies the claim. On the other hand, $Z \cap X \subset \operatorname{Sing}(X)$ by Lemma 4.6. However, the normality of $X$ implies $\operatorname{dim}(Z \cap X) \leq \operatorname{dim}(\operatorname{Sing}(X)) \leq 1$. Thus, we may assume that the intersection $Z \cap X$ consists of a finite number of curves and $X$ is singular along every curve in $Z \cap X$.

The canonicity of the singularities of the 3 -fold $X$ and Proposition 2.15] imply that $g$ is crepant in the general point of every curve in $Z \cap X$ and the singularities of the 3-fold $V$ are canonical Gorenstein over the general point of every curve in $Z \cap X$. Thus, we proved the required claim on a complement to a subset of $V$ of codimension 2. The latter implies the normality of the 3 -fold $V$ (see Proposition 8.23 in [48]). Therefore, $V \subset U$ is a divisor on a smooth 3 -fold, which is normal and whose singularities are canonical Gorenstein in codimension 2. The latter implies that singularities of $V$ are canonical Gorenstein and $K_{V} \sim h^{*}\left(K_{X}\right)$.

Let $M$ be a tautological line bundle on $U$ and $L$ be a fiber of the natural projection of $U$ to $\mathbb{P}^{1}$. Then $-\left.K_{V} \sim M\right|_{V}$ by construction.

Remark 4.8. A priori the contraction theorem (see [2]) implies that $X$ is an anticanonical model of $V$. However, the vanishing theorem (see [70, [119]) implies that $\left|-K_{V}\right|=|M|_{V} \mid$. Therefore, a posteriori $X$ is an anticanonical image of the 3 -fold $V$, i.e. $X=\phi_{\left|-K_{V}\right|}(X)$. 
The adjunction formula implies that $V \sim 3 M-\left(d_{1}+d_{2}+d_{3}+d_{4}-2\right) L$ on $U$. Let $Y_{j} \subset V$ be a subscroll induced by a natural projections $\oplus_{i=1}^{4} \mathcal{O}_{\mathbb{P}^{1}}\left(d_{i}\right) \rightarrow \oplus_{i=j}^{4} \mathcal{O}_{\mathbb{P}^{1}}\left(d_{i}\right)$. Then $Y_{4}$ is a curve, $Y_{3}$ is a surface and $Y_{2}$ is a 3 -fold.

Lemma 4.9. The inequalities $\operatorname{mult}_{Y_{2}}(V)=0, \operatorname{mult}_{Y_{3}}(V) \leq 1$ and mult $_{Y_{4}}(V) \leq 2$ hold.

Proof. The first inequality is trivial, the second one is implied by the normality of $V$, and the last one is implied by the canonicity of the 3 -fold $V$ in a general point of $Y_{4}$ due to Theorem 2.9.

Therefore, Corollary 2.20 implies the inequalities $2 d_{2}-d_{1}-d_{3}-d_{4}+2 \geq 0, d_{3}-d_{2}-$ $d_{4}+2 \geq 0,2-d_{2}-d_{3}+d_{1} \geq 0$. Moreover, we also have $0 \neq d_{1} \geq \ldots \geq d_{4} \geq 0$ and $d_{1}+d_{2}+d_{3}+d_{4} \geq 3$ by assumption. The obtained inequalities give exactly 66 different rational scrolls $\mathbb{F}\left(d_{1}, d_{2}, d_{3}, d_{4}\right)=\operatorname{Proj}\left(\oplus_{i=1}^{4} \mathcal{O}_{\mathbb{P}^{1}}\left(d_{i}\right)\right)$.

Remark 4.10. The 3-fold $X$ is an anticanonical model of the 3 -fold $V$, i.e. $X=\phi_{\left|-r K_{V}\right|}(V)$ for $r \gg 0$. Thus, the contraction theorem (see [72]) implies that the 3-fold $X$ is a Fano 3 -fold with canonical Gorenstein singularities if and only if $V$ has canonical Gorenstein singularities and $-K_{V}$ is nef and big. On the other hand, the 3 -fold $V$ is uniquely determined by a rational scroll $\mathbb{F}\left(d_{1}, d_{2}, d_{3}, d_{4}\right)$ and a class $3 M-\left(d_{1}+d_{2}+d_{3}+d_{4}-2\right) L$ in the Picard group of $\mathbb{F}\left(d_{1}, d_{2}, d_{3}, d_{4}\right)$. However, a priori there may be no divisor in the linear system $\left|3 M-\left(d_{1}+d_{2}+d_{3}+d_{4}-2\right) L\right|$ with canonical singularities.

In the rest of the section we will explicitly show that in every obtained case there is a divisor in the linear system $\left|3 M-\left(d_{1}+d_{2}+d_{3}+d_{4}-2\right) L\right|$ with canonical singularities using Corollary 2.5 together with Proposition 2.19. The latter will conclude the proof of Theorem 1.6.

Remark 4.11. The same idea was used during the classification of smooth trigonal Fano 3 -folds (see [58, 61]) and in [14] during the effective boundness of the trigonal Fano 3 -folds with canonical singularities, which is a sharp bound due to Theorem 1.6.

First, let us consider two slightly different possible cases in full details.

Example 4.12. Let $V \subset \mathbb{F}=\mathbb{F}(6,5,3,0)$ be a sufficiently general divisor $V \in|3 M-12 L|$. Let us show that $V$ has canonical singularities.

By Proposition 2.19 the divisor $V$ is given by the zeroes of a bihomogeneous polynomial

$$
\begin{aligned}
\alpha_{6}\left(t_{1}, t_{2}\right) x_{1}^{3}+\alpha_{5}\left(t_{1}, t_{2}\right) & x_{1}^{2} x_{2}+\alpha_{3}^{1}\left(t_{1}, t_{2}\right) x_{1}^{2} x_{3}+\alpha_{0}^{1}\left(t_{1}, t_{2}\right) x_{1}^{2} x_{4}+\alpha_{4}\left(t_{1}, t_{2}\right) x_{1} x_{2}^{2}+ \\
+ & \alpha_{2}\left(t_{1}, t_{2}\right) x_{1} x_{2} x_{3}+\alpha_{0}^{2}\left(t_{1}, t_{2}\right) x_{1} x_{3}^{2}+\alpha_{3}^{2}\left(t_{1}, t_{2}\right) x_{2}^{3}+\alpha_{1}\left(t_{1}, t_{2}\right) x_{2}^{2} x_{3},
\end{aligned}
$$

where $\alpha_{d}\left(t_{1}, t_{2}\right)$ (or $\alpha_{d}^{i}\left(t_{1}, t_{2}\right)$ ) is a form of degree $d$. Let $E$ be a surface given by $x_{1}=x_{2}=0$ and $C$ be a curve given by $x_{1}=x_{2}=x_{3}=0$. Note that the base locus of $|3 M-12 L|$ is exactly $E$, but as the automorphism group of $E \cong \mathbb{F}(3,0)$ acts transitively on $E \backslash C$ and $V$ has multiplicity 1 at a general point of $E$, by Corollary 2.5] it is sufficient to prove that for any point $P$ of $C$ there exists a divisor $V$ with canonical singularities in neighbourhood of the point $P$.

Let $Y$ be a fiber of $V \subset \mathbb{F}$ over a sufficiently general point $P \in \mathbb{P}^{1}$. Let $O=C \cap Y$. According to the above it is sufficient to prove that $V$ has only canonical singularities in the neighbourhood of the point $O$, and for the latter it is sufficient to prove that $O$ is a Du Val point on $Y$. 
Let the homogeneous coordinates of the point $P \in \mathbb{P}^{1}$ be $(\gamma: \delta)$. Then $Y$ can be given as a hypersurface

$$
\alpha_{6} x_{1}^{3}+\alpha_{5} x_{1}^{2} x_{2}+\alpha_{3}^{1} x_{1}^{2} x_{3}+\alpha_{0}^{1} x_{1}^{2} x_{4}+\alpha_{4} x_{1} x_{2}^{2}+\alpha_{2} x_{1} x_{2} x_{3}+\alpha_{0}^{2} x_{1} x_{3}^{2}+\alpha_{3}^{2} x_{2}^{3}+\alpha_{1} x_{2}^{2} x_{3}=0
$$

in $\mathbb{P}^{4}$, where $\alpha_{d}=\alpha_{d}(\gamma, \delta)$ and $O=(0: 0: 0: 1)$. Since $P$ is general we have $\alpha_{i} \neq 0$ for all $i$. Therefore, for convenience we may put $\alpha_{i}^{j}=1$ for all $i$ and $j$.

Let $x_{1}=x, x_{2}=y, x_{3}=z$ and $x_{4}=1$. Then the local equation of $Y$ in the neighbourhood of $O$ is

$$
x^{3}+x^{2} y+x^{2} z+x^{2}+x y^{2}+x y z+x z^{2}+y^{3}+y^{2} z=0 .
$$

Let $\operatorname{wt}(x)=4, \operatorname{wt}(y)=3$ and $\operatorname{wt}(z)=2$. Then $\operatorname{wt}\left(x^{2}+x z^{2}+y^{2} z\right)=8$ and $\operatorname{wt}\left(x^{3}\right)=12$, $\operatorname{wt}\left(x^{2} y\right)=11, \operatorname{wt}\left(x y^{2}\right)=10, \operatorname{wt}\left(y^{3}\right)=9, \operatorname{wt}\left(x^{2} z\right)=10$ and $\operatorname{wt}(x y z)=9$. Moreover, the singularity given by the equation $x^{2}+x z^{2}+y^{2} z=0$ is isolated. Therefore, the singularity of the surface $Y$ at the point $O$ is locally isomorphic to a Du Val singularity of type $\mathbb{D}_{5}$ by Theorem 2.10. In particular, at a general point of $C$ the 3-fold $V$ has a singularity of type $\mathbb{D}_{5} \times \mathbb{C}$. Actually, we may assume that $P$ is an arbitrary point on the curve $C$ due to the generality in the choice of $V$ (cf. Example 3.10). Thus, for any given point $P$ on the curve $C$ there is a divisor in $|3 M-12 L|$ with at most canonical singularities in the neighbourhood of $P$. Hence, the singularities of $V$ are canonical by Corollary 2.5.

Example 4.13. Let $V \subset \mathbb{F}=\mathbb{F}(7,3,1,0)$ be a general divisor in the linear system $|3 M-9 L|$. Let us show that $V$ has canonical singularities.

By Proposition 2.19 the divisor $V$ is given by the zeroes of a bihomogeneous polynomial

$$
\begin{gathered}
\alpha_{12}\left(t_{1}, t_{2}\right) x_{1}^{3}+\alpha_{8}\left(t_{1}, t_{2}\right) x_{1}^{2} x_{2}+\alpha_{6}\left(t_{1}, t_{2}\right) x_{1}^{2} x_{3}+\alpha_{5}\left(t_{1}, t_{2}\right) x_{1}^{2} x_{4}+ \\
+\alpha_{4}\left(t_{1}, t_{2}\right) x_{1} x_{2}^{2}+\alpha_{2}\left(t_{1}, t_{2}\right) x_{1} x_{2} x_{3}+\alpha_{1}\left(t_{1}, t_{2}\right) x_{1} x_{2} x_{4}+\alpha_{0}^{1}\left(t_{1}, t_{2}\right) x_{1} x_{3}^{2}+\alpha_{0}^{2}\left(t_{1}, t_{2}\right) x_{2}^{3},
\end{gathered}
$$

where $\alpha_{d}\left(t_{1}, t_{2}\right)$ (or $\left.\alpha_{d}^{i}\left(t_{1}, t_{2}\right)\right)$ is a form of degree $d$. Let $E$ be a surface given by $x_{1}=x_{2}=0$ and $C$ be a curve given by $x_{1}=x_{2}=x_{3}=0$. Note that the base locus of $|3 M-9 L|$ is exactly $E$, but as the automorphism group of $E \cong \mathbb{F}(1,0)$ acts transitively on $E \backslash C$ and $V$ has multiplicity 1 at a general point of $E$, by Corollary 2.5] it is sufficient to prove that for any point $P$ of $C$ there exists a divisor $V$ with canonical singularities at $P$.

Let $Y$ be a fiber of $V \subset \mathbb{F}$ over a general point $P \in \mathbb{P}^{1}$. Let $O=C \cap Y$. According to the above it is sufficient to prove that $V$ has only canonical singularities at $O$, and for the latter it is sufficient to prove that $O$ is a Du Val point on $Y$.

Let the homogeneous coordinates of the point $P \in \mathbb{P}^{1}$ be $(\gamma: \delta)$. Then $Y$ can be given as a hypersurface corresponding to the following polynomial

$$
\alpha_{12} x_{1}^{3}+\alpha_{8} x_{1}^{2} x_{2}+\alpha_{6} x_{1}^{2} x_{3}+\alpha_{5} x_{1}^{2} x_{4}+\alpha_{4} x_{1} x_{2}^{2}+\alpha_{2} x_{1} x_{2} x_{3}++\alpha_{1} x_{1} x_{2} x_{4}+\alpha_{0}^{1} x_{1} x_{3}^{2}+\alpha_{0}^{2} x_{2}^{3}
$$

in $\mathbb{P}^{4}$, where $\alpha_{d}=\alpha_{d}(\gamma, \delta)$ and $O=(0: 0: 0: 1)$. Since $P$ is general we have $\alpha_{i} \neq 0$ for all $i$. Therefore, for convenience we may put $\alpha_{i}^{j}=1$ for all $i$ and $j$.

Let $x_{1}=x, x_{2}=y, x_{3}=z$ and $x_{4}=1$. Then the local equation of $Y$ in the neighbourhood of $O$ is

$$
x^{3}+x^{2} y+x^{2} z+x^{2}+x y^{2}+x y z+x y+x z^{2}+y^{3}=0 .
$$

It's easy to prove that we can not choose "good" weights, so we can not use Theorem 2.10 as before. So we should resolve the only singularity in $O$. It is easy to see that for resolution of $O$ we can skip the monomials whose weight is larger than at least one of 
the others. The latter will be clear from the forthcoming blow ups. So it is sufficient to consider only the following polynomial

$$
x^{2}+x y+x z^{2}+y^{3} .
$$

First of all, the singularity at $O$ given by this polynomial is isolated. Let us blow up this point. In the local maps we will have:

- $x \neq 0$. After coordinate change $x=x, y=x y, z=x z$ we will have (after dividing by $x^{2}$ ) the local equation

$$
1+y+x z^{2}+x y^{3}=0
$$

so in this map our surface is smooth.

- $y \neq 0$. After coordinate change $x=x y, y=y, z=y z$ we will have (after dividing by $y^{2}$ ) local equation

$$
x^{2}+x+x y z^{2}+y=0,
$$

so in this map our surface is smooth.

- $z \neq 0$. After coordinate change $x=x z, y=y z, z=z$ we will have (after dividing by $z^{2}$ ) the local equation

$$
x^{2}+x y+x z+y^{3} z=0 .
$$

So we have two extremal $(-2)$-curves with only one singular point $(0,0,0)$ near $z=0$.

Therefore, we didn't resolve the original singularity yet. Hence, we must study the singularity given by the local equation

$$
x^{2}+x y+x z+y^{3} z=0 .
$$

Let us blow up the point $(0,0,0)$.

- $x \neq 0$. After coordinate change $x=x, y=x y, z=x z$ we will have (after dividing by $x^{2}$ ) the local equation

$$
1+y+z+x^{2} y^{3} z=0
$$

so in this map our surface is smooth.

- $y \neq 0$. After coordinate change $x=x y, y=y, z=y z$ we will have (after dividing by $y^{2}$ ) the defining polynomial

$$
x^{2}+x+x z+y^{2} z=x^{2}+x(z+1)+y^{2}(z+1)-y^{2}=x^{2}+y^{\prime 2}+z^{\prime 2}-y^{\prime 2} z^{\prime},
$$

where $y^{\prime}=i y$ and $z^{\prime}=z+1$. Thus, the point $(0,0,-1)$ is Du Val of type $\mathbb{A}_{1}$.

- $z \neq 0$. After coordinate change $x=x z, y=y z, z=z$ we will have (after dividing by $z^{2}$ ) the local equation

$$
x^{2}+x y+x+y^{3} z^{2}=0 .
$$

We can see that the point $(0,-1,0)$ is the singular, but it is the same point as in the singular point in the map $y \neq 0$.

Summarizing, we have two (-2)-curves after the first blow up, two (-2)-curves after the second and one point of type $\mathbb{A}_{1}$ on one of them. Hence, the graph of the resolution of the original singularity correpsonds to a Du Val singularity of type $\mathbb{A}_{5}$. In particular, at the general point of $C$ the 3 -fold $V$ has a singularity of type $\mathbb{A}_{5} \times \mathbb{C}$. Hence, as in the Example 4.12 the singularities of $V$ are canonical by Corollary 2.5 . 
In the rest of the section we will consider all the possible 66 cases following either the pattern of Example 4.12 or the pattern of Example 4.13 using the similar notations but omitting the unnecessary details. We will label every case by the corresponding name of $X$ used in the statement of Theorem [1.6, i.e. $T_{4}, T_{5}, \ldots, T_{69}$.

$\left(T_{4}\right)$ : A divisor $V \subset \mathbb{F}(1,1,1,0)$ from the linear system $|3 M-L|$. It's easy to check that $V$ is given by the form

$$
\begin{aligned}
& \alpha_{2}^{1} x_{1}^{3}+\alpha_{2}^{2} x_{1}^{2} x_{2}+\alpha_{2}^{3} x_{1}^{2} x_{3}+\alpha_{2}^{4} x_{1}^{2} x_{2}+\alpha_{2}^{5} x_{1} x_{2} x_{3}+\alpha_{2}^{6} x_{1} x_{3}^{2}+\alpha_{2}^{7} x_{2}^{3}+ \\
& \quad+\alpha_{2}^{8} x_{2}^{2} x_{3}+\alpha_{2}^{9} x_{2} x_{3}^{2}+\alpha_{2}^{10} x_{3}^{3}+\alpha_{1}^{1} x_{1}^{2} x_{4}+\alpha_{1}^{2} x_{1} x_{2} x_{4}+ \\
& +\alpha_{1}^{3} x_{1} x_{3} x_{4}+\alpha_{1}^{4} x_{2}^{2} x_{4}+\alpha_{1}^{5} x_{2} x_{3} x_{4}+\alpha_{1}^{5} x_{3}^{2} x_{4}+\alpha_{0}^{1} x_{1} x_{4}^{2}+\alpha_{0}^{2} x_{2} x_{4}^{2}+\alpha_{0}^{3} x_{3} x_{4}^{2} .
\end{aligned}
$$

After a coordinate change similar to one considered in Example 4.12 a (possible) singularity at $O$ is given by the zeroes of the polynomial

$$
P_{1}(x, y, z)+P_{2}(x, y, z)+P_{3}(x, y, z),
$$

where $P_{i}$ is a general homogeneous polynomial of degree $i$, and so $O$ is a smooth point. By Corollary 2.5 the singularities of $V$ are canonical. Therefore, the 3 -fold $X \subset \mathbb{P}^{6}$ is an anticanonically Fano 3 -fold with canonical Gorenstein singularities and $-K_{X}^{3}=8$, which is not an intersection of quadrics passing through $X$.

$\left(T_{5}\right)$ : A divisor $V \subset \mathbb{F}(1,1,1,1)$ from the linear system $|3 M-2 L|$. Since $|3 M-2 L|$ is base point free, $V$ may be chosen smooth.

$\left(T_{6}\right)$ : A divisor $V \subset \mathbb{F}(2,1,0,0)$ from the linear system $|3 M-L|$. It's easy to check that $V$ is given by the form

$$
\begin{array}{r}
\alpha_{5} x_{1}^{3}+\alpha_{4} x_{1}^{2} x_{2}+\alpha_{3}^{1} x_{1}^{2} x_{3}+\alpha_{3}^{2} x_{1}^{2} x_{4}+\alpha_{3}^{3} x_{1} x_{2}^{2}+\alpha_{2}^{1} x_{1} x_{2} x_{3}+\alpha_{2}^{2} x_{1} x_{2} x_{4}+\alpha_{1}^{1} x_{1} x_{3}^{2}+ \\
+\alpha_{1}^{2} x_{1} x_{3} x_{4}+\alpha_{1}^{3} x_{1} x_{4}^{2}+\alpha_{2}^{3} x_{2}^{3}+\alpha_{1}^{4} x_{2}^{2} x_{3}+\alpha_{1}^{5} x_{2}^{2} x_{4}+\alpha_{0}^{1} x_{2} x_{3}^{2}+\alpha_{0}^{2} x_{2} x_{3} x_{4}+\alpha_{0}^{3} x_{2} x_{4}^{2} .
\end{array}
$$

After a coordinate change similar to one considered in Example 4.12 the singularity at $O$ is given by the zeroes of the polynomial

$$
\begin{aligned}
x^{3}+x^{2} y+x^{2} z+x^{2}+x y^{2}+ & x y z+x y+ \\
& +x z^{2}+x z+x+y^{3}+y^{2} z+y^{2}+y z^{2}+y z+y .
\end{aligned}
$$

It's easy to see that $V$ may be chosen smooth at the points of $C$, and so by Corollary 2.5 the singularities of $V$ are canonical. Therefore, the 3 -fold $X \subset \mathbb{P}^{6}$ is an anticanonically Fano 3-fold with canonical Gorenstein singularities and $-K_{X}^{3}=$ 8, which is not an intersection of quadrics passing through $X$.

$\left(T_{7}\right)$ : A divisor $V \subset \mathbb{F}(2,1,1,0)$ from the linear system $|3 M-2 L|$. It's easy to check that $V$ is given by the form

$$
\begin{aligned}
\alpha_{4} x_{1}^{3} & +\alpha_{3}^{1} x_{1}^{2} x_{2}+\alpha_{3}^{2} x_{1}^{2} x_{3}+\alpha_{2}^{1} x_{1}^{2} x_{4}+\alpha_{2}^{2} x_{1} x_{2}^{2}+\alpha_{2}^{3} x_{1} x_{2} x_{3}+\alpha_{1}^{1} x_{1} x_{2} x_{4}+\alpha_{2}^{4} x_{1} x_{3}^{2}+\alpha_{1}^{2} x_{1} x_{3} x_{4}+ \\
& +\alpha_{0}^{1} x_{1} x_{4}^{2}+\alpha_{1}^{3} x_{2}^{3}+\alpha_{1}^{4} x_{2}^{2} x_{3}+\alpha_{0}^{2} x_{2}^{2} x_{4}+\alpha_{1}^{5} x_{2} x_{3}^{2}+\alpha_{0}^{3} x_{2} x_{3} x_{4}+\alpha_{1}^{6} x_{3}^{3}+\alpha_{0}^{4} x_{3}^{2} x_{4} .
\end{aligned}
$$

After a coordinate change similar to one considered in Example 4.12 the singularity at $O$ is given by the zeroes of the polynomial

$$
\begin{aligned}
x^{3}+x^{2} y+x^{2} z+x^{2}+x y^{2}+ & x y z+x y+x z^{2}+ \\
& +x z+x+y^{3}+y^{2} z+y^{2}+y z^{2}+y z+z^{3}+z^{2} .
\end{aligned}
$$


It's easy to see that $V$ may be chosen smooth at a general point of $C$, and so by Corollary 2.5 the singularities of $V$ are canonical. Note, that linear system $|M-L|$ induces a birational map $\psi: V \rightarrow \mathbb{P}^{3}$ that can be factorize as follows $\psi=\omega \circ \gamma \circ \beta$, where $\beta$ is a flop in the curve $C, \gamma$ is a contraction of a proper transform of the surface cut on $V$ by $x_{1}=0$ to a smooth rational curve whose image on $\mathbb{P}^{3}$ is a line, $\omega$ is a double cover of $\mathbb{P}^{3}$ ramified in a smooth quartic surface. Therefore, $V$ is birational to a hypersurface in $\mathbb{P}\left(1^{4}, 2\right)$ of degree 4 , so-called double quartic solid of a double space of index 2 (see [8], 112, [113, [118, 114, [19], 115]).

$\left(T_{8}\right)$ : A divisor $V \subset \mathbb{F}(2,1,1,1)$ from the linear system $|3 M-3 L|$. Since $|3 M-3 L|$ is base point free, $V$ may be chosen smooth.

$\left(T_{9}\right)$ : A divisor $V \subset \mathbb{F}(2,2,0,0)$ from the linear system $|3 M-2 L|$. It's easy to check that $V$ is given by the form

$$
\begin{aligned}
\alpha_{4}^{1} x_{1}^{3}+ & \alpha_{4}^{2} x_{1}^{2} x_{2}+ \\
+ & \alpha_{2}^{1} x_{1}^{2} x_{3}+\alpha_{2}^{2} x_{1}^{2} x_{4}+\alpha_{4}^{3} x_{1} x_{2}^{2}+ \\
+ & \alpha_{2}^{3} x_{1} x_{2} x_{3}+\alpha_{2}^{4} x_{1} x_{2} x_{4}+\alpha_{0}^{1} x_{1} x_{3}^{2}+\alpha_{0}^{2} x_{1} x_{3} x_{4}+\alpha_{0}^{3} x_{1} x_{4}^{2}+ \\
& +\alpha_{4}^{4} x_{2}^{3}+\alpha_{2}^{5} x_{2}^{2} x_{3}+\alpha_{2}^{6} x_{2}^{2} x_{4}+\alpha_{0}^{4} x_{2} x_{3}^{2}+\alpha_{0}^{5} x_{2} x_{3} x_{4}+\alpha_{0}^{6} x_{2} x_{4}^{2} .
\end{aligned}
$$

After a coordinate change similar to one considered in Example 4.12 the singularity at $O$ is given by the zeroes of the polynomial

$$
\begin{aligned}
x^{3}+x^{2} y+x^{2} z+x^{2}+x y^{2}+x y z+x y+x z^{2} & +x z+x+ \\
& +y^{3}+y^{2} z+y^{2}+y z^{2}+y z+y .
\end{aligned}
$$

It's easy to see that $V$ may be chosen smooth at the points of $C$, and so by Corollary 2.5 the singularities of $V$ are canonical.

$\left(T_{10}\right)$ : A divisor $V \subset \mathbb{F}(2,2,1,0)$ from the linear system $|3 M-3 L|$. It's easy to check that $V$ is given by the form

$$
\begin{aligned}
& \alpha_{3}^{1} x_{1}^{3}+\alpha_{3}^{2} x_{1}^{2} x_{2}+\alpha_{2}^{1} x_{1}^{2} x_{3}+\alpha_{1}^{1} x_{1}^{2} x_{4}+\alpha_{3}^{3} x_{1} x_{2}^{2}+ \\
& +\alpha_{2}^{2} x_{1} x_{2} x_{3}+\alpha_{1}^{2} x_{1} x_{2} x_{4}+\alpha_{1}^{3} x_{1} x_{3}^{2}+\alpha_{0}^{1} x_{1} x_{3} x_{4}+\alpha_{3}^{4} x_{2}^{3}+ \\
& \quad+\alpha_{2}^{3} x_{2}^{2} x_{3}+\alpha_{1}^{4} x_{2}^{2} x_{4}+\alpha_{1}^{5} x_{2} x_{3}^{2}+\alpha_{0}^{2} x_{2} x_{3} x_{4}+\alpha_{0}^{3} x_{3}^{3} .
\end{aligned}
$$

After a coordinate change similar to one considered in Example 4.12 the singularity at $O$ is given by the zeroes of the polynomial

$$
P_{3}(x, y)+P_{2}^{1}(x, y) z+P_{2}^{2}(x, y)+P_{1}(x, y) z,
$$

where $P_{i}$ (or $P_{i}^{j}$ ) is a general homogeneous polynomial of degree $i$. If we put $\operatorname{wt}(x)=\operatorname{wt}(y)=\operatorname{wt}(z)=1$, it's easy to check that the term of the lowest weight defines a singularity that is isolated. The weights coincide with the weights of $\mathbb{A}_{1}$. Then by Theorem 2.10 in the neighbourhood of $O$ the singularity is isomorphic to $\mathbb{A}_{1} \times \mathbb{C}$, and by Corollary 2.5 the singularities of $V$ are canonical. 
( $\left.T_{11}\right)$ : A divisor $V \subset \mathbb{F}(2,2,1,1)$ from the linear system $|3 M-4 L|$. It's easy to check that $V$ is given by the form

$$
\begin{aligned}
\alpha_{2}^{1} x_{1}^{3}+\alpha_{2}^{2} x_{1}^{2} x_{2}+\alpha_{1}^{1} x_{1}^{2} x_{3}+\alpha_{1}^{2} x_{1}^{2} x_{4}+ & \\
+ & \alpha_{2}^{3} x_{1} x_{2}^{2}+\alpha_{1}^{3} x_{1} x_{2} x_{3}+\alpha_{1}^{4} x_{1} x_{2} x_{4}+\alpha_{0}^{1} x_{1} x_{3}^{2}+ \\
+ & \alpha_{0}^{2} x_{1} x_{3} x_{4}+\alpha_{0}^{3} x_{1} x_{4}^{2}+\alpha_{2}^{4} x_{2}^{3}+\alpha_{1}^{5} x_{2}^{2} x_{3}+ \\
& +\alpha_{1}^{6} x_{2}^{2} x_{4}+\alpha_{0}^{4} x_{2} x_{3}^{2}+\alpha_{0}^{5} x_{2} x_{3} x_{4}+\alpha_{0}^{6} x_{2} x_{4}^{2} .
\end{aligned}
$$

After a coordinate change similar to one considered in Example 4.12 the singularity at $O$ is given by the zeroes of the polynomial

$$
\begin{aligned}
x^{3}+x^{2} y+x^{2} z+x^{2}+x y^{2}+x y z+x y+x z^{2}+x z+x+ \\
y^{3}+y^{2} z+y^{2}+y z^{2}+y z+y,
\end{aligned}
$$

It's easy to see that $V$ may be chosen smooth at the points of $C$, and so by Corollary 2.5 the singularities of $V$ are canonical.

$\left(T_{12}\right)$ : A divisor $V \subset \mathbb{F}(2,2,2,0)$ from the linear system $|3 M-4 L|$. It's easy to check that $V$ is given by the form

$$
\begin{aligned}
& \alpha_{2}^{1} x_{1}^{3}+\alpha_{2}^{2} x_{1}^{2} x_{2}+\alpha_{2}^{3} x_{1}^{2} x_{3}+\alpha_{0}^{1} x_{1}^{2} x_{4}+\alpha_{2}^{4} x_{1} x_{2}^{2}+\alpha_{2}^{5} x_{1} x_{2} x_{3}+\alpha_{0}^{2} x_{1} x_{2} x_{4}+\alpha_{2}^{6} x_{1} x_{3}^{2}+ \\
+ & \alpha_{0}^{3} x_{1} x_{3} x_{4}+\alpha_{2}^{7} x_{2}^{3}+\alpha_{2}^{8} x_{2}^{2} x_{3}+\alpha_{0}^{4} x_{2}^{2} x_{4}+\alpha_{2}^{9} x_{2} x_{3}^{2}+\alpha_{0}^{5} x_{2} x_{3} x_{4}+\alpha_{2}^{10} x_{3}^{3}+\alpha_{0}^{6} x_{3}^{2} x_{4} .
\end{aligned}
$$

After a coordinate change similar to one considered in Example 4.12 the singularity at $O$ is given by the zeroes of the polynomial

$$
P_{3}(x, y, z)+P_{2}(x, y, z),
$$

where $P_{i}$ is a general homogeneous polynomial of degree $i$. If we put $\operatorname{wt}(x)=$ $\operatorname{wt}(y)=\operatorname{wt}(z)=1$, it's easy to check that the term of the lowest weight defines a singularity that is isolated. The weights coincide with the weights of $\mathbb{A}_{1}$. Then by Theorem 2.10] in the neighbourhood of $O$ the singularity is isomorphic to $\mathbb{A}_{1} \times \mathbb{C}$, and by Corollary 2.5 the singularities of $V$ are canonical.

$\left(T_{13}\right)$ : A divisor $V \subset \mathbb{F}(2,2,2,1)$ from the linear system $|3 M-5 L|$. It's easy to check that $V$ is given by the form

$$
\begin{aligned}
\alpha_{1}^{1} x_{1}^{3}+\alpha_{1}^{2} x_{1}^{2} x_{2} & +\alpha_{1}^{3} x_{1}^{2} x_{3}+\alpha_{0}^{1} x_{1}^{2} x_{4}+ \\
+ & \alpha_{1}^{4} x_{1} x_{2}^{2}+\alpha_{1}^{5} x_{1} x_{2} x_{3}+\alpha_{0}^{2} x_{1} x_{2} x_{4}+\alpha_{1}^{6} x_{1} x_{3}^{2}+ \\
+ & \alpha_{0}^{3} x_{1} x_{3} x_{4}+\alpha_{1}^{7} x_{2}^{3}+ \\
& \alpha_{1}^{8} x_{2}^{2} x_{3}+\alpha_{0}^{4} x_{2}^{2} x_{4}+ \\
& +\alpha_{1}^{9} x_{2} x_{3}^{2}+\alpha_{0}^{5} x_{2} x_{3} x_{4}+\alpha_{1}^{10} x_{3}^{3}+\alpha_{0}^{6} x_{3}^{2} x_{4} .
\end{aligned}
$$

After a coordinate change similar to one considered in Example 4.12 the singularity at $O$ is given by the zeroes of the polynomial

$$
P_{3}(x, y, z)+P_{2}(x, y, z),
$$

where $P_{i}$ is a general homogeneous polynomial of degree $i$. If we put $\operatorname{wt}(x)=$ $\operatorname{wt}(y)=\operatorname{wt}(z)=1$, it's easy to check that the term of the lowest weight defines a singularity that is isolated. The weights coincide with the weights of $\mathbb{A}_{1}$. Then by Theorem 2.10 in the neighbourhood of $O$ the singularity is isomorphic to $\mathbb{A}_{1} \times \mathbb{C}$, and by Corollary 2.5 the singularities of $V$ are canonical. 
$\left(T_{14}\right)$ : A divisor $V \subset \mathbb{F}(2,2,2,2)$ from the linear system $|3 M-6 L|$. Since $|3 M-6 L|$ is base point free, $V$ may be chosen smooth.

$\left(T_{15}\right)$ : A divisor $V \subset \mathbb{F}(3,1,0,0)$ from the linear system $|3 M-2 L|$. Then $V$ is given by the form

$$
\begin{aligned}
\alpha_{7} x_{1}^{3} & +\alpha_{5} x_{1}^{2} x_{2}+\alpha_{4}^{1} x_{1}^{2} x_{3}+\alpha_{4}^{2} x_{1}^{2} x_{4}+\alpha_{3} x_{1} x_{2}^{2}+\alpha_{2}^{1} x_{1} x_{2} x_{3}+ \\
& +\alpha_{2}^{2} x_{1} x_{2} x_{4}+\alpha_{1}^{1} x_{1} x_{3}^{2}+\alpha_{1}^{2} x_{1} x_{3} x_{4}+\alpha_{1}^{3} x_{1} x_{4}^{2}+\alpha_{1}^{4} x_{2}^{3}+\alpha_{0}^{1} x_{2}^{2} x_{3}+\alpha_{0}^{2} x_{2}^{2} x_{4} .
\end{aligned}
$$

After a coordinate change similar to one considered in Example 4.12 the singularity at $O$ is given by the zeroes of the polynomial

$$
x^{3}+x^{2} y+x^{2} z+x^{2}+x y^{2}+x y z+x y+x z^{2}+x z+x+y^{3}+y^{2} z+y^{2} .
$$

Therefore, the 3 -fold $V$ may be chosen smooth at a general point $P \in C$, and so by Corollary 2.5 the singularities of $V$ are canonical. However, the 3 -fold $V$ is singular along the possible reducible curve $x_{1}=x_{2}=\alpha_{1}^{1} x_{3}^{2}+\alpha_{1}^{2} x_{3} x_{4}+\alpha_{1}^{3} x_{4}^{2}=0$.

$\left(T_{16}\right)$ : A divisor $V \subset \mathbb{F}(3,1,1,0)$ from the linear system $|3 M-3 L|$. It's easy to check that $V$ is given by the form

$$
\begin{aligned}
\alpha_{6} x_{1}^{3}+\alpha_{4}^{1} x_{1}^{2} x_{2}+\alpha_{4}^{2} x_{1}^{2} x_{3}+\alpha_{3} x_{1}^{2} x_{4} & +\alpha_{2}^{1} x_{1} x_{2}^{2}+ \\
+\alpha_{2}^{2} x_{1} x_{2} x_{3}+\alpha_{1}^{1} x_{1} x_{2} x_{4} & +\alpha_{2}^{3} x_{1} x_{3}^{2}+\alpha_{1}^{2} x_{1} x_{3} x_{4}+ \\
& +\alpha_{0}^{1} x_{1} x_{4}^{2}+\alpha_{0}^{2} x_{2}^{3}+\alpha_{0}^{3} x_{2}^{2} x_{3}+\alpha_{0}^{4} x_{2} x_{3}^{2}+\alpha_{0}^{5} x_{3}^{3} .
\end{aligned}
$$

After a coordinate change similar to one considered in Example 4.12 a (possible) singularity at $O$ is given by the zeroes of the polynomial

$$
x Q_{1}(x, y, z)+Q_{2}(y, z),
$$

where $Q_{1}$ is a polynomial with nonzero constant term and $Q_{2}$ is a homogeneous polynomial of degree 3. So $V$ is smooth at a general point of $C$, and by Corollary 2.5 singularities of $V$ are canonical.

$\left(T_{17}\right)$ : A divisor $V \subset \mathbb{F}(3,2,0,0)$ from the linear system $|3 M-3 L|$. It is very easy to check that $V$ is given by the form

$$
\begin{aligned}
\alpha_{6} x_{1}^{3}+\alpha_{5} x_{1}^{2} x_{2}+ & \alpha_{3}^{1} x_{1}^{2} x_{3}+\alpha_{3}^{2} x_{1}^{2} x_{4}+\alpha_{4} x_{1} x_{2}^{2}+\alpha_{2}^{1} x_{1} x_{2} x_{3}+\alpha_{2}^{2} x_{1} x_{2} x_{4}+ \\
& +\alpha_{0}^{1} x_{1} x_{3}^{2}+\alpha_{0}^{2} x_{1} x_{3} x_{4}+\alpha_{0}^{3} x_{1} x_{4}^{2}+\alpha_{3}^{3} x_{2}^{3}+\alpha_{1}^{1} x_{2}^{2} x_{3}+\alpha_{1}^{2} x_{2}^{2} x_{4} .
\end{aligned}
$$

After a coordinate change similar to one considered in Example 4.12 a (possible) singularity at $O$ is given by the zeroes of the polynomial

$$
x Q_{1}(x, y, z)+y^{2} Q_{2}(y, z),
$$

where $Q_{1}$ is a polynomial with nonzero constant term and $Q_{2}$ is a polynomial of degree 1 . So $V$ is smooth at a general point of $C$, and by Corollary 2.5. singularities of $V$ are canonical. On the other hand, the 3 -fold $V$ is always singular along the curves given by bihomogeneous equations $x_{1}=x_{2}=\alpha_{0}^{1} x_{3}^{2}+\alpha_{0}^{2} x_{3} x_{4}+\alpha_{0}^{3} x_{4}^{2}=0$, which are the sections of the natural projection of the scroll $\mathbb{F}(3,2,0,0)$ to $\mathbb{P}^{1}$. 
$\left(T_{18}\right)$ : A divisor $V \subset \mathbb{F}(3,2,1,0)$ from the linear system $|3 M-4 L|$. It's easy to check that $V$ is given by the form

$$
\begin{aligned}
\alpha_{5} x_{1}^{3}+\alpha_{4} x_{1}^{2} x_{2}+ & \alpha_{3}^{1} x_{1}^{2} x_{3}+\alpha_{2}^{1} x_{1}^{2} x_{4}+\alpha_{3}^{2} x_{1} x_{2}^{2}+\alpha_{2}^{2} x_{1} x_{2} x_{3}+\alpha_{1}^{1} x_{1} x_{2} x_{4}+ \\
& +\alpha_{1}^{2} x_{1} x_{3}^{2}+\alpha_{0}^{1} x_{1} x_{3} x_{4}+\alpha_{2}^{3} x_{2}^{3}+\alpha_{1}^{3} x_{2}^{2} x_{3}+\alpha_{0}^{2} x_{2}^{2} x_{4}+\alpha_{0}^{3} x_{2} x_{3}^{2} .
\end{aligned}
$$

After a coordinate change similar to one considered in Example 4.12 the singularity at $O$ is given by the zeroes of the polynomial

$$
x^{2} Q_{1}(x, y, z)+x y Q_{2}(x, y, z)+x z Q_{3}(x, y, z)+y^{2} Q_{4}(x, y, z)+y z^{2},
$$

where $Q_{i}$ is a polynomial with nonzero constant term. If we put $\operatorname{wt}(x)=1$, $\operatorname{wt}(y)=1$ and $\operatorname{wt}(z)=1$, it's easy to check that the singularity given by the term of the lowest weight is isolated. The weights coincide with the weights of $\mathbb{A}_{1}$. Then by Theorem 2.10 in the neighbourhood of $O$ the singularity is isomorphic to $\mathbb{A}_{1} \times \mathbb{C}$, and by Corollary 2.5 the singularities of $V$ are canonical.

$\left(T_{19}\right)$ : A divisor $V \subset \mathbb{F}(3,2,1,1)$ from the linear system $|3 M-5 L|$. The 3 -fold $V$ is given by the form

$$
\begin{aligned}
\alpha_{4} x_{1}^{3} & +\alpha_{3} x_{1}^{2} x_{2}+\alpha_{2}^{1} x_{1}^{2} x_{3}+\alpha_{2}^{2} x_{1}^{2} x_{4}+\alpha_{2}^{3} x_{1} x_{2}^{2}+\alpha_{1}^{1} x_{1} x_{2} x_{3}+ \\
& +\alpha_{1}^{2} x_{1} x_{2} x_{4}+\alpha_{0}^{1} x_{1} x_{3}^{2}+\alpha_{0}^{2} x_{1} x_{3} x_{4}+\alpha_{0}^{3} x_{1} x_{4}^{2}+\alpha_{1}^{3} x_{2}^{3}+\alpha_{0}^{4} x_{2}^{2} x_{3}+\alpha_{0}^{5} x_{2}^{2} x_{4} .
\end{aligned}
$$

After a coordinate change similar to one considered in Example 4.12 a (possible) singularity at $O$ is given by the zeroes of the polynomial

$$
x Q_{1}(x, y, z)+Q_{2}(y, z),
$$

where $Q_{1}$ is a polynomial with nonzero constant term and $Q_{2}$ is a polynomial. So $V$ is smooth at a general point of $C$, and by Corollary 2.5 singularities of $V$ are canonical. Note, that $V$ is singular along the curves $x_{1}=x_{2}=\alpha_{0}^{1} x_{3}^{2}+\alpha_{0}^{2} x_{3} x_{4}+$ $\alpha_{0}^{3} x_{4}^{2}=0$.

$\left(T_{20}\right)$ : A divisor $V \subset \mathbb{F}(3,2,2,0)$ from the linear system $|3 M-5 L|$. It's easy to check that $V$ is given by the form

$$
\begin{aligned}
\alpha_{4} x_{1}^{3}+ & \alpha_{3}^{1} x_{1}^{2} x_{2}+\alpha_{3}^{2} x_{1}^{2} x_{3}+\alpha_{1}^{1} x_{1}^{2} x_{4}+\alpha_{2}^{1} x_{1} x_{2}^{2}+\alpha_{2}^{2} x_{1} x_{2} x_{3}+ \\
& +\alpha_{0}^{1} x_{1} x_{2} x_{4}+\alpha_{2}^{3} x_{1} x_{3}^{2}+\alpha_{0}^{2} x_{1} x_{3} x_{4}+\alpha_{1}^{2} x_{2}^{3}+\alpha_{1}^{3} x_{2}^{2} x_{3}+\alpha_{1}^{4} x_{2} x_{3}^{2}+\alpha_{1}^{5} x_{3}^{3} .
\end{aligned}
$$

After a coordinate change similar to one considered in Example 4.12 the singularity at $O$ is given by the zeroes of the polynomial

$$
x^{3}+x^{2} y+x^{2} z+x^{2}+x y^{2}+x y z+x y+x z^{2}+x z+y^{3}+y^{2} z+y z^{2}+z^{3} .
$$

It's easy to check that this singularity is isolated. But here we can not choose "good" weights, so we should resolve the singularity at $O$. After a blow up we obtain two exceptional curves with one point of type $\mathbb{A}_{1}$ on one of them, which means that we have the singularity of type $\mathbb{A}_{3}$. So in the neighbourhood of $O$ the singularity is isomorphic to $\mathbb{A}_{3} \times \mathbb{C}$, and by Corollary 2.5 the singularities of $V$ are canonical. 
$\left(T_{21}\right)$ : A divisor $V \subset \mathbb{F}(3,2,2,1)$ from the linear system $|3 M-6 L|$. It's easy to check that $V$ is given by the form

$$
\begin{aligned}
\alpha_{3} x_{1}^{3}+\alpha_{2}^{1} x_{1}^{2} x_{2}+\alpha_{2}^{2} x_{1}^{2} x_{3}+\alpha_{1}^{1} x_{1}^{2} x_{4}+ & \\
+\alpha_{1}^{2} x_{1} x_{2}^{2}+\alpha_{1}^{3} x_{1} x_{2} x_{3}+\alpha_{0}^{1} x_{1} x_{2} x_{4}+ & \alpha_{1}^{4} x_{1} x_{3}^{2}+\alpha_{0}^{2} x_{1} x_{3} x_{4}+ \\
& +\alpha_{0}^{3} x_{2}^{3}+\alpha_{0}^{4} x_{2}^{2} x_{3}+\alpha_{0}^{5} x_{2} x_{3}^{2}+\alpha_{0}^{6} x_{3}^{3} .
\end{aligned}
$$

After a coordinate change similar to one considered in Example 4.12 the singularity at $O$ is given by the zeroes of the polynomial

$$
x^{3}+x^{2} y+x^{2} z+x^{2}+x y^{2}+x y z+x y+x z^{2}+x z+y^{3}+y^{2} z+y z^{2}+z^{3} .
$$

This polynomial is the same as the corresponding polynomial for the previous variety, so in the neighbourhood of $O$ the singularity is also isomorphic to $\mathbb{A}_{3} \times \mathbb{C}$, and by Corollary 2.5 the singularities of $V$ are canonical.

$\left(T_{22}\right)$ : A divisor $V \subset \mathbb{F}(3,3,1,0)$ from the linear system $|3 M-5 L|$. It's easy to check that $V$ is given by the form

$$
\begin{aligned}
\alpha_{4}^{1} x_{1}^{3}+\alpha_{4}^{2} x_{1}^{2} x_{2}+ & \alpha_{2}^{1} x_{1}^{2} x_{3}+\alpha_{1}^{1} x_{1}^{2} x_{4}+\alpha_{4}^{3} x_{1} x_{2}^{2}+\alpha_{2}^{2} x_{1} x_{2} x_{3}+ \\
& +\alpha_{1}^{2} x_{1} x_{2} x_{4}+\alpha_{0}^{1} x_{1} x_{3}^{2}+\alpha_{4}^{4} x_{2}^{3}+\alpha_{2}^{3} x_{2}^{2} x_{3}+\alpha_{1}^{3} x_{2}^{2} x_{4}+\alpha_{0}^{2} x_{2} x_{3}^{2} .
\end{aligned}
$$

After a coordinate change similar to one considered in Example 4.12 the singularity at $O$ is given by the zeroes of the polynomial

$$
x^{3}+x^{2} y+x^{2} z+x^{2}+x y^{2}+x y z+x y+x z^{2}+y^{3}+y^{2} z+y^{2}+y z^{2} .
$$

If we put $\operatorname{wt}(x)=2, \operatorname{wt}(y)=2$ and $\operatorname{wt}(z)=1$, it's easy to check that the term of the lowest weight defines a singularity that is isolated. The weights coincide with the weights of $\mathbb{A}_{3}$. Then by Theorem 2.10 in the neighbourhood of $O$ the singularity is isomorphic to $\mathbb{A}_{3} \times \mathbb{C}$, and by Corollary 2.5 the singularities of $V$ are canonical.

$\left(T_{23}\right)$ : A divisor $V \subset \mathbb{F}(3,3,2,0)$ from the linear system $|3 M-6 L|$. It's easy to check that $V$ is given by the form

$$
\begin{aligned}
& \alpha_{3}^{1} x_{1}^{3}+\alpha_{3}^{2} x_{1}^{2} x_{2}+\alpha_{2}^{1} x_{1}^{2} x_{3}+ \\
& +\alpha_{0}^{1} x_{1}^{2} x_{4}+\alpha_{3}^{3} x_{1} x_{2}^{2}+\alpha_{2}^{2} x_{1} x_{2} x_{3}+ \\
& \quad \alpha_{0}^{2} x_{1} x_{2} x_{4}+\alpha_{1}^{1} x_{1} x_{3}^{2}+\alpha_{3}^{4} x_{2}^{3}+ \\
& + \\
& +\alpha_{2}^{3} x_{2}^{2} x_{3}+\alpha_{0}^{3} x_{2}^{2} x_{4}+\alpha_{1}^{2} x_{2} x_{3}^{2}+\alpha_{0}^{4} x_{3}^{3} .
\end{aligned}
$$

After a coordinate change similar to one considered in Example 4.12 the singularity at $O$ is given by the zeroes of the polynomial

$$
x^{3}+x^{2} y+x^{2} z+x^{2}+x y^{2}+x y z+x y+x z^{2}+y^{3}+y^{2} z+y^{2}+y z^{2}+z^{3} .
$$

Let $\operatorname{wt}(x)=3, \operatorname{wt}(y)=3$ and $\operatorname{wt}(z)=2$. Then the term of the lowest weight defines an isolated singularity. The weights coincide with the weights of $\mathbb{A}_{2}$. Then by Theorem 2.10 in the neighbourhood of $O$ the singularity is isomorphic to $\mathbb{A}_{2} \times \mathbb{C}$, and by Corollary 2.5 the singularities of $V$ are canonical. 
$\left(T_{24}\right)$ : A divisor $V \subset \mathbb{F}(3,3,2,1)$ from the linear system $|3 M-7 L|$. It's easy to check that $V$ is given by the form

$$
\begin{aligned}
\alpha_{2}^{1} x_{1}^{3}+\alpha_{2}^{2} x_{1}^{2} x_{2}+ & \alpha_{1}^{1} x_{1}^{2} x_{3}+\alpha_{0}^{1} x_{1}^{2} x_{4}+\alpha_{2}^{3} x_{1} x_{2}^{2}+\alpha_{1}^{2} x_{1} x_{2} x_{3}+ \\
& +\alpha_{0}^{2} x_{1} x_{2} x_{4}+\alpha_{0}^{3} x_{1} x_{3}^{2}+\alpha_{2}^{4} x_{2}^{3}+\alpha_{1}^{3} x_{2}^{2} x_{3}+\alpha_{0}^{4} x_{2}^{2} x_{4}+\alpha_{0}^{5} x_{2} x_{3}^{2} .
\end{aligned}
$$

After a coordinate change similar to one considered in Example 4.12 the singularity at $O$ is given by the zeroes of the polynomial

$$
x^{3}+x^{2} y+x^{2} z+x^{2}+x y^{2}+x y z+x y+x z^{2}+y^{3}+y^{2} z+y^{2}+y z^{2} .
$$

This polynomial is the same as the corresponding polynomial for $T_{22}$, so the singularity is isomorphic to $\mathbb{A}_{3} \times \mathbb{C}$ in the neighbourhood of $O$, and by Corollary 2.5] the singularities of $V$ are canonical.

$\left(T_{25}\right)$ : A divisor $V \subset \mathbb{F}(4,1,0,0)$ from the linear system $|3 M-3 L|$. It follows from Proposition 2.19 that the 3 -fold $V$ is given by the form

$$
\begin{aligned}
\alpha_{9} x_{1}^{3}+\alpha_{6} x_{1}^{2} x_{2}+\alpha_{5}^{1} x_{1}^{2} x_{3} & +\alpha_{5}^{2} x_{1}^{2} x_{4}+\alpha_{3} x_{1} x_{2}^{2}+\alpha_{2}^{1} x_{1} x_{2} x_{3}+ \\
& +\alpha_{2}^{2} x_{1} x_{2} x_{4}+\alpha_{1}^{1} x_{1} x_{3}^{2}+\alpha_{1}^{2} x_{1} x_{3} x_{4}+\alpha_{1}^{3} x_{1} x_{4}^{2}+\alpha_{0} x_{2}^{3} .
\end{aligned}
$$

After a coordinate change similar to one considered in Example 4.12 a (possible) singularity at $O$ is given by the zeroes of the polynomial

$$
x Q(x, y, z)+y^{3},
$$

where $Q$ is a polynomial with nonzero constant term. So $V$ is smooth at a general point of $C$, and by Corollary 2.5 singularities of $V$ are canonical. Nevertheless, the 3 -fold $V$ is singular along the possibly reducible curve $x_{1}=x_{2}=\alpha_{1}^{1} x_{3}^{2}+\alpha_{1}^{2} x_{3} x_{4}+$ $\alpha_{1}^{3} x_{4}^{2}=0$.

$\left(T_{26}\right)$ : A divisor $V \subset \mathbb{F}(4,2,0,0)$ from the linear system $|3 M-4 L|$. Then $V$ is given by the form

$$
\begin{aligned}
\alpha_{8} x_{1}^{3} & +\alpha_{6} x_{1}^{2} x_{2}+\alpha_{4}^{1} x_{1}^{2} x_{3}+\alpha_{4}^{2} x_{1}^{2} x_{4}+\alpha_{4}^{3} x_{1} x_{2}^{2}+\alpha_{2}^{1} x_{1} x_{2} x_{3}+ \\
& +\alpha_{2}^{2} x_{1} x_{2} x_{4}+\alpha_{0}^{1} x_{1} x_{3}^{2}+\alpha_{0}^{2} x_{1} x_{3} x_{4}+\alpha_{0}^{3} x_{1} x_{4}^{2}+\alpha_{2}^{3} x_{2}^{3}+\alpha_{0}^{4} x_{2}^{2} x_{3}+\alpha_{0}^{5} x_{2}^{2} x_{4} .
\end{aligned}
$$

After a coordinate change similar to one considered in Example 4.12 a (possible) singularity at $O$ is given by the zeroes of the polynomial

$$
x Q(x, y, z)+y^{2}+y^{3},
$$

where $Q$ is a polynomial with nonzero constant term. So $V$ is smooth at a general point of $C$, and by Corollary 2.5 the singularities of $V$ are canonical. However, the 3 -fold $X$ is singular along the curve $\phi_{\left|-K_{V}\right|}\left(Y_{3}\right)$, where $Y_{3} \subset \mathbb{F}(4,2,0,0)$ is a subscroll $x_{1}=x_{2}=0$. Moreover, $V$ also has non-isolated singularities, i.e. $V$ is singular along the curves give by the equations $x_{1}=x_{2}=\alpha_{0}^{1} x_{3}^{2}+\alpha_{0}^{2} x_{3} x_{4}+\alpha_{0}^{3} x_{4}^{2}=0$.

$\left(T_{27}\right)$ : A divisor $V \subset \mathbb{F}(4,2,1,0)$ from the linear system $|3 M-5 L|$. It's easy to check that $V$ is given by the form

$$
\begin{aligned}
\alpha_{7} x_{1}^{3}+\alpha_{5} x_{1}^{2} x_{2}+\alpha_{4} x_{1}^{2} x_{3} & +\alpha_{3}^{1} x_{1}^{2} x_{4}+\alpha_{3}^{2} x_{1} x_{2}^{2}+\alpha_{2} x_{1} x_{2} x_{3}+ \\
& +\alpha_{1}^{1} x_{1} x_{2} x_{4}+\alpha_{1}^{2} x_{1} x_{3}^{2}+\alpha_{0}^{1} x_{1} x_{3} x_{4}+\alpha_{1}^{3} x_{2}^{3}+\alpha_{0}^{2} x_{2}^{2} x_{3} .
\end{aligned}
$$


After a coordinate change similar to one considered in Example 4.12 the singularity at $O$ is given by the zeroes of the polynomial

$$
x^{3}+x^{2} y+x^{2} z+x^{2}+x y^{2}+x y z+x y+x z^{2}+x z+y^{3}+y^{2} z .
$$

It's easy to check that this singularity is isolated. But here we can not choose "good" weights, so we should resolve the singularity at $O$. After a blow up we obtain two exceptional curves with one point of type $\mathbb{A}_{1}$ on one of them, which means that we have the singularity of type $\mathbb{A}_{3}$. So in the neighbourhood of $O$ the singularity is isomorphic to $\mathbb{A}_{3} \times \mathbb{C}$, and by Corollary 2.5 the singularities of $V$ are canonical.

$\left(T_{28}\right)$ : A divisor $V \subset \mathbb{F}(4,2,1,1)$ from the linear system $|3 M-6 L|$. In this case $V \cong X$. It's easy to check that $V$ is given by the form

$$
\begin{aligned}
\alpha_{6} x_{1}^{3}+\alpha_{4} x_{1}^{2} x_{2} & +\alpha_{3}^{1} x_{1}^{2} x_{3}+\alpha_{3}^{2} x_{1}^{2} x_{4}+\alpha_{2} x_{1} x_{2}^{2}+ \\
& +\alpha_{1}^{1} x_{1} x_{2} x_{3}+\alpha_{1}^{2} x_{1} x_{2} x_{4}+\alpha_{0}^{1} x_{1} x_{3}^{2}+\alpha_{0}^{2} x_{1} x_{3} x_{4}+\alpha_{0}^{3} x_{1} x_{4}^{2}+\alpha_{0}^{4} x_{2}^{3} .
\end{aligned}
$$

After a coordinate change similar to one considered in Example 4.12 a (possible) singularity at $O$ is given by the zeroes of the polynomial

$$
x Q(x, y, z)+y^{3},
$$

where $Q$ is a polynomial with nonzero constant term. So $V$ is smooth at a general point of $C$, and by Corollary 2.5 singularities of $V$ are canonical. However, $V$ always has non-isolated singularities. Namely, the 3 -fold $V$ is singular along the curves $x_{1}=x_{2}=\alpha_{0}^{1} x_{3}^{2}+\alpha_{0}^{2} x_{3} x_{4}+\alpha_{0}^{3} x_{4}^{2}=0$.

$\left(T_{29}\right)$ : A divisor $V \subset \mathbb{F}(4,2,2,0)$ from the linear system $|3 M-6 L|$. It's easy to check that $V$ is given by the form

$$
\begin{aligned}
\alpha_{6} x_{1}^{3}+ & \alpha_{4}^{1} x_{1}^{2} x_{2}+\alpha_{4}^{2} x_{1}^{2} x_{3}+\alpha_{2}^{1} x_{1}^{2} x_{4}+\alpha_{2}^{2} x_{1} x_{2}^{2}+\alpha_{2}^{3} x_{1} x_{2} x_{3}+ \\
& +\alpha_{0}^{1} x_{1} x_{2} x_{4}+\alpha_{2}^{4} x_{1} x_{3}^{2}+\alpha_{0}^{2} x_{1} x_{3} x_{4}+\alpha_{0}^{3} x_{2}^{3}+\alpha_{0}^{4} x_{2}^{2} x_{3}+\alpha_{0}^{5} x_{2} x_{3}^{2}+\alpha_{0}^{6} x_{3}^{3} .
\end{aligned}
$$

After a coordinate change similar to one considered in Example 4.12 the singularity at $O$ is given by the zeroes of the polynomial

$$
x^{3}+x^{2} y+x^{2} z+x^{2}+x y^{2}+x y z+x y+x z^{2}+x z+y^{3}+y^{2} z+y z^{2}+z^{3} .
$$

It's easy to check that this singularity is isolated. But here we can not choose "good" weights, so we should resolve the singularity at $O$. After a blow up we obtain two exceptional curves with one point of type $\mathbb{A}_{1}$ on one of them, which means that we have the singularity of type $\mathbb{A}_{3}$. So in the neighbourhood of $O$ the singularity is isomorphic to $\mathbb{A}_{3} \times \mathbb{C}$, and by Corollary 2.5 the singularities of $V$ are canonical.

$\left(T_{30}\right)$ : A divisor $V \subset \mathbb{F}(4,3,1,0)$ from the linear system $|3 M-6 L|$. It's easy to check that $V$ is given by the form

$$
\begin{aligned}
\alpha_{6} x_{1}^{3}+\alpha_{5} x_{1}^{2} x_{2}+\alpha_{3}^{1} x_{1}^{2} x_{3}+ & \alpha_{2}^{1} x_{1}^{2} x_{4}+\alpha_{4} x_{1} x_{2}^{2}+\alpha_{2}^{2} x_{1} x_{2} x_{3}+ \\
& +\alpha_{1}^{1} x_{1} x_{2} x_{4}+\alpha_{0}^{1} x_{1} x_{3}^{2}+\alpha_{3}^{2} x_{2}^{3}+\alpha_{1}^{2} x_{2}^{2} x_{3}+\alpha_{0}^{2} x_{2}^{2} x_{4} .
\end{aligned}
$$


After a coordinate change similar to one considered in Example 4.12 the singularity at $O$ is given by the zeroes of the polynomial

$$
x^{3}+x^{2} y+x^{2} z+x^{2}+x y^{2}+x y z+x y+x z^{2}+y^{3}+y^{2} z+y^{2} .
$$

If we put $\operatorname{wt}(x)=2, \operatorname{wt}(y)=2$ and $\operatorname{wt}(z)=1$, it is not hard to see that the term of the lowest weight defines an isolated singularity. The weights coincide with the weights of $\mathbb{A}_{3}$. Then by Theorem 2.10 in the neighbourhood of $O$ the singularity is isomorphic to $\mathbb{A}_{3} \times \mathbb{C}$, and by Corollary 2.5 the singularities of $V$ are canonical.

$\left(T_{31}\right)$ : A divisor $V \subset \mathbb{F}(4,3,2,0)$ from the linear system $|3 M-7 L|$. It's easy to check that $V$ is given by the form

$$
\begin{aligned}
\alpha_{5} x_{1}^{3}+\alpha_{4} x_{1}^{2} x_{2}+\alpha_{3}^{1} x_{1}^{2} x_{3}+ & \alpha_{1}^{1} x_{1}^{2} x_{4}+\alpha_{3}^{2} x_{1} x_{2}^{2}+\alpha_{2}^{1} x_{1} x_{2} x_{3}+ \\
& +\alpha_{0}^{1} x_{1} x_{2} x_{4}+\alpha_{1}^{2} x_{1} x_{3}^{2}+\alpha_{2}^{2} x_{2}^{3}+\alpha_{1}^{3} x_{2}^{2} x_{3}+\alpha_{0}^{2} x_{2} x_{3}^{2} .
\end{aligned}
$$

After a coordinate change similar to one considered in Example 4.12 the singularity at $O$ is given by the zeroes of the polynomial

$$
x^{3}+x^{2} y+x^{2} z+x^{2}+x y^{2}+x y z+x y+x z^{2}+y^{3}+y^{2} z+y z^{2} .
$$

If we put $\operatorname{wt}(x)=2, \operatorname{wt}(y)=2$ and $\operatorname{wt}(z)=1$, it is easy to check that the term of the lowest weight defines a singularity that is isolated. The weights coincide with the weights of $\mathbb{A}_{3}$. Then by Theorem 2.10 in the neighbourhood of $O$ the singularity is isomorphic to $\mathbb{A}_{3} \times \mathbb{C}$, and by Corollary 2.5 the singularities of $V$ are canonical.

$\left(T_{32}\right)$ : A divisor $V \subset \mathbb{F}(4,3,2,1)$ from the linear system $|3 M-8 L|$. It's easy to check that $V$ is given by the form

$$
\begin{aligned}
\alpha_{4} x_{1}^{3}+\alpha_{3} x_{1}^{2} x_{2}+\alpha_{2}^{1} x_{1}^{2} x_{3} & +\alpha_{1}^{1} x_{1}^{2} x_{4}+\alpha_{2}^{2} x_{1} x_{2}^{2}+ \\
& +\alpha_{1}^{2} x_{1} x_{2} x_{3}+\alpha_{0}^{1} x_{1} x_{2} x_{4}+\alpha_{0}^{2} x_{1} x_{3}^{2}+\alpha_{1}^{3} x_{2}^{3}+\alpha_{0}^{3} x_{2}^{2} x_{3} .
\end{aligned}
$$

After a coordinate change similar to one considered in Example 4.12 the singularity at $O$ is given by the zeroes of the polynomial

$$
x^{3}+x^{2} y+x^{2} z+x^{2}+x y^{2}+x y z+x y+x z^{2}+y^{3}+y^{2} z .
$$

It's easy to check that this singularity is isolated. But here we can not choose "good" weights, so we should resolve the singularity at $O$. After two blow ups we obtain a smooth surface and exceptional curves will form a Dynkin diagram $\mathbb{A}_{4}$. So in the neighbourhood of $O$ the singularity is isomorphic to $\mathbb{A}_{4} \times \mathbb{C}$, and by Corollary 2.5 the singularities of $V$ are canonical.

$\left(T_{33}\right)$ : A divisor $V \subset \mathbb{F}(4,3,3,0)$ from the linear system $|3 M-8 L|$. It's easy to check that $V$ is given by the form

$$
\begin{aligned}
\alpha_{4} x_{1}^{3}+\alpha_{3}^{1} x_{1}^{2} x_{2}+\alpha_{3}^{2} x_{1}^{2} x_{3}+\alpha_{0} x_{1}^{2} x_{4}+\alpha_{2}^{1} x_{1} x_{2}^{2}+\alpha_{2}^{2} x_{1} x_{2} x_{3}+ \\
+\alpha_{2}^{3} x_{1} x_{3}^{2}+\alpha_{1}^{1} x_{2}^{3}+\alpha_{1}^{2} x_{2}^{2} x_{3}+\alpha_{1}^{3} x_{2} x_{3}^{2}+\alpha_{1}^{4} x_{3}^{3} .
\end{aligned}
$$

After a coordinate change similar to one considered in Example 4.12 the singularity at $O$ is given by the zeroes of the polynomial

$$
x^{3}+x^{2} y+x^{2} z+x^{2}+x y^{2}+x y z+x z^{2}+y^{3}+y^{2} z+y z^{2}+z^{3} .
$$


Put $\operatorname{wt}(x)=3, \operatorname{wt}(y)=2$ and $\operatorname{wt}(z)=2$. It is easy to check that the term of the lowest weight defines an isolated singularity. The weights coincide with the weights of $\mathbb{D}_{4}$. Then by Theorem 2.10 in the neighbourhood of $O$ the singularity is isomorphic to $\mathbb{D}_{4} \times \mathbb{C}$, and by Corollary 2.5 the singularities of $V$ are canonical.

$\left(T_{34}\right)$ : A divisor $V \subset \mathbb{F}(4,3,3,1)$ from the linear system $|3 M-9 L|$. It's easy to check that $V$ is given by the form

$$
\begin{aligned}
\alpha_{3} x_{1}^{3}+\alpha_{2}^{1} x_{1}^{2} x_{2}+\alpha_{2}^{2} x_{1}^{2} x_{3}+\alpha_{0}^{1} x_{1}^{2} x_{4}+\alpha_{1}^{1} x_{1} x_{2}^{2}+\alpha_{1}^{2} x_{1} x_{2} x_{3}+ & \\
& +\alpha_{1}^{3} x_{1} x_{3}^{2}+\alpha_{0}^{2} x_{2}^{3}+\alpha_{0}^{3} x_{2}^{2} x_{3}+\alpha_{0}^{4} x_{2} x_{3}^{2}+\alpha_{0}^{5} x_{3}^{3} .
\end{aligned}
$$

After a coordinate change similar to one considered in Example 4.12 the singularity at $O$ is given by the zeroes of the polynomial

$$
x^{3}+x^{2} y+x^{2} z+x^{2}+x y^{2}+x y z+x z^{2}+y^{3}+y^{2} z+y z^{2}+z^{3} .
$$

This polynomial is the same as the corresponding polynomial for $T_{33}$, so the singularity is isomorphic to $\mathbb{D}_{4} \times \mathbb{C}$ in the neighbourhood of $O$, and by Corollary 2.5] the singularities of $V$ are canonical.

$\left(T_{35}\right)$ : A divisor $V \subset \mathbb{F}(4,4,2,0)$ from the linear system $|3 M-8 L|$. It's easy to check that $V$ is given by the form

$$
\begin{aligned}
\alpha_{4}^{1} x_{1}^{3}+\alpha_{4}^{2} x_{1}^{2} x_{2}+ & \alpha_{2}^{1} x_{1}^{2} x_{3}+\alpha_{0}^{1} x_{1}^{2} x_{4}+\alpha_{4}^{3} x_{1} x_{2}^{2}+\alpha_{2}^{2} x_{1} x_{2} x_{3}+ \\
& +\alpha_{0}^{2} x_{1} x_{2} x_{4}+\alpha_{0}^{3} x_{1} x_{3}^{2}+\alpha_{4}^{4} x_{2}^{3}+\alpha_{2}^{3} x_{2}^{2} x_{3}+\alpha_{0}^{4} x_{2}^{2} x_{4}+\alpha_{0}^{5} x_{2} x_{3}^{2} .
\end{aligned}
$$

After a coordinate change similar to one considered in Example 4.12 the singularity at $O$ is given by the zeroes of the polynomial

$$
x^{3}+x^{2} y+x^{2} z+x^{2}+x y^{2}+x y z+x y+x z^{2}+y^{3}+y^{2} z+y^{2}+y z^{2} .
$$

This polynomial is the same as the corresponding polynomial for $T_{22}$, so the singularity is isomorphic to $\mathbb{A}_{3} \times \mathbb{C}$ in the neighbourhood of $O$, and by Corollary 2.5 the singularities of $V$ are canonical.

$\left(T_{36}\right)$ : A divisor $V \subset \mathbb{F}(5,2,0,0)$ from the linear system $|3 M-5 L|$. It's easy to check that $V$ is given by the form

$$
\begin{aligned}
\alpha_{10} x_{1}^{3}+\alpha_{7} x_{1}^{2} x_{2}+\alpha_{5}^{1} x_{1}^{2} x_{3} & +\alpha_{5}^{2} x_{1}^{2} x_{4}+\alpha_{4} x_{1} x_{2}^{2}+\alpha_{2}^{1} x_{1} x_{2} x_{3}+ \\
& +\alpha_{2}^{2} x_{1} x_{2} x_{4}+\alpha_{0}^{1} x_{1} x_{3}^{2}+\alpha_{0}^{2} x_{1} x_{3} x_{4}+\alpha_{0}^{3} x_{1} x_{4}^{2}+\alpha_{1} x_{2}^{3} .
\end{aligned}
$$

After a coordinate change similar to one considered in Example 4.12 a (possible) singularity at $O$ is given by he zeroes of the polynomial

$$
x Q(x, y, z)+y^{3},
$$

where $Q$ is a polynomial with nonzero constant term. So $V$ is smooth at a general point of $C$, and by Corollary 2.5 the singularities of $V$ are canonical. On the other hand, the 3 -fold $V$ is singular along the curves $x_{1}=x_{2}=\alpha_{0}^{1} x_{3}^{2}+\alpha_{0}^{2} x_{3} x_{4}+\alpha_{0}^{3} x_{4}^{2}=0$.

$\left(T_{37}\right)$ : A divisor $V \subset \mathbb{F}(5,2,1,0)$ from the linear system $|3 M-6 L|$. It's easy to check that $V$ is given by the form

$$
\begin{aligned}
\alpha_{9} x_{1}^{3}+\alpha_{6} x_{1}^{2} x_{2}+\alpha_{5} x_{1}^{2} x_{3} & +\alpha_{4} x_{1}^{2} x_{4}+\alpha_{3} x_{1} x_{2}^{2}+ \\
+ & \alpha_{2} x_{1} x_{2} x_{3}+\alpha_{1}^{1} x_{1} x_{2} x_{4}+\alpha_{1}^{2} x_{1} x_{3}^{2}+\alpha_{0}^{1} x_{1} x_{3} x_{4}+\alpha_{0}^{2} x_{2}^{3} .
\end{aligned}
$$


After a coordinate change similar to one considered in Example 4.12 a (possible) singularity at $O$ is given by the zeroes of the polynomial

$$
x^{3}+x^{2} y+x^{2} z+x^{2}+x y^{2}+x y z+x y+x z^{2}+x z+y^{3} .
$$

After a coordinate change $x^{\prime}=x, y^{\prime}=y, z^{\prime}=x+y+z$ one can see that this singularity is Du Val of type $\mathbb{A}_{2}$. So the singularity on $V$ in the neighbourhood of the point $O$ is isomorphic to $\mathbb{A}_{2} \times \mathbb{C}$, and by Corollary 2.5 the singularities of $V$ are canonical.

$\left(T_{38}\right)$ : A divisor $V \subset \mathbb{F}(5,3,1,0)$ from the linear system $|3 M-7 L|$. It's easy to check that $V$ is given by the form

$$
\begin{aligned}
\alpha_{8} x_{1}^{3}+\alpha_{6} x_{1}^{2} x_{2}+\alpha_{4}^{1} x_{1}^{2} x_{3} & +\alpha_{3} x_{1}^{2} x_{4}+\alpha_{4}^{2} x_{1} x_{2}^{2}+ \\
& +\alpha_{2}^{1} x_{1} x_{2} x_{3}+\alpha_{1} x_{1} x_{2} x_{4}+\alpha_{0}^{1} x_{1} x_{3}^{2}+\alpha_{2}^{2} x_{2}^{3}+\alpha_{0}^{2} x_{2}^{2} x_{3} .
\end{aligned}
$$

After a coordinate change similar to one considered in Example 4.12 the singularity at the point $O$ is given by the zeroes of the polynomial

$$
x^{3}+x^{2} y+x^{2} z+x^{2}+x y^{2}+x y z+x y+x z^{2}+y^{3}+y^{2} z .
$$

This polynomial is the same as the corresponding polynomial for $T_{32}$, so the singularity is isomorphic to $\mathbb{A}_{4} \times \mathbb{C}$ in the neighbourhood of the point $O$, and by Corollary 2.5 the singularities of $V$ are canonical.

$\left(T_{39}\right)$ : A divisor $V \subset \mathbb{F}(5,3,2,0)$ from the linear system $|3 M-8 L|$. It's easy to check that $V$ is given by the form

$$
\begin{aligned}
\alpha_{7} x_{1}^{3}+\alpha_{5} x_{1}^{2} x_{2}+\alpha_{4} x_{1}^{2} x_{3} & +\alpha_{2}^{1} x_{1}^{2} x_{4}+\alpha_{3} x_{1} x_{2}^{2}+ \\
& +\alpha_{2}^{2} x_{1} x_{2} x_{3}+\alpha_{0}^{1} x_{1} x_{2} x_{4}+\alpha_{1}^{1} x_{1} x_{3}^{2}+\alpha_{1}^{2} x_{2}^{3}+\alpha_{0}^{2} x_{2}^{2} x_{3} .
\end{aligned}
$$

After a coordinate change similar to one considered in Example 4.12 the singularity at $O$ is given by the zeroes of the polynomial

$$
x^{3}+x^{2} y+x^{2} z+x^{2}+x y^{2}+x y z+x y+x z^{2}+y^{3}+y^{2} z .
$$

This polynomial is the same as the corresponding polynomial for $T_{32}$, so the singularity is isomorphic to $\mathbb{A}_{5} \times \mathbb{C}$ in the neighbourhood of $O$, and by Corollary 2.5 the singularities of $V$ are canonical.

$\left(T_{40}\right)$ : A divisor $V \subset \mathbb{F}(5,3,2,1)$ from the linear system $|3 M-9 L|$. It's easy to check that $V$ is given by the form

$$
\begin{aligned}
\alpha_{6} x_{1}^{3}+\alpha_{4} x_{1}^{2} x_{2}+\alpha_{3} x_{1}^{2} x_{3}+\alpha_{2}^{1} x_{1}^{2} x_{4} & +\alpha_{2}^{2} x_{1} x_{2}^{2}+ \\
& +\alpha_{1} x_{1} x_{2} x_{3}+\alpha_{0}^{1} x_{1} x_{2} x_{4}+\alpha_{0}^{2} x_{1} x_{3}^{2}+\alpha_{0}^{3} x_{2}^{3} .
\end{aligned}
$$

After a coordinate change similar to one considered in Example 4.12 the singularity at $O$ is given by the zeroes of the polynomial

$$
x^{3}+x^{2} y+x^{2} z+x^{2}+x y^{2}+x y z+x y+x z^{2}+y^{3} .
$$

It's easy to check that this singularity is isolated. But here we can not choose "good" weights, so we should resolve the singularity at $O$. After three blow ups we obtain a smooth surface and exceptional curves will form a Dynkin diagram $\mathbb{A}_{5}$. So in the neighbourhood of $O$ the singularity is isomorphic to $\mathbb{A}_{5} \times \mathbb{C}$, and by Corollary 2.5 the singularities of $V$ are canonical. 
$\left(T_{41}\right):$ A divisor $V \subset \mathbb{F}(5,3,3,0)$ from the linear system $|3 M-9 L|$. It's easy to check that $V$ is given by the form

$$
\begin{aligned}
\alpha_{6} x_{1}^{3}+\alpha_{4}^{1} x_{1}^{2} x_{2}+\alpha_{4}^{2} x_{1}^{2} x_{3}+\alpha_{1} x_{1}^{2} x_{4}+\alpha_{2}^{1} x_{1} x_{2}^{2}+\alpha_{2}^{2} x_{1} x_{2} x_{3}+ \\
+\alpha_{2}^{3} x_{1} x_{3}^{2}+\alpha_{0}^{1} x_{2}^{3}+\alpha_{0}^{2} x_{2}^{2} x_{3}+\alpha_{0}^{3} x_{2} x_{3}^{2}+\alpha_{0}^{4} x_{3}^{3} .
\end{aligned}
$$

After a coordinate change similar to one considered in Example 4.12 the singularity at $O$ is given by the zeroes of the polynomial

$$
x^{3}+x^{2} y+x^{2} z+x^{2}+x y^{2}+x y z+x z^{2}+y^{3}+y^{2} z+y z^{2}+z^{3} .
$$

This polynomial is the same as the corresponding polynomial for $T_{33}$, so the singularity in the neighbourhood of the point $O$ is isomorphic to $\mathbb{D}_{4} \times \mathbb{C}$, and by Corollary 2.5 the singularities of $V$ are canonical.

$\left(T_{42}\right)$ : A divisor $V \subset \mathbb{F}(5,4,2,0)$ from the linear system $|3 M-9 L|$. It's easy to check that $V$ is given by the form

$$
\begin{aligned}
\alpha_{6} x_{1}^{3}+\alpha_{5} x_{1}^{2} x_{2}+\alpha_{3}^{1} x_{1}^{2} x_{3} & +\alpha_{1}^{1} x_{1}^{2} x_{4}+\alpha_{4} x_{1} x_{2}^{2}+ \\
& +\alpha_{2} x_{1} x_{2} x_{3}+\alpha_{0}^{1} x_{1} x_{2} x_{4}+\alpha_{0}^{2} x_{1} x_{3}^{2}+\alpha_{3}^{2} x_{2}^{3}+\alpha_{1}^{2} x_{2}^{2} x_{3} .
\end{aligned}
$$

After a coordinate change similar to one considered in Example 4.12 the singularity at $O$ is given by the zeroes of the polynomial

$$
x^{3}+x^{2} y+x^{2} z+x^{2}+x y^{2}+x y z+x y+x z^{2}+y^{3}+y^{2} z .
$$

This polynomial is the same as the corresponding polynomial for $T_{32}$, so the singularity is isomorphic to $\mathbb{A}_{4} \times \mathbb{C}$ in the neighbourhood of $O$, and by Corollary 2.5 the singularities of $V$ are canonical.

$\left(T_{43}\right):$ A divisor $V \subset \mathbb{F}(5,4,3,0)$ from the linear system $|3 M-10 L|$. It's easy to check that $V$ is given by the form

$$
\begin{aligned}
\alpha_{5} x_{1}^{3}+\alpha_{4} x_{1}^{2} x_{2}+\alpha_{3}^{1} x_{1}^{2} x_{3}+\alpha_{0}^{1} x_{1}^{2} x_{4}+\alpha_{3}^{2} x_{1} x_{2}^{2}+\alpha_{2}^{1} x_{1} x_{2} x_{3}+ \\
+\alpha_{1}^{1} x_{1} x_{3}^{2}+\alpha_{2}^{2} x_{2}^{3}+\alpha_{1}^{2} x_{2}^{2} x_{3}+\alpha_{0}^{2} x_{2} x_{3}^{2} .
\end{aligned}
$$

After a coordinate change similar to one considered in Example 4.12 the singularity at $O$ is given by the zeroes of the polynomial

$$
x^{3}+x^{2} y+x^{2} z+x^{2}+x y^{2}+x y z+x z^{2}+y^{3}+y^{2} z+y z^{2} .
$$

If we put $\operatorname{wt}(x)=3, \operatorname{wt}(y)=2$ and $\operatorname{wt}(z)=2$, it's easy to check that the term of the lowest weight defines a singularity that is isolated. The weights coincide with the weights of $\mathbb{D}_{4}$. Then by Theorem 2.10 in the neighbourhood of $O$ the singularity is isomorphic to $\mathbb{D}_{4} \times \mathbb{C}$, and by Corollary 2.5 the singularities of $V$ are canonical.

$\left(T_{44}\right)$ : A divisor $V \subset \mathbb{F}(5,4,3,1)$ from the linear system $|3 M-11 L|$. It's easy to check that $V$ is given by the form

$$
\begin{aligned}
\alpha_{4} x_{1}^{3}+\alpha_{3} x_{1}^{2} x_{2}+\alpha_{2}^{1} x_{1}^{2} x_{3}+\alpha_{0}^{1} x_{1}^{2} x_{4}+ & \alpha_{2}^{2} x_{1} x_{2}^{2}+ \\
& +\alpha_{1}^{1} x_{1} x_{2} x_{3}+\alpha_{0}^{2} x_{1} x_{3}^{2}+\alpha_{1}^{2} x_{2}^{3}+\alpha_{0}^{3} x_{2}^{2} x_{3} .
\end{aligned}
$$

After a coordinate change similar to one considered in Example 4.12 the singularity at $O$ is given by the zeroes of the polynomial

$$
x^{3}+x^{2} y+x^{2} z+x^{2}+x y^{2}+x y z+x z^{2}+y^{3}+y^{2} z
$$


If we put $\operatorname{wt}(x)=4, \operatorname{wt}(y)=3$ and $\operatorname{wt}(z)=2$, it's easy to check that the term of the lowest weight defines a singularity that is isolated. The weights coincide with the weights of $\mathbb{D}_{5}$. Then by Theorem 2.10 in the neighbourhood of $O$ the singularity is isomorphic to $\mathbb{D}_{5} \times \mathbb{C}$, and by Corollary 2.5 the singularities of $V$ are canonical.

$\left(T_{45}\right)$ : A divisor $V \subset \mathbb{F}(6,2,0,0)$ from the linear system $|3 M-6 L|$. In this case $\phi_{\left|-K_{V}\right|}$ contracts a surface $x_{1}=x_{2}=0$ to a curve which is singular on $X$. It's easy to check that $V$ is given by the form

$$
\begin{aligned}
\alpha_{12} x_{1}^{3}+\alpha_{8} x_{1}^{2} x_{2}+\alpha_{6}^{1} x_{1}^{2} x_{3} & +\alpha_{6}^{2} x_{1}^{2} x_{4}+\alpha_{4} x_{1} x_{2}^{2}+\alpha_{2}^{1} x_{1} x_{2} x_{3}+ \\
& +\alpha_{2}^{2} x_{1} x_{2} x_{4}+\alpha_{0}^{1} x_{1} x_{3}^{2}+\alpha_{0}^{2} x_{1} x_{3} x_{4}+\alpha_{0}^{3} x_{1} x_{4}^{2}+\alpha_{0}^{4} x_{2}^{3} .
\end{aligned}
$$

After a coordinate change similar to one considered in Example 4.12 a (possible) singularity at $O$ is given by the zeroes of the polynomial

$$
x Q(x, y, z)+y^{3},
$$

where $Q$ is a polynomial with nonzero constant term. So $V$ is smooth at a general point of $C$, and by Corollary 2.5 the singularities of $V$ are canonical. However, the 3 -fold $V$ is singular along the curves $x_{1}=x_{2}=\alpha_{0}^{1} x_{3}^{2}+\alpha_{0}^{2} x_{3} x_{4}+\alpha_{0}^{3} x_{4}^{2}=0$.

$\left(T_{46}\right)$ : A divisor $V \subset \mathbb{F}(6,3,1,0)$ from the linear system $|3 M-8 L|$. It's easy to check that $V$ is given by the form

$$
\begin{aligned}
\alpha_{10} x_{1}^{3}+\alpha_{7} x_{1}^{2} x_{2}+\alpha_{5} x_{1}^{2} x_{3}+\alpha_{4}^{1} x_{1}^{2} x_{4} & +\alpha_{4}^{2} x_{1} x_{2}^{2}+ \\
& +\alpha_{2} x_{1} x_{2} x_{3}+\alpha_{1}^{1} x_{1} x_{2} x_{4}+\alpha_{0} x_{1} x_{3}^{2}+\alpha_{1}^{2} x_{2}^{3} .
\end{aligned}
$$

After a coordinate change similar to one considered in Example 4.12 the singularity at $O$ is given by the zeroes of the polynomial

$$
x^{3}+x^{2} y+x^{2} z+x^{2}+x y^{2}+x y z+x y+x z^{2}+y^{3} .
$$

This polynomial is the same as the corresponding polynomial for $T_{40}$, so the singularity is isomorphic to $\mathbb{A}_{5} \times \mathbb{C}$ in the neighbourhood of $O$, and by Corollary 2.5] the singularities of $V$ are canonical.

$\left(T_{47}\right)$ : A divisor $V \subset \mathbb{F}(6,3,2,0)$ from the linear system $|3 M-9 L|$. It's easy to check that $V$ is given by the form

$$
\begin{aligned}
\alpha_{9} x_{1}^{3}+\alpha_{6} x_{1}^{2} x_{2}+\alpha_{5} x_{1}^{2} x_{3}+\alpha_{3}^{1} x_{1}^{2} x_{4} & +\alpha_{3}^{2} x_{1} x_{2}^{2}+ \\
& +\alpha_{2} x_{1} x_{2} x_{3}+\alpha_{0}^{1} x_{1} x_{2} x_{4}+\alpha_{1} x_{1} x_{3}^{2}+\alpha_{0}^{2} x_{2}^{3} .
\end{aligned}
$$

After a coordinate change similar to one considered in Example 4.12 the singularity at $O$ is given by the zeroes of the polynomial

$$
x^{3}+x^{2} y+x^{2} z+x^{2}+x y^{2}+x y z+x y+x z^{2}+y^{3} .
$$

This polynomial is the same as the corresponding polynomial for $T_{40}$, so the singularity is isomorphic to $\mathbb{A}_{5} \times \mathbb{C}$ in the neighbourhood of the point $O$, and by Corollary 2.5 the singularities of $V$ are canonical. 
$\left(T_{48}\right)$ : A divisor $V \subset \mathbb{F}(6,4,2,0)$ from the linear system $|3 M-10 L|$. It's easy to check that $V$ is given by the form

$$
\begin{aligned}
\alpha_{8} x_{1}^{3}+\alpha_{6} x_{1}^{2} x_{2}+\alpha_{4}^{1} x_{1}^{2} x_{3}+\alpha_{2}^{1} x_{1}^{2} x_{4}+ & \alpha_{4}^{2} x_{1} x_{2}^{2}+\alpha_{2}^{2} x_{1} x_{2} x_{3}+ \\
& +\alpha_{0}^{1} x_{1} x_{2} x_{4}+\alpha_{0}^{2} x_{1} x_{3}^{2}+\alpha_{2}^{3} x_{2}^{3}+\alpha_{0}^{3} x_{2}^{2} x_{3} .
\end{aligned}
$$

After a coordinate change similar to one considered in Example 4.12 the singularity at $O$ is given by the zeroes of the polynomial

$$
x^{3}+x^{2} y+x^{2} z+x^{2}+x y^{2}+x y z+x y+x z^{2}+y^{3}+y^{2} z .
$$

This polynomial is the same as the corresponding polynomial for $T_{32}$, so in the neighbourhood of the point $O$ the singularity is isomorphic to $\mathbb{A}_{4} \times \mathbb{C}$, and by Corollary 2.5 the singularities of $V$ are canonical.

$\left(T_{49}\right)$ : A divisor $V \subset \mathbb{F}(6,4,3,0)$ from the linear system $|3 M-11 L|$. It's easy to check that $V$ is given by the form

$$
\begin{aligned}
\alpha_{7} x_{1}^{3}+\alpha_{5} x_{1}^{2} x_{2}+\alpha_{4} x_{1}^{2} x_{3}+\alpha_{1}^{1} x_{1}^{2} x_{4}+ & \alpha_{3} x_{1} x_{2}^{2}+ \\
& +\alpha_{2} x_{1} x_{2} x_{3}+\alpha_{1}^{2} x_{1} x_{3}^{2}+\alpha_{1}^{3} x_{2}^{3}+\alpha_{0} x_{2}^{2} x_{3} .
\end{aligned}
$$

After a coordinate change similar to one considered in Example 4.12 the singularity at $O$ is given by the zeroes of the polynomial

$$
x^{3}+x^{2} y+x^{2} z+x^{2}+x y^{2}+x y z+x z^{2}+y^{3}+y^{2} z .
$$

This polynomial is the same as the corresponding polynomial for $T_{44}$, so the singularity is isomorphic to $\mathbb{D}_{5} \times \mathbb{C}$ in the neighbourhood of the point $O$, and by Corollary 2.5 the singularities of $V$ are canonical.

$\left(T_{50}\right):$ A divisor $V \subset \mathbb{F}(6,4,3,1)$ from the linear system $|3 M-12 L|$. It's easy to check that $V$ is given by the form

$$
\alpha_{6} x_{1}^{3}+\alpha_{4} x_{1}^{2} x_{2}+\alpha_{3} x_{1}^{2} x_{3}+\alpha_{1}^{1} x_{1}^{2} x_{4}+\alpha_{2} x_{1} x_{2}^{2}+\alpha_{1}^{2} x_{1} x_{2} x_{3}+\alpha_{0}^{1} x_{1} x_{3}^{2}+\alpha_{0}^{2} x_{2}^{3} .
$$

After a coordinate change similar to one considered in Example 4.12 the singularity at $O$ is given by the zeroes of the polynomial

$$
x^{3}+x^{2} y+x^{2} z+x^{2}+x y^{2}+x y z+x z^{2}+y^{3} .
$$

Let us put $\operatorname{wt}(x)=6, \operatorname{wt}(y)=4$ and $\operatorname{wt}(z)=3$. Then the term of the lowest weight defines a singularity that is isolated. Moreover, the weights coincide with the weights of $\mathbb{E}_{6}$. Then by Theorem 2.10 in the neighbourhood of $O$ the singularity is isomorphic to $\mathbb{E}_{6} \times \mathbb{C}$, and by Corollary 2.5 the singularities of $V$ are canonical.

$\left(T_{51}\right)$ : A divisor $V \subset \mathbb{F}(6,4,4,0)$ from the linear system $|3 M-12 L|$. It's easy to check that $V$ is given by the form

$$
\begin{aligned}
\alpha_{6} x_{1}^{3}+\alpha_{4}^{1} x_{1}^{2} x_{2}+ & \alpha_{4}^{2} x_{1}^{2} x_{3}+\alpha_{0}^{1} x_{1}^{2} x_{4}+ \\
& +\alpha_{2}^{1} x_{1} x_{2}^{2}+\alpha_{2}^{2} x_{1} x_{2} x_{3}+ \\
& \alpha_{2}^{3} x_{1} x_{3}^{2}+ \\
& +\alpha_{0}^{1} x_{2}^{3}+\alpha_{0}^{2} x_{2}^{2} x_{3}+\alpha_{0}^{3} x_{2} x_{3}^{2}+\alpha_{0}^{4} x_{3}^{3} .
\end{aligned}
$$

After a coordinate change similar to one considered in Example 4.12 the singularity at $O$ is given by the zeroes of the polynomial

$$
x^{2}+P_{3}(y, z)+x P_{2}(y, z),
$$


where $P_{i}$ is a homogeneous polynomial of degree $i$. Let $\operatorname{wt}(x)=3$, $\operatorname{wt}(y)=2$ and $\operatorname{wt}(z)=2$. Then the term of the lowest weight defines an isolated singularity. Moreover, the considered weights coincide with the weights of Du Val singularity $\mathbb{D}_{4}$. Then by Theorem 2.10 in the neighbourhood of $O$ the singularity is isomorphic to $\mathbb{D}_{4} \times \mathbb{C}$, and by Corollary 2.5 the singularities of $V$ are canonical.

$\left(T_{52}\right):$ A divisor $V \subset \mathbb{F}(6,5,3,0)$ from the linear system $|3 M-12 L|$. It's easy to check that $V$ is given by the form

$$
\begin{aligned}
\alpha_{6} x_{1}^{3}+\alpha_{5} x_{1}^{2} x_{2}+\alpha_{3}^{1} x_{1}^{2} x_{3}+ & \alpha_{0}^{1} x_{1}^{2} x_{4}+ \\
& +\alpha_{4} x_{1} x_{2}^{2}+\alpha_{2} x_{1} x_{2} x_{3}+\alpha_{0}^{2} x_{1} x_{3}^{2}+\alpha_{3}^{2} x_{2}^{3}+\alpha_{1} x_{2}^{2} x_{3} .
\end{aligned}
$$

After a coordinate change similar to one considered in Example 4.12 the singularity at $O$ is given by the zeroes of the polynomial

$$
x^{3}+x^{2} y+x^{2} z+x^{2}+x y^{2}+x y z+x z^{2}+y^{3}+y^{2} z .
$$

If we put $\operatorname{wt}(x)=4, \operatorname{wt}(y)=3$ and $\operatorname{wt}(z)=2$, it is easy to check that the term of the lowest weight defines an isolated singularity. Moreover, the weights coincide with the weights of $\mathbb{D}_{5}$. Then by Theorem 2.10 in the neighbourhood of $O$ the singularity is isomorphic to $\mathbb{D}_{5} \times \mathbb{C}$, and by Corollary 2.5 the singularities of $V$ are canonical.

$\left(T_{53}\right)$ : A divisor $V \subset \mathbb{F}(7,3,1,0)$ from the linear system $|3 M-9 L|$. It's easy to check that $V$ is given by the form

$$
\begin{aligned}
\alpha_{12} x_{1}^{3}+\alpha_{8} x_{1}^{2} x_{2}+\alpha_{6} x_{1}^{2} x_{3}+\alpha_{5} x_{1}^{2} x_{4} & +\alpha_{4} x_{1} x_{2}^{2}+ \\
& +\alpha_{2} x_{1} x_{2} x_{3}+\alpha_{1} x_{1} x_{2} x_{4}+\alpha_{0}^{1} x_{1} x_{3}^{2}+\alpha_{0}^{2} x_{2}^{3} .
\end{aligned}
$$

After a coordinate change similar to one considered in Example 4.12 the singularity at $O$ is given by the zeroes of the polynomial

$$
x^{3}+x^{2} y+x^{2} z+x^{2}+x y^{2}+x y z+x y+x z^{2}+y^{3} .
$$

It's easy to check that this singularity is isolated. But here we can not choose "good" weights, so we should resolve the singularity at $O$. After three blow ups we obtain a smooth surface and exceptional curves will form a Dynkin diagram $\mathbb{A}_{5}$. So in the neighbourhood of $O$ the singularity is isomorphic to $\mathbb{A}_{5} \times \mathbb{C}$, and by Corollary 2.5 the singularities of $V$ are canonical.

$\left(T_{54}\right)$ : A divisor $V \subset \mathbb{F}(7,4,2,0)$ from the linear system $|3 M-11 L|$. It's easy to check that $V$ is given by the form

$$
\begin{aligned}
\alpha_{10} x_{1}^{3}+\alpha_{7} x_{1}^{2} x_{2}+\alpha_{5} x_{1}^{2} x_{3} & +\alpha_{3} x_{1}^{2} x_{4}+ \\
& +\alpha_{4} x_{1} x_{2}^{2}+\alpha_{2} x_{1} x_{2} x_{3}+\alpha_{0}^{1} x_{1} x_{2} x_{4}+\alpha_{0}^{2} x_{1} x_{3}^{2}+\alpha_{1} x_{2}^{3} .
\end{aligned}
$$

After a coordinate change similar to one considered in Example 4.12 the singularity at $O$ is given by the zeroes of the polynomial

$$
x^{3}+x^{2} y+x^{2} z+x^{2}+x y^{2}+x y z+x y+x z^{2}+y^{3} .
$$

It's easy to check that this singularity is isolated. But here we can not choose "good" weights, so we should resolve the singularity at $O$. After three blow ups we obtain a smooth surface and exceptional curves will form a Dynkin diagram 
$\mathbb{A}_{5}$. So in the neighbourhood of $O$ the singularity is isomorphic to $\mathbb{A}_{5} \times \mathbb{C}$, and by Corollary 2.5 the singularities of $V$ are canonical.

$\left(T_{55}\right)$ : A divisor $V \subset \mathbb{F}(7,4,3,0)$ from the linear system $|3 M-12 L|$. It's easy to check that $V$ is given by the form

$$
\alpha_{9} x_{1}^{3}+\alpha_{6} x_{1}^{2} x_{2}+\alpha_{5} x_{1}^{2} x_{3}+\alpha_{2}^{1} x_{1}^{2} x_{4}+\alpha_{3} x_{1} x_{2}^{2}+\alpha_{2}^{2} x_{1} x_{2} x_{3}+\alpha_{1} x_{1} x_{3}^{2}+\alpha_{0} x_{2}^{3} .
$$

After a coordinate change similar to one considered in Example 4.12 the singularity at $O$ is given by the zeroes of the polynomial

$$
x^{3}+x^{2} y+x^{2} z+x^{2}+x y^{2}+x y z+x z^{2}+y^{3} .
$$

If we put $\operatorname{wt}(x)=6, \operatorname{wt}(y)=4$ and $\operatorname{wt}(z)=3$, it is easy to check that the term of the lowest weight defines an isolated singularity. The weights coincide with the weights of $\mathbb{E}_{6}$. Then by Theorem 2.10 in the neighbourhood of $O$ the singularity is isomorphic to $\mathbb{E}_{6} \times \mathbb{C}$, and by Corollary 2.5 the singularities of $V$ are canonical.

$\left(T_{56}\right)$ : A divisor $V \subset \mathbb{F}(7,5,3,0)$ from the linear system $|3 M-13 L|$. It's easy to check that $V$ is given by the form

$$
\begin{aligned}
\alpha_{8} x_{1}^{3}+\alpha_{6} x_{1}^{2} x_{2}+\alpha_{4}^{1} x_{1}^{2} x_{3}+\alpha_{1}^{1} x_{1}^{2} x_{4}+ & \alpha_{4}^{2} x_{1} x_{2}^{2}+ \\
& +\alpha_{2}^{1} x_{1} x_{2} x_{3}+\alpha_{0} x_{1} x_{3}^{2}+\alpha_{2}^{2} x_{2}^{3}+\alpha_{1}^{2} x_{2}^{2} x_{3} .
\end{aligned}
$$

After a coordinate change similar to one considered in Example 4.12 the singularity at $O$ is given by the zeroes of the polynomial

$$
x^{3}+x^{2} y+x^{2} z+x^{2}+x y^{2}+x y z+x z^{2}+y^{3}+y^{2} z .
$$

Put $\operatorname{wt}(x)=4, \operatorname{wt}(y)=3$ and $\operatorname{wt}(z)=2$. It is quite easy to check that the term of the lowest weight defines an isolated singularity. The weights coincide with the weights of $\mathbb{D}_{5}$. Then by Theorem 2.10 in the neighbourhood of $O$ the singularity is isomorphic to $\mathbb{D}_{5} \times \mathbb{C}$, and by Corollary 2.5 the singularities of $V$ are canonical.

$\left(T_{57}\right)$ : A divisor $V \subset \mathbb{F}(7,5,4,0)$ from the linear system $|3 M-14 L|$. It's easy to check that $V$ is given by the form

$$
\begin{aligned}
\alpha_{7} x_{1}^{3}+\alpha_{5} x_{1}^{2} x_{2}+\alpha_{4} x_{1}^{2} x_{3}+\alpha_{0}^{1} x_{1}^{2} x_{4}+ & \alpha_{3} x_{1} x_{2}^{2}+ \\
& +\alpha_{2} x_{1} x_{2} x_{3}+\alpha_{1}^{1} x_{1} x_{3}^{2}+\alpha_{1}^{2} x_{2}^{3}+\alpha_{0}^{2} x_{2}^{2} x_{3} .
\end{aligned}
$$

After a coordinate change similar to one considered in Example 4.12 the singularity at $O$ is given by the zeroes of the polynomial

$$
x^{3}+x^{2} y+x^{2} z+x^{2}+x y^{2}+x y z+x z^{2}+y^{3}+y^{2} z .
$$

Let $\operatorname{wt}(x)=4, \operatorname{wt}(y)=3$ and $\operatorname{wt}(z)=2$. It is easy to check that the term of the lowest weight defines an isolated singularity. Moreover, the weights coincide with the weights of $\mathbb{D}_{5}$. Then by Theorem 2.10 in the neighbourhood of $O$ the singularity is isomorphic to $\mathbb{D}_{5} \times \mathbb{C}$, and by Corollary 2.5 the singularities of $V$ are canonical.

$\left(T_{58}\right)$ : A divisor $V \subset \mathbb{F}(7,5,4,1)$ from the linear system $|3 M-15 L|$. It's easy to check that $V$ is given by the form

$$
\alpha_{6} x_{1}^{3}+\alpha_{4} x_{1}^{2} x_{2}+\alpha_{3} x_{1}^{2} x_{3}+\alpha_{0}^{1} x_{1}^{2} x_{4}+\alpha_{2} x_{1} x_{2}^{2}+\alpha_{1} x_{1} x_{2} x_{3}+\alpha_{0}^{2} x_{1} x_{3}^{2}+\alpha_{0}^{3} x_{2}^{3} .
$$


After a coordinate change similar to one considered in Example 4.12 the singularity at $O$ is given by the zeroes of the polynomial

$$
x^{3}+x^{2} y+x^{2} z+x^{2}+x y^{2}+x y z+x z^{2}+y^{3} .
$$

$\operatorname{Put} \operatorname{wt}(x)=6, \operatorname{wt}(y)=4$ and $\operatorname{wt}(z)=3$. It is easy to check that the term of the lowest weight defines an isolated singularity. The weights coincide with the weights of $\mathbb{E}_{6}$. Then by Theorem 2.10 in the neighbourhood of $O$ the singularity is isomorphic to $\mathbb{E}_{6} \times \mathbb{C}$, and by Corollary 2.5 the singularities of $V$ are canonical.

$\left(T_{59}\right)$ : A divisor $V \subset \mathbb{F}(8,4,2,0)$ from the linear system $|3 M-12 L|$. It's easy to check that $V$ is given by the form

$$
\begin{aligned}
\alpha_{12} x_{1}^{3}+\alpha_{8} x_{1}^{2} x_{2}+\alpha_{6} x_{1}^{2} x_{3}+\alpha_{4}^{1} x_{1}^{2} x_{4} & +\alpha_{4}^{2} x_{1} x_{2}^{2}+ \\
& +\alpha_{2}^{1} x_{1} x_{2} x_{3}+\alpha_{2}^{2} x_{1} x_{2} x_{4}+\alpha_{0}^{1} x_{1} x_{3}^{2}+\alpha_{0}^{2} x_{2}^{3}
\end{aligned}
$$

After a coordinate change similar to one considered in Example 4.12 the singularity at $O$ is given by the zeroes of the polynomial

$$
x^{3}+x^{2} y+x^{2} z+x^{2}+x y^{2}+x y z+x y+x z^{2}+y^{3} .
$$

It is easy to check that this singularity is isolated. But here we can not choose "good" weights, so we should resolve the singularity at $O$. After three blow ups we obtain a smooth surface and exceptional curves will form a Dynkin diagram $\mathbb{A}_{5}$. So in the neighbourhood of $O$ the singularity is isomorphic to $\mathbb{A}_{5} \times \mathbb{C}$, and by Corollary 2.5 the singularities of $V$ are canonical.

$\left(T_{60}\right)$ : A divisor $V \subset \mathbb{F}(8,5,3,0)$ from the linear system $|3 M-14 L|$. It's easy to check that $V$ is given by the form

$$
\alpha_{10} x_{1}^{3}+\alpha_{7} x_{1}^{2} x_{2}+\alpha_{5} x_{1}^{2} x_{3}+\alpha_{2}^{1} x_{1}^{2} x_{4}+\alpha_{4} x_{1} x_{2}^{2}+\alpha_{2}^{2} x_{1} x_{2} x_{3}+\alpha_{0} x_{1} x_{3}^{2}+\alpha_{1} x_{2}^{3} .
$$

After a coordinate change similar to one considered in Example 4.12 the singularity at $O$ is given by the zeroes of the polynomial

$$
x^{3}+x^{2} y+x^{2} z+x^{2}+x y^{2}+x y z+x z^{2}+y^{3} .
$$

$\operatorname{Put} \operatorname{wt}(x)=6, \operatorname{wt}(y)=4$ and $\operatorname{wt}(z)=3$. The term of the lowest weight defines a singularity that is isolated. The weights coincide with the weights of $\mathbb{E}_{6}$. Then by Theorem 2.10 in the neighbourhood of $O$ the singularity is isomorphic to $\mathbb{E}_{6} \times \mathbb{C}$, and by Corollary 2.5 the singularities of $V$ are canonical.

$\left(T_{61}\right)$ : A divisor $V \subset \mathbb{F}(8,5,4,0)$ from the linear system $|3 M-15 L|$. It's easy to check that $V$ is given by the form

$$
\alpha_{9} x_{1}^{3}+\alpha_{6} x_{1}^{2} x_{2}+\alpha_{5} x_{1}^{2} x_{3}+\alpha_{1}^{1} x_{1}^{2} x_{4}+\alpha_{3} x_{1} x_{2}^{2}+\alpha_{2} x_{1} x_{2} x_{3}+\alpha_{1}^{2} x_{1} x_{3}^{2}+\alpha_{0} x_{2}^{3} .
$$

After a coordinate change similar to one considered in Example 4.12 the singularity at $O$ is given by the zeroes of the polynomial

$$
x^{3}+x^{2} y+x^{2} z+x^{2}+x y^{2}+x y z+x z^{2}+y^{3} .
$$

Let $\operatorname{wt}(x)=6, \operatorname{wt}(y)=4$ and $\operatorname{wt}(z)=3$. Then it is not hard to check that the term of the lowest weight defines an isolated singularity. The weights coincide with the weights of $\mathbb{E}_{6}$. Then by Theorem 2.10 in the neighbourhood of $O$ the singularity is isomorphic to $\mathbb{E}_{6} \times \mathbb{C}$, and by Corollary 2.5 the singularities of $V$ are canonical. 
$\left(T_{62}\right)$ : A divisor $V \subset \mathbb{F}(8,6,4,0)$ from the linear system $|3 M-16 L|$. It's easy to check that $V$ is given by the form

$$
\alpha_{8} x_{1}^{3}+\alpha_{6} x_{1}^{2} x_{2}+\alpha_{4}^{1} x_{1}^{2} x_{3}+\alpha_{0}^{1} x_{1}^{2} x_{4}+\alpha_{4}^{2} x_{1} x_{2}^{2}+\alpha_{2}^{1} x_{1} x_{2} x_{3}+\alpha_{0}^{2} x_{1} x_{3}^{2}+\alpha_{2}^{2} x_{2}^{3}+\alpha_{0}^{3} x_{2}^{2} x_{3} .
$$

After a coordinate change similar to one considered in Example 4.12 the singularity at $O$ is given by the zeroes of the polynomial

$$
x^{3}+x^{2} y+x^{2} z+x^{2}+x y^{2}+x y z+x z^{2}+y^{3}+y^{2} z .
$$

$\operatorname{Put} \operatorname{wt}(x)=4, \operatorname{wt}(y)=3$ and $\operatorname{wt}(z)=2$. Now the term of the lowest weight defines a singularity that is isolated. Moreover, the weights coincide with the weights of $\mathbb{D}_{5}$. Then by Theorem 2.10 in the neighbourhood of $O$ the singularity is isomorphic to $\mathbb{D}_{5} \times \mathbb{C}$, and by Corollary 2.5 the singularities of $V$ are canonical.

$\left(T_{63}\right):$ A divisor $V \subset \mathbb{F}(9,5,3,0)$ from the linear system $|3 M-15 L|$. It's easy to check that $V$ is given by the form

$$
\alpha_{12} x_{1}^{3}+\alpha_{8} x_{1}^{2} x_{2}+\alpha_{6} x_{1}^{2} x_{3}+\alpha_{3} x_{1}^{2} x_{4}+\alpha_{4} x_{1} x_{2}^{2}+\alpha_{2} x_{1} x_{2} x_{3}+\alpha_{0}^{1} x_{1} x_{3}^{2}+\alpha_{0}^{2} x_{2}^{3} .
$$

After a coordinate change similar to one considered in Example 4.12 the singularity at $O$ is given by the zeroes of the polynomial

$$
x^{3}+x^{2} y+x^{2} z+x^{2}+x y^{2}+x y z+x z^{2}+y^{3} .
$$

Let $\operatorname{wt}(x)=6, \operatorname{wt}(y)=4$ and $\operatorname{wt}(z)=3$. Then the term of the lowest weight defines an isolated singularity. The weights coincide with the weights of $\mathbb{E}_{6}$. Then by Theorem 2.10 in the neighbourhood of $O$ the singularity is isomorphic to $\mathbb{E}_{6} \times \mathbb{C}$, and by Corollary 2.5 the singularities of $V$ are canonical.

$\left(T_{64}\right)$ : A divisor $V \subset \mathbb{F}(9,6,4,0)$ from the linear system $|3 M-17 L|$. It's easy to check that $V$ is given by the form

$$
\alpha_{10} x_{1}^{3}+\alpha_{7} x_{1}^{2} x_{2}+\alpha_{5} x_{1}^{2} x_{3}+\alpha_{1}^{1} x_{1}^{2} x_{4}+\alpha_{4} x_{1} x_{2}^{2}+\alpha_{2} x_{1} x_{2} x_{3}+\alpha_{0} x_{1} x_{3}^{2}+\alpha_{1}^{2} x_{2}^{3} .
$$

After a coordinate change similar to one considered in Example 4.12 the singularity at $O$ is given by the zeroes of the polynomial

$$
x^{3}+x^{2} y+x^{2} z+x^{2}+x y^{2}+x y z+x z^{2}+y^{3} .
$$

$\operatorname{Put} \operatorname{wt}(x)=6, \operatorname{wt}(y)=4$ and $\operatorname{wt}(z)=3$. It is easy to check that the term of the lowest weight defines an isolated singularity. Moreover, the weights coincide with the weights of $\mathbb{E}_{6}$. Then by Theorem 2.10 in the neighbourhood of $O$ the singularity is isomorphic to $\mathbb{E}_{6} \times \mathbb{C}$, and by Corollary 2.5 the singularities of $V$ are canonical.

$\left(T_{65}\right)$ : A divisor $V \subset \mathbb{F}(9,6,5,0)$ from the linear system $|3 M-18 L|$. It's easy to check that $V$ is given by the form

$$
\alpha_{9} x_{1}^{3}+\alpha_{6} x_{1}^{2} x_{2}+\alpha_{5} x_{1}^{2} x_{3}+\alpha_{0}^{1} x_{1}^{2} x_{4}+\alpha_{3} x_{1} x_{2}^{2}+\alpha_{2} x_{1} x_{2} x_{3}+\alpha_{1} x_{1} x_{3}^{2}+\alpha_{0}^{2} x_{2}^{3} .
$$

After a coordinate change similar to one considered in Example 4.12 the singularity at $O$ is given by the zeroes of the polynomial

$$
x^{3}+x^{2} y+x^{2} z+x^{2}+x y^{2}+x y z+x z^{2}+y^{3} .
$$

Let $\operatorname{wt}(x)=6, \operatorname{wt}(y)=4$ and $\operatorname{wt}(z)=3$. Then the term of the lowest weight defines a singularity that is isolated. The weights coincide with the weights of $\mathbb{E}_{6}$. 
Then by Theorem 2.10 in the neighbourhood of $O$ the singularity is isomorphic to $\mathbb{E}_{6} \times \mathbb{C}$, and by Corollary 2.5 the singularities of $V$ are canonical.

$\left(T_{66}\right):$ A divisor $V \subset \mathbb{F}(10,6,4,0)$ from the linear system $|3 M-18 L|$. It's easy to check that $V$ is given by the form

$$
\alpha_{12} x_{1}^{3}+\alpha_{8} x_{1}^{2} x_{2}+\alpha_{6} x_{1}^{2} x_{3}+\alpha_{2}^{1} x_{1}^{2} x_{4}+\alpha_{4} x_{1} x_{2}^{2}+\alpha_{2}^{2} x_{1} x_{2} x_{3}+\alpha_{0}^{1} x_{1} x_{3}^{2}+\alpha_{0}^{2} x_{2}^{3} .
$$

After a coordinate change similar to one considered in Example 4.12 the singularity at $O$ is given by the zeroes of the polynomial

$$
x^{3}+x^{2} y+x^{2} z+x^{2}+x y^{2}+x y z+x z^{2}+y^{3} .
$$

If we put $\operatorname{wt}(x)=6, \operatorname{wt}(y)=4$ and $\operatorname{wt}(z)=3$, it is easy to check that the term of the lowest weight defines a singularity that is isolated. The weights coincide with the weights of $\mathbb{E}_{6}$. Then by Theorem 2.10 in the neighbourhood of $O$ the singularity is isomorphic to $\mathbb{E}_{6} \times \mathbb{C}$, and by Corollary 2.5 the singularities of $V$ are canonical.

$\left(T_{67}\right):$ A divisor $V \subset \mathbb{F}(10,7,5,0)$ from the linear system $|3 M-20 L|$. It's easy to check that $V$ is given by the form

$$
\alpha_{10} x_{1}^{3}+\alpha_{7} x_{1}^{2} x_{2}+\alpha_{5} x_{1}^{2} x_{3}+\alpha_{0}^{1} x_{1}^{2} x_{4}+\alpha_{4} x_{1} x_{2}^{2}+\alpha_{2} x_{1} x_{2} x_{3}+\alpha_{0}^{2} x_{1} x_{3}^{2}+\alpha_{1} x_{2}^{3} .
$$

After a coordinate change similar to one considered in Example 4.12 the singularity at $O$ is given by the zeroes of the polynomial

$$
x^{3}+x^{2} y+x^{2} z+x^{2}+x y^{2}+x y z+x z^{2}+y^{3} .
$$

Let us put $\operatorname{wt}(x)=6, \operatorname{wt}(y)=4$ and $\operatorname{wt}(z)=3$. It is easy to check that the term of the lowest weight defines an isolated singularity. The weights coincide with the weights of Du Val singularitiy $\mathbb{E}_{6}$. Then by Theorem 2.10 in the neighbourhood of $O$ the singularity is isomorphic to $\mathbb{E}_{6} \times \mathbb{C}$, and by Corollary 2.5 the singularities of $V$ are canonical.

$\left(T_{68}\right)$ : A divisor $V \subset \mathbb{F}(11,7,5,0)$ from the linear system $|3 M-21 L|$. It's easy to check that $V$ is given by the form

$$
\alpha_{12} x_{1}^{3}+\alpha_{8} x_{1}^{2} x_{2}+\alpha_{6} x_{1}^{2} x_{3}+\alpha_{1}^{1} x_{1}^{2} x_{4}+\alpha_{4} x_{1} x_{2}^{2}+\alpha_{2} x_{1} x_{2} x_{3}+\alpha_{0} x_{1} x_{3}^{2}+\alpha_{1}^{2} x_{2}^{3} .
$$

After a coordinate change similar to one considered in Example 4.12 the singularity at $O$ is given by the zeroes of the polynomial

$$
x^{3}+x^{2} y+x^{2} z+x^{2}+x y^{2}+x y z+x z^{2}+y^{3} .
$$

$\operatorname{Put} \operatorname{wt}(x)=6, \operatorname{wt}(y)=4$ and $\operatorname{wt}(z)=3$. One can easyly check that the term of the lowest weight defines an isolated singularity. Moreover, the weights coincide with the weights of $\mathbb{E}_{6}$. Then by Theorem 2.10 in the neighbourhood of $O$ the singularity is isomorphic to $\mathbb{E}_{6} \times \mathbb{C}$, and by Corollary 2.5 the singularities of $V$ are canonical.

( $\left.T_{69}\right)$ : A divisor $V \subset \mathbb{F}(12,8,6,0)$ from the linear system $|3 M-24 L|$. It's easy to check that $V$ is given by the form

$$
\alpha_{12} x_{1}^{3}+\alpha_{8} x_{1}^{2} x_{2}+\alpha_{6} x_{1}^{2} x_{3}+\alpha_{0}^{1} x_{1}^{2} x_{4}+\alpha_{4} x_{1} x_{2}^{2}+\alpha_{2} x_{1} x_{2} x_{3}+\alpha_{0}^{2} x_{1} x_{3}^{2}+\alpha_{0}^{3} x_{2}^{3} .
$$


After a coordinate change similar to one considered in Example 4.12 the singularity at $O$ is given by the zeroes of the polynomial

$$
x^{3}+x^{2} y+x^{2} z+x^{2}+x y^{2}+x y z+x z^{2}+y^{3} .
$$

$\operatorname{Put} \operatorname{wt}(x)=6, \operatorname{wt}(y)=4$ and $\operatorname{wt}(z)=3$. Then the term of the lowest weight defines a singularity that is isolated. The weights coincide with the weights of $\mathbb{E}_{6}$. Then by Theorem 2.10 in the neighbourhood of $O$ the singularity is isomorphic to $\mathbb{E}_{6} \times \mathbb{C}$, and by Corollary 2.5 the singularities of $V$ are canonical.

Therefore, Theorem 1.6 is proved. The proof of Theorem 1.6 gives a description of the possible singularities of the 3 -folds $T_{j}$. The sufficiently general 3 -fold $T_{j}$ is smooth for $j \in\{1,2,5,8,14\}$ and has only ordinary double points for $j \in\{4,7,11,16\}$. In the former case the 3 -folds $T_{j}$ are well known (see [58, 68]). The 3 -fold $T_{j}$ has non-isolated singularities for $j \in\{6,9,13,15,17,21,24,25,26,28,32,34,36,40,41,44,45,50,58\}$. In all other cases $T_{j}$ has at least one non-cDV point.

\section{RATiOnALity AND NON-RATiONALiTy.}

In this section we will prove Proposition 1.10, Let $H_{i}$ and $T_{j}$ be Fano 3 -folds from Theorem [1.5 and 1.6 respectively. Then the non-rationality of the sufficiently general 3-folds $H_{1}, H_{2}, H_{3}, H_{4}, H_{6}, T_{1}, T_{2}, T_{7}, T_{8}$ are definitely implied (see Remark [1.8 and Example 1.11) by 67, [21, [8], [118, [59], 112, [113, [114, 114, [19, 13., 6], [115], [96], [97], 20], 98, 445, 444, [22], 78, 23], 46], 86], 47], [18. On the other hand, it is clear that the 3 -folds $H_{9}, T_{5}$ and $T_{14}$ are rational (see Remark 1.8).

We may assume that $i \notin\{1,2,3,4,6,9\}$ and $j \notin\{1,2,5,7,8,14\}$. Therefore, the 3fold $H_{i}$ is naturally birational to a del Pezzo fibration $\tau: Y_{i} \rightarrow \mathbb{P}^{1}$ of degree 2 with canonical Gorenstein singularities (see Theorem 1.5) and $T_{j}$ is naturally birational to a del Pezzo fibration $\psi: V_{j} \rightarrow \mathbb{P}^{1}$ of degree 3 with canonical Gorenstein singularities (see Theorem (1.6). Let $\bar{Y}_{i}$ and $\bar{V}_{j}$ be generic fibers of $\tau$ and $\psi$ respectively. Then $\bar{Y}_{i}$ and $\bar{V}_{j}$ are del Pezzo surfaces with Du Val singularities defined over the field $\mathbb{C}(x)$.

Remark 5.1. The rationality of the surfaces $\bar{Y}_{i}$ and $\bar{V}_{j}$ over $\mathbb{C}(x)$ implies the rationality of the 3 -folds $Y_{i}$ and $V_{j}$ respectively.

The del Pezzo surfaces $\bar{Y}_{i}$ and $\bar{V}_{j}$ always have a $\mathbb{C}(x)$-point due to Theorem 2.24 (moreover, the sets of $\mathbb{C}(x)$-points of $\bar{Y}_{i}$ and $\bar{V}_{j}$ are huge by Theorem 2.25).

Lemma 5.2. Let $S$ be a del Pezzo surface of degree 3 with Du Val singularities defined over an arbitrary perfect field $\mathbb{F}$ such that the set $\operatorname{Sing}(S)$ contains an $\mathbb{F}$-point $O \in S$. Then $S$ is rational over $\mathbb{F}$.

Proof. The surface $S$ is a cubic hypersurface in $\mathbb{P}^{3}$ (see [80, [82, [65], [75]). Thus, the projection from $O$ gives a birational map to $\mathbb{P}^{2}$.

Therefore, the proof of Theorem 1.6 together with Lemma 5.2 imply the rationality of the 3 -fold $T_{j}$ for $j \in\{10,12,13,17, \ldots, 24,26, \ldots, 69\}$.

Lemma 5.3. Let $S$ be a del Pezzo surface of degree 2 with Du Val singularities defined over an arbitrary perfect field $\mathbb{F}$ such that the set $\operatorname{Sing}(S)$ contains an $\mathbb{F}$-point $O \in S$, which is of one of the following types: $\mathbb{E}_{6}, \mathbb{E}_{7}, \mathbb{D}_{n}$ or $\mathbb{A}_{k}$ for $n \geq 5$ and $k \geq 7$. Then $S$ is rational over $\mathbb{F}$. 
Proof. Let $f: W \rightarrow S$ be a minimal resolution of singularities of $S$ and $E=f^{-1}(O) \subset W$ be a connected curve defined over the field $\mathbb{F}$. Then $K_{W} \sim f^{*}\left(K_{S}\right)$. In particular, $W$ is a weak del Pezzo surface (see [26]) of degree 2, the curve $E$ is $\operatorname{Gal}(\overline{\mathbb{F}} / \mathbb{F})$-invariant and the components of $E$ defined over $\overline{\mathbb{F}}$ must split into disjoint $\mathrm{Gal}(\overline{\mathbb{F}} / \mathbb{F})$-orbits. However, the irreducible components of $E$ forms the graph of $\mathbb{E}_{6}, \mathbb{E}_{7}, \mathbb{D}_{n}$ or $\mathbb{A}_{k}$ for $n \geq 5$ and $k \geq 7$. Therefore, the curve $E$ splits into at least 3 possibly reducible curves defined over $\mathbb{F}$. The negativity of the intersection form of the irreducible components of the curve $E$ (see [5]) implies that the rank of $\operatorname{Pic}(W)$ is at least 5 .

There is a birational morphism $g: W \rightarrow U$ defined over $\mathbb{F}$ such that $U$ is minimal (see [57], 87], 65]), i.e. no curve on $U$ can be contracted to a smooth point. Moreover, the rank of $\operatorname{Pic}(U)$ is at most 2 by Theorem 2.22 . Therefore $K_{U}^{2} \geq K_{W}^{2}+3=5$. Thus, the surface $U$ is rational over $\mathbb{F}$ by Theorem 2.23 .

The proof of Theorem 1.5 together with Lemma 5.3 imply rationality of $H_{i}$ for $i \in$ $\{22,26,28,29,31, \ldots, 47\}$.

Remark 5.4. The non-rationality of the surfaces $\bar{Y}_{i}$ and $\bar{V}_{j}$ over $\mathbb{C}(x)$ does not imply the non-rationality of the 3 -folds $H_{i}$ and $T_{j}$ respectively. However, we believe that the rough method we used can be applied to the proof of non-rationality of many 3 -folds $H_{i}$ in many remaining cases. For example, one can try to use the proofs of Theorems 1.5] and [1.6 to get more delicate description of the geometry of the surfaces $\bar{Y}_{i}$ and $\bar{V}_{j}$ and use afterwards the results of [83], [116] and 65].

Proposition 5.5. Let $X$ be a sufficiently general 3-fold $T_{3}$ from Theorem 1.6. Then $X$ is non-rational.

Proof. Let $U=\operatorname{Proj}\left(\mathcal{O}_{\mathbb{P}^{2}}(2) \oplus \mathcal{O}_{\mathbb{P}^{2}} \oplus \mathcal{O}_{\mathbb{P}^{2}}\right), f: U \rightarrow \mathbb{P}^{2}$ be a natural projection, $T$ is tautological line bundle on $U$ and $F=f^{*}\left(\mathcal{O}_{\mathbb{P}^{2}}(1)\right)$. Then $X$ is an anticanonical image of a sufficiently general divisor $V \in|2 T+F|$. The 3 -fold $V$ is smooth by the Bertini theorem. Moreover, the Lefschetz theorem (see 9], 3]) implies $\operatorname{Pic}(V) \cong \mathbb{Z} \oplus \mathbb{Z}$.

Let $g: V \rightarrow \mathbb{P}^{2}$ be a restriction of the projection $f: U \rightarrow \mathbb{P}^{2}$. Then $g$ is a conic bundle. Let $\Delta$ be a degeneration divisor of $g$ and $Y$ be a sufficiently general surface in the linear system $\left|g^{*}\left(\mathcal{O}_{\mathbb{P}^{2}}(1)\right)\right|$. Then $Y$ is smooth and $K_{Y}^{2}=1$ by the adjunction formula. Therefore, the conic bundle $\left.g\right|_{Y}$ has 7 reducible fibers. Thus, the degree of $\Delta \subset \mathbb{P}^{2}$ is 7 (see $§ 3.5$ in [10]) and $V$ is non-rational by Theorem 2.16.

Proposition 5.6. Let $X$ be a sufficiently general ${ }^{2}$ 3-fold $H_{5}$ from Theorem [1.5. Then $X$ is non-rational.

Proof. The 3-fold $X$ is an anticanonical model of a smooth weak Fano 3 -fold $V$, such that there is a double cover $\pi: V \rightarrow U=\operatorname{Proj}\left(\mathcal{O}_{\mathbb{P}^{1}}(2) \oplus \mathcal{O}_{\mathbb{P}^{1}}(1) \oplus \mathcal{O}_{\mathbb{P}^{1}}\right)$ branched over a divisor $D \in|4 M-2 L|$, where $M$ is a tautological line bundle on $U$ and $L$ is a fiber of the natural projection of $U$ to $\mathbb{P}^{1}$. In the bihomogeneous coordinates (see Proposition 2.19) the divisor $D$ can be given by the zeroes of the bihomogeneous polynomial

$$
\begin{aligned}
\alpha_{6} x_{1}^{4}+\alpha_{5} x_{1}^{3} x_{2}+\alpha_{4}^{1} x_{1}^{3} x_{3} & +\alpha_{4}^{2} x_{1}^{2} x_{2}^{2}+\alpha_{3}^{1} x_{1}^{2} x_{2} x_{3}+\alpha_{2}^{1} x_{1}^{2} x_{3}^{2}+\alpha_{3}^{2} x_{1} x_{2}^{3}+ \\
& +\alpha_{2}^{2} x_{1} x_{2}^{2} x_{3}+\alpha_{1}^{1} x_{1} x_{2} x_{3}^{2}+\alpha_{0}^{1} x_{1} x_{3}^{3}+\alpha_{2}^{3} x_{2}^{4}+\alpha_{1}^{2} x_{2}^{3} x_{3}+\alpha_{0}^{2} x_{2}^{2} x_{3}^{2},
\end{aligned}
$$

where $\alpha_{d}^{i}=\alpha_{d}^{i}\left(t_{1}, t_{2}\right)$ is a homogeneous polynomial of degree $d$.

\footnotetext{
${ }^{2} \mathrm{~A}$ complement to a countable union of Zariski closed subsets in the moduli.
} 
Consider a double cover $\chi: Y \rightarrow U$ branched over a sufficiently general divisor $\Delta \subset U$ which is given by the zeroes of the same bihomogeneous polynomial as $D$ with the only exception that $\alpha_{0}^{1}=0$. Then $Y$ is not smooth, because $\Delta$ has singularities along the curve $Y_{3} \subset U$ given by $x_{1}=x_{2}=0$, which is the smallest negative subscroll of $U$ (see Proposition 2.19). Then $\Delta \subset U$ can be considered as a sufficiently general element of the linear subsystem in $|4 M-2 L|$ of divisors with singularities along $Y_{3}$. The Bertini theorem implies the smoothness of $\Delta$ outside of the curve $Y_{3}$.

Let $C=\chi^{-1}\left(Y_{3}\right)$. Then the 3 -fold $Y$ has singularities of type $\mathbb{A}_{1} \times \mathbb{C}$ at the general point of the curve $C$. Moreover, the singularities of $Y$ at other points of the curve $C$ are locally isomorphic to the singularity

$$
x^{2}+y^{2}+z^{2} t=0 \subset \mathbb{C}^{4} \cong \operatorname{Spec}(\mathbb{C}[x, y, z, t]),
$$

where the local equation of the curve $C$ is $x=y=z=0$. The latter implies that we can resolve the singularities of $Y$ by means of the blow up $f: \tilde{Y} \rightarrow Y$ of the curve $C$.

Let $g: \tilde{U} \rightarrow U$ be a blow up of the curve $Y_{3} \subset U$. Then the diagram

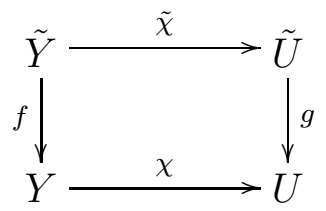

is commutative, where $\tilde{\chi}: \tilde{Y} \rightarrow \tilde{U}$ is a double cover. Let $E$ be an exceptional divisor of $g$. Then $\tilde{\chi}$ is branched over a divisor $g^{-1}(\Delta) \sim g^{*}(4 M-2 L)-2 E$.

The linear system $\left|g^{*}(M-L)-E\right|$ is free and the linear system $\left|g^{*}(M)-E\right|$ gives a $\mathbb{P}^{1}$-bundle $\tau: \tilde{U} \rightarrow \operatorname{Proj}\left(\mathcal{O}_{\mathbb{P}^{1}}(2) \oplus \mathcal{O}_{\mathbb{P}^{1}}(1) \cong \mathbb{F}_{1}\right.$. Therefore, the divisor $g^{-1}(\Delta) \sim$ $g^{*}(4 M-2 L)-2 E$ is ample on $\tilde{U}$ and the Lefschetz theorem (see [9], 3], 120]) implies $\operatorname{Pic}(\tilde{Y}) \cong \operatorname{Pic}(\tilde{U}) \cong \mathbb{Z}^{3}$ (see [19], 24], 25]).

In the case when the divisor $g^{-1}(\Delta)$ is ample on the variety $\tilde{U}$ the Lefschetz theorem (see [9], 3], 120]) implies $\operatorname{Pic}(\tilde{Y}) \cong \operatorname{Pic}(\tilde{U}) \cong \mathbb{Z}^{3}$ (. [19], 24], 25]). However, the divisor $g^{-1}(\Delta)$ is not ample, but it is nef and big. Indeed, the linear system $\left|g^{*}(M-L)-E\right|$ is free and the linear system $\left|g^{*}(M)-E\right|$ gives a $\mathbb{P}^{1}$-bundle $\tau: \tilde{U} \rightarrow \operatorname{Proj}\left(\mathcal{O}_{\mathbb{P}^{1}}(2) \oplus \mathcal{O}_{\mathbb{P}^{1}}(1)\right) \cong \mathbb{F}_{1}$. Therefore, the divisor $g^{-1}(\Delta) \sim g^{*}(4 M-2 L)-2 E$. Therefore instead of the Lefschetz theorem we can use the first part of the proof of Proposition 32 in $[10$, to get $\operatorname{Pic}(\tilde{Y}) \cong$ $\operatorname{Pic}(\tilde{U}) \cong \mathbb{Z}^{3}$.

Let $Y_{2} \subset U$ be the biggest negative subscroll (see Proposition [2.19), i.e. the surface given by $x_{1}=0$ in the bihomogeneous coordinates on $U$. Then $Y_{2} \cong \operatorname{Proj}\left(\mathcal{O}_{\mathbb{P}^{1}}(1) \oplus \mathcal{O}_{\mathbb{P}^{1}}\right)$. Let $S=g^{-1}\left(Y_{2}\right)$. Then $S \cong Y_{2}$ and the morphism $\tau$ contracts the surface $S$ to the exceptional section of $\mathbb{F}_{1}$.

The $\mathbb{P}^{1}$-bundle $\tau$ induces a conic bundle $\tilde{\tau}=\tau \circ \tilde{\chi}: \tilde{Y} \rightarrow \mathbb{F}_{1}$. Let $\tilde{S}=\tilde{\chi}^{-1}(S)$ and $Z \subset \tilde{Y}$ be a general fiber of the natural projection of $\tilde{Y}$ to $\mathbb{P}^{1}$. Then $Z$ is a smooth weak del Pezzo surface of degree 2, i.e. $-K_{Z}$ is nef and big and $K_{Z}^{2}=2$. Moreover, the morphism $\left.g \circ \tilde{\chi}\right|_{\tilde{S}}: \tilde{S} \rightarrow Y_{2}$ is a double cover branched over a divisor whose equation in the bihomogeneous coordinates is

$$
\alpha_{2}^{3}\left(t_{0}, t_{1}\right) x_{2}^{2}+\alpha_{1}^{2}\left(t_{0}, t_{1}\right) x_{2} x_{3}+\alpha_{0}^{2}\left(t_{0}, t_{1}\right) x_{3}^{2}=0,
$$

where $\alpha_{d}^{i}\left(t_{1}, t_{2}\right)$ is a homogeneous polynomial of degree $d$ from the bihomogeneous equation of $\Delta$. 
Let $\Xi \subset \mathbb{F}_{1}$ be a degeneration divisor of the conic bundle $\tilde{\tau}$. Then $\Xi \sim 6 s_{\infty}+a l$, where $s_{\infty}$ is an exceptional section of $\mathbb{F}_{1}, l$ is a fiber of the projection of $\mathbb{F}_{1}$ to $\mathbb{P}^{1}$ and $a \in \mathbb{Z}$. The structure of the morphism $\left.g \circ \tilde{\chi}\right|_{\tilde{S}}$ implies $s_{\infty} \not \subset \Xi$. Moreover, $s_{\infty} \cdot \Xi$ is equal to the number of the reducible fibers of the induced conic bundle $\left.\tilde{\tau}\right|_{\tilde{S}}$. The latter is easy to calculate from the bihomogeneous equation of the ramification divisor of $\left.g \circ \tilde{\chi}\right|_{\tilde{S}}$, i.e. the reducible fibers of $\left.\tilde{\tau}\right|_{\tilde{S}}$ correspond to the zeroes of the determinant $\left(\alpha_{1}^{2}\right)^{2}-4 \alpha_{0}^{2} \alpha_{2}^{3}$, i.e. $s_{\infty} \cdot \Xi=2$. Thus, $a=8$. Hence, $Y$ is non-rational by Theorem 2.16.

The 3-fold $Y$ is rationally connected (see 75]). Thus, the non-rationality of $Y$ implies that $Y$ is non-ruled as well. Therefore, the 3 -fold $V$ is non-ruled by Theorem 2.18, because $V$ is sufficiently general by assumption. Hence, $X$ is non-rational.

Proposition 5.7. Let $X$ be a sufficiently general ${ }^{3}$ 3-fold $H_{7}$ from Theorem 1.5 . Then $X$ is non-rational.

Proof. The 3 -fold $X$ is an anticanonical model of a weak Fano 3 -fold $V$, such that there is a double cover $\pi: V \rightarrow U=\operatorname{Proj}\left(\mathcal{O}_{\mathbb{P}^{1}}(2) \oplus \mathcal{O}_{\mathbb{P}^{1}}(2) \oplus \mathcal{O}_{\mathbb{P}^{1}}\right)$, branched over a divisor $D \in|4 M-4 L|$, where $M$ is a tautological line bundle on $U$ and $L$ is a fiber of the natural projection of $U$ to $\mathbb{P}^{1}$. In the bihomogeneous coordinates (see Proposition 2.19) the divisor $D$ can be given by the zeros of the bihomogeneous polynomial

$$
\begin{aligned}
\alpha_{4}^{1} x_{1}^{4}+\alpha_{4}^{2} x_{1}^{3} x_{2}+\alpha_{4}^{3} x_{1}^{2} x_{2}^{2}+\alpha_{4}^{4} x_{1} x_{2}^{3}+\alpha_{4}^{5} x_{2}^{4}+\alpha_{2}^{1} x_{1}^{3} x_{3}+\alpha_{2}^{2} x_{1}^{2} x_{2} x_{3}+ & \\
& +\alpha_{2}^{3} x_{1} x_{2}^{2} x_{3}+\alpha_{2}^{4} x_{2}^{3} x_{3}+\alpha_{0}^{1} x_{1}^{2} x_{3}^{2}+\alpha_{0}^{2} x_{1} x_{2} x_{3}^{2}+\alpha_{0}^{3} x_{2}^{2} x_{3}^{2},
\end{aligned}
$$

where $\alpha_{d}^{i}=\alpha_{d}^{i}\left(t_{1}, t_{2}\right)$ is a homogeneous polynomial of degree $d$.

The divisor $D$ has singularities along the curve $Y_{3} \subset U$ given by $x_{1}=x_{2}=0$. The generality of $X$ implies that $D \subset U$ is a general divisor in the linear system $|4 M-2 L|$. The Bertini theorem implies the smoothness of $D$ outside of the curve $Y_{3}$. The 3 -fold $V$ has singularities of type $\mathbb{A}_{1} \times \mathbb{C}$ at the general point of the curve $C=\chi^{-1}\left(Y_{3}\right)$. Moreover, we can resolve the singularities of $V$ by means of the blow up $f: \tilde{V} \rightarrow V$ of the curve $C$.

Let $g: \tilde{U} \rightarrow U$ be a blow up of the curve $Y_{3} \subset U$. Then the diagram

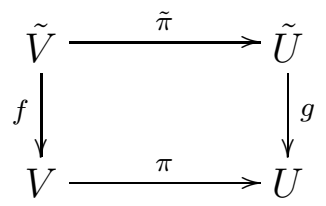

is commutative, where $\tilde{\pi}: \tilde{V} \rightarrow \tilde{U}$ is a double cover. Let $E$ be an exceptional divisor of $g$. Then $\tilde{\pi}$ is branched over a divisor $g^{-1}(D) \sim g^{*}(4 M-4 L)-2 E$. However, the linear system $\left|g^{*}(M-2 L)-E\right|$ is a free pencil whose image on $U$ is generated by divisors $x_{1}=0$ and $x_{2}=0$ in bihomogeneous coordinates. Therefore, the divisor $g^{-1}(D) \sim g^{*}(4 M-2 L)-2 E$ is nef and big on $\tilde{U}$. Now the first part of the proof of Proposition 32 in [10] (the stronger version of the Lefschetz theorem) implies $\operatorname{Pic}(\tilde{V}) \cong \operatorname{Pic}(\tilde{U}) \cong \mathbb{Z}^{3}$.

The linear system $\left|g^{*}(M-L)-E\right|$ is free as well and gives a $\mathbb{P}^{1}$-bundle $\tau: \tilde{U} \rightarrow$ $\operatorname{Proj}\left(\mathcal{O}_{\mathbb{P}^{1}}(2) \oplus \mathcal{O}_{\mathbb{P}^{1}}(2) \cong \mathbb{F}_{0}\right)$. In the bihomogeneous coordinates the rational map $\tau \circ g^{-1}$ is given by linear system on $U$ spanned by $\beta_{1}\left(t_{0}, t_{2}\right) x_{1}+\beta_{2}\left(t_{0}, t_{2}\right) x_{2}$, where $\beta_{i}\left(t_{0}, t_{2}\right)$ is a homogeneous polynomial of degree 1 .

The $\mathbb{P}^{1}$-bundle $\tau$ induces a conic bundle $\tilde{\tau}=\tau \circ \tilde{\pi}: \tilde{V} \rightarrow \mathbb{F}_{0}$. Let $\Delta \subset \mathbb{F}_{0}$ be a degeneration divisor of $\tilde{\tau}, L_{1}$ and $L_{2}$ be fibers of two projections of $\mathbb{F}_{0}$ to $\mathbb{P}^{1}$, such that

\footnotetext{
${ }^{3} \mathrm{~A}$ complement to a Zariski closed subset in the moduli.
} 
$\tau^{*}\left(L_{1}\right) \sim g^{*}(L)$ and $\tau^{*}\left(L_{2}\right) \sim g^{*}(M-2 L)-E$. Then $\Delta \sim n L_{1}+6 L_{2}$ for some $n \in \mathbb{Z}$. Moreover, elementary calculations (see the proof of Proposition [5.6) imply $n=4$. Hence, $V$ is non-rational by Theorem 2.16 .

Proposition 5.8. Let $X$ be a 3-fold $H_{8}$ from Theorem 1.5. Then $X$ is rational.

Proof. We can proceed as in the proof of Proposition [5.7 to get a conic bundle $\tilde{\tau}=\tau \circ \tilde{\pi}$ : $\tilde{V} \rightarrow \mathbb{P}^{1} \times \mathbb{P}^{1}$, where $\tilde{V}$ is birational to $X$. Moreover, we do not need to prove that the conic bundle $\tilde{\tau}$ is standard, i.e. that $\operatorname{Pic}(\tilde{V}) \cong \mathbb{Z}^{3}$. A simple calculation implies that the degeneration divisor $\Delta \subset \mathbb{P}^{1} \times \mathbb{P}^{1}$ of $\tilde{\tau}$ has bi-degree $(6,2)$. Now the rationality of $X$ is immediately implied by Theorems 2.24, 2.22] and 2.23,

Proposition 5.9. Let $X$ be a sufficiently general ${ }^{4}$ 3-fold $T_{4}$ from Theorem 1.6 . Then $X$ is non-rational.

Proof. The 3 -fold $X$ is an anticanonical image of a sufficiently general divisor $V \subset U=$ $\operatorname{Proj}\left(\mathcal{O}_{\mathbb{P}^{1}}(1) \oplus \mathcal{O}_{\mathbb{P}^{1}}(1) \oplus \mathcal{O}_{\mathbb{P}^{1}}(1) \oplus \mathcal{O}_{\mathbb{P}^{1}}\right)$ from the linear system $|3 M-L|$, where $M$ is a tautological line bundle on $U$ and $L$ is a fiber of the natural projection of $U$ to $\mathbb{P}^{1}$. Then in the bihomogeneous coordinates on $U$ the divisor $V$ is given by

$$
\begin{aligned}
\alpha_{2}^{1} x_{1}^{3}+\alpha_{2}^{2} x_{1}^{2} x_{2} & +\alpha_{2}^{3} x_{1}^{2} x_{3}+\alpha_{2}^{4} x_{1}^{2} x_{2}+\alpha_{2}^{5} x_{1} x_{2} x_{3}+\alpha_{2}^{6} x_{1} x_{3}^{2}+ \\
& +\alpha_{2}^{7} x_{2}^{3}+\alpha_{2}^{8} x_{2}^{2} x_{3}+\alpha_{2}^{9} x_{2} x_{3}^{2}+\alpha_{2}^{10} x_{3}^{3}+\alpha_{1}^{1} x_{1}^{2} x_{4}+\alpha_{1}^{2} x_{1} x_{2} x_{4}+ \\
& +\alpha_{1}^{3} x_{1} x_{3} x_{4}+\alpha_{1}^{4} x_{2}^{2} x_{4}+\alpha_{1}^{5} x_{2} x_{3} x_{4}+\alpha_{1}^{5} x_{3}^{2} x_{4}+\alpha_{0}^{1} x_{1} x_{4}^{2}+\alpha_{0}^{2} x_{2} x_{4}^{2}+\alpha_{0}^{3} x_{3} x_{4}^{2}=0
\end{aligned}
$$

where $\alpha_{d}^{i}=\alpha_{d}^{i}\left(t_{0}, t_{1}\right)$ is a homogeneous polynomial of degree $d$. The generality of $X$ implies the smoothness of $V$. Moreover, the anticanonical morphism $\phi_{\left|-K_{V}\right|}$ contracts a single curve $C \subset V$ given by the equations $x_{1}=x_{2}=x_{3}=0$ to an ordinary double point $O$ on $X$. The corresponding birational morphism $\phi_{|M|}$ maps the rational scroll $U$ to a cone $\bar{U}$ over $\mathbb{P}^{1} \times \mathbb{P}^{2}$ with a vertex $O$.

The 3 -fold $X$ and the 4 -fold $\bar{U}$ are not $\mathbb{Q}$-factorial. Moreover, the birational morphisms $\phi_{\left|-K_{V}\right|}$ and $\phi_{|M|}$ are the $\mathbb{Q}$-factorializations of $X$ and $\bar{U}$ respectively (see [71]). There are other choices of the $\mathbb{Q}$-factorializations of $X$ and $\bar{U}$. Namely, there is scroll $\tilde{U}=$ $\operatorname{Proj}\left(\mathcal{O}_{\mathbb{P}^{2}}(1) \oplus \mathcal{O}_{\mathbb{P}^{2}}(1) \oplus \mathcal{O}_{\mathbb{P}^{2}}\right)$, and a birational morphism $\phi_{|T|}: \tilde{U} \rightarrow \bar{U}$, where $T$ is tautological line bundle on $\tilde{U}$. Moreover, the birational map $\phi_{|T|}^{-1} \circ \phi_{|M|}$ is an antiflip (see [72, 79]) in the curve $C \subset U$.

Let $Y \subset \tilde{U}$ be a proper transform of $X$ on the 4-fold $\tilde{U}$. Then $Y$ is a smooth weak Fano 3-fold and $Y \sim 2 T+F$ for $F=f^{*}\left(\mathcal{O}_{\mathbb{P}^{2}}(1)\right)$, where $f$ is a natural projection of $\tilde{U}$ to $\mathbb{P}^{2}$. Moreover, the 3 -fold $X$ is an anticanonical image of the 3 -fold $Y$ and the birational map $\phi_{\left|-K_{Y}\right|}^{-1} \circ \phi_{\left|-K_{V}\right|}$ is a simple flop in the curve $C \subset V$ induced by the antiflip $\phi_{|T|}^{-1} \circ \phi_{|M|}$. The Lefschetz theorem implies $\operatorname{Pic}(Y) \cong \mathbb{Z} \oplus \mathbb{Z}$.

Let $g: Y \rightarrow \mathbb{P}^{2}$ be a restriction of the projection $f: \tilde{U} \rightarrow \mathbb{P}^{2}$. Then $g$ is a conic bundle. Let $\Delta$ be a degeneration divisor of $g$. Then simple calculations (see the proof of Proposition [5.5) imply $\Delta \sim \mathcal{O}_{\mathbb{P}^{2}}(7)$ (see $\S 4.4 .1$ in [10]). Therefore, the 3 -fold $Y$ is non-rational by Theorem 2.16 (see also [8, 118]).

Proposition 5.10. Let $X$ be a sufficiently general ${ }^{5}$ 3-fold $T_{6}$ from Theorem 1.6 . Then $X$ is non-rational.

\footnotetext{
${ }^{4} \mathrm{~A}$ complement to a Zariski closed subset in the moduli.

${ }^{5} \mathrm{~A}$ complement to a Zariski closed subset in the moduli.
} 
Proof. The 3-fold $X$ is an anticanonical image of weak Fano 3 -fold $V$ that is a sufficiently general divisor on the rational scroll $U=\operatorname{Proj}\left(\mathcal{O}_{\mathbb{P}^{1}}(2) \oplus \mathcal{O}_{\mathbb{P}^{1}}(1) \oplus \mathcal{O}_{\mathbb{P}^{1}} \oplus \mathcal{O}_{\mathbb{P}^{1}}\right)$ lying in the linear system $|3 M-L|$, where $M$ is a tautological line bundle on $U$ and $L$ is a fiber of the natural projection of $U$ to $\mathbb{P}^{1}$. Then in the bihomogeneous coordinates on $U$ the 3 -fold $V$ is given by

$$
\begin{aligned}
& \alpha_{5} x_{1}^{3}+\alpha_{4} x_{1}^{2} x_{2}+\alpha_{3}^{1} x_{1}^{2} x_{3}+\alpha_{3}^{2} x_{1}^{2} x_{4}+\alpha_{3}^{3} x_{1} x_{2}^{2}+\alpha_{2}^{1} x_{1} x_{2} x_{3}+\alpha_{2}^{2} x_{1} x_{2} x_{4}+\alpha_{1}^{1} x_{1} x_{3}^{2}+ \\
& +\alpha_{1}^{2} x_{1} x_{3} x_{4}+\alpha_{1}^{3} x_{1} x_{4}^{2}+\alpha_{2}^{3} x_{2}^{3}+\alpha_{1}^{4} x_{2}^{2} x_{3}+\alpha_{1}^{5} x_{2}^{2} x_{4}+\alpha_{0}^{1} x_{2} x_{3}^{2}+\alpha_{0}^{2} x_{2} x_{3} x_{4}+\alpha_{0}^{3} x_{2} x_{4}^{2}=0,
\end{aligned}
$$

where $\alpha_{d}^{i}=\alpha_{d}^{i}\left(t_{0}, t_{1}\right)$ is a homogeneous polynomial of degree $d$. The 3 -fold $V$ contains a surface $Y_{3} \cong \mathbb{P}^{1} \times \mathbb{P}^{1}$ given by the equations $x_{1}=x_{2}=0$, which is the base locus of the linear system $|3 M-L|$. However, $V$ is smooth in the general point of $Y_{3}$. On the other hand, $V$ is always singular at the points where

$$
x_{1}=x_{2}=\alpha_{1}^{1} x_{3}^{2}+\alpha_{1}^{2} x_{3} x_{4}+\alpha_{1}^{3} x_{4}^{2}=\alpha_{0}^{1} x_{3}^{2}+\alpha_{0}^{2} x_{3} x_{4}+\alpha_{0}^{3} x_{4}^{2}=0,
$$

but the generality of the 3 -fold $V$ implies that these points are ordinary double points on $V$ and $V$ is smooth outside of them.

Let $g: \tilde{U} \rightarrow U$ be a blow up of the surface $Y_{3} \subset U, E$ be an exceptional divisor of $g$ and $\tilde{V}=g^{-1}(V) \subset \tilde{U}$. Then $\tilde{V} \sim g^{*}(3 M-L)-E, \tilde{V}$ is smooth and $\left.g\right|_{\tilde{V}}$ is a small resolution of the 3-fold $V$. On the other hand, the linear system $\left|g^{*}(M-L)-E\right|$ is free. Therefore, the divisor $\tilde{V}$ is nef and big on $\tilde{U}$. In the case of ample $\tilde{V}$ the Lefschetz theorem implies $\operatorname{Pic}(\tilde{V}) \cong \operatorname{Pic}(\tilde{U}) \cong \mathbb{Z}^{3}$. However, the divisor $\tilde{V}$ is not ample. Nevertheless, instead of the Lefschetz theorem we can use the arguments of the first part of the proof of Proposition 32 in [10] to get $\operatorname{Pic}(\tilde{V}) \cong \operatorname{Pic}(\tilde{U}) \cong \mathbb{Z}^{3}$.

The linear system $\left|g^{*}(M)-E\right|$ is free and gives a $\mathbb{P}^{2}$-bundle $\tau: \tilde{U} \rightarrow \operatorname{Proj}\left(\mathcal{O}_{\mathbb{P}^{1}}(2) \oplus\right.$ $\left.\left.\mathcal{O}_{\mathbb{P}^{1}}(1)\right) \cong \mathbb{F}_{1}\right)$. In the bihomogeneous coordinates the rational map $\tau \circ g^{-1}$ is given by a linear system on $U$ spanned by $\beta_{1}\left(t_{0}, t_{2}\right) x_{1}+\beta_{2}\left(t_{0}, t_{2}\right) x_{2}$, where $\beta_{i}\left(t_{0}, t_{2}\right)$ is a homogeneous polynomial of degree 1 .

The $\mathbb{P}^{2}$-bundle $\tau$ induces a conic bundle $\tilde{\tau}=\left.\tau\right|_{\tilde{V}}: \tilde{V} \rightarrow \mathbb{F}_{1}$. Let $\Delta \subset \mathbb{F}_{1}$ be a degeneration divisor of $\tilde{\tau}$. Then $\Delta \sim 5 s_{\infty}+a l$, where $s_{\infty}$ is an exceptional section of $\mathbb{F}_{1}$ and $l$ is a fiber of the projection of $\mathbb{F}_{1}$ to $\mathbb{P}^{1}$.

Let $s_{0}$ be a sufficiently general section of $\mathbb{F}_{1}$ such that $s_{0} \cap s_{\infty}=\emptyset$. Put $S=\tilde{\tau}^{-1}\left(s_{0}\right)$ and $B=\tau^{-1}\left(s_{0}\right)$. Then $S=B \cap \tilde{V} \subset B$ and $B$ is naturally isomorphic to the scroll $\operatorname{Proj}\left(\mathcal{O}_{\mathbb{P}^{1}}(2) \oplus \mathcal{O}_{\mathbb{P}^{1}} \oplus \mathcal{O}_{\mathbb{P}^{1}}\right)$. Moreover, $g(B) \cong B$ and $g(B) \cap V=g(S) \cup Y_{3}$. However, $Y_{3}$ is cut by $x_{1}=0$ on the scroll $g(B)$ and the scroll $g(B)$ is a general divisor in the linear system $|M-L|$. Therefore, we have $S \sim 2 T+F$ on the scroll $B$, where $T$ is a tautological line bundle on $B$ and $F$ is a fiber of the natural projection of $B$ to $\mathbb{P}^{1}$. The latter implies $K_{S}^{2}=1$. Therefore, $s_{0} \cdot \Delta=7$ and $a=7$. Hence, $\tilde{V}$ is non-rational by Theorem 2.16]

Proposition 5.11. Let $X$ be a 3-fold $T_{25}$ from Theorem 1.6. Then $X$ is rational.

Proof. We can repeat a construction of the conic bundle from Proposition 5.10 to get a conic bundle $\tilde{\tau}=\tilde{V} \rightarrow \mathbb{F}_{3}$, where $\tilde{V}$ is birational to $X$. However, we do not need the condition $\operatorname{Pic}(\tilde{V}) \cong \mathbb{Z}^{3}$ and the smoothness of $\tilde{V}$. Let $\Delta \subset \mathbb{F}_{3}$ be a degeneration divisor of $\tilde{\tau}$. Then elementary calculations imply $\Delta \cdot s_{0}=1$, where $s_{0}$ is a sufficiently general section on $\mathbb{F}_{3}$ that does not intersect an exceptional section on $\mathbb{F}_{3}$. Therefore, the 3 -fold $\tilde{V}$ is rational by Theorems $2.24,2.22$ and 2.23 . 
Proposition 5.12. Let $X$ be a sufficiently general ${ }^{6}$ 3-fold $T_{9}$ from Theorem 1.6. Then $X$ is non-rational.

Proof. We can repeat a construction of the conic bundle from the proof of Proposition 5.10 to get a conic bundle $\tilde{\tau}=\tilde{V} \rightarrow \mathbb{F}_{0} \cong \mathbb{P}^{1} \times \mathbb{P}^{1}$, such that $\tilde{V}$ is birational to $X$, the 3 -fold $\tilde{V}$ is smooth and $\operatorname{Pic}(\tilde{V}) \cong \mathbb{Z}^{3}$. Let $\Delta \subset \mathbb{F}_{0}$ be a degeneration divisor of $\tilde{\tau}$. Then elementary calculations (see the proof of Proposition [5.10) imply that $\Delta \subset \mathbb{P}^{1} \times \mathbb{P}^{1}$ has bi-degree $(5,4)$. Therefore, $\tilde{V}$ is non-rational by Theorem 2.16 .

Proposition 5.13. Let $X$ be 3-fold $T_{11}$ from Theorem [1.6. Then $X$ is rational.

Proof. We can repeat a construction of the conic bundle from the proof of Proposition 5.10 to get a conic bundle $\tilde{\tau}=\tilde{V} \rightarrow \mathbb{F}_{0} \cong \mathbb{P}^{1} \times \mathbb{P}^{1}$, such that $\tilde{V}$ is birational to $X$. However, we do not need to prove that $\operatorname{Pic}(\tilde{V}) \cong \mathbb{Z}^{3}$ and the 3 -fold $\tilde{V}$ is smooth. Let $\Delta \subset \mathbb{F}_{0}$ be a degeneration divisor of $\tilde{\tau}$. Then elementary calculations imply that $\Delta \subset \mathbb{P}^{1} \times \mathbb{P}^{1}$ has bi-degree $(5,2)$. Hence, we can consider a composition $\theta: \tilde{V} \rightarrow \mathbb{P}^{1}$ of the conic bundle $\tilde{\tau}$ with one of the projections of $\mathbb{P}^{1} \times \mathbb{P}^{1}$ to $\mathbb{P}^{1}$ such that the sufficiently general fiber of $\theta$ is a surface $S$ with $K_{S}^{2}=6$. Then the rationality of $\tilde{V}$ is implied by Theorems 2.24, 2.22 and 2.23 .

Therefore, we proved Proposition 1.10. The approach of proving the non-rationality of the 3 -fold $H_{i}$ and $T_{j}$ together with the degeneration technique (see [8, 118, [74]) can be used as a pattern for the proof of the non-rationality of many 3-folds fibered into del Pezzo surfaces of degree 2 and 3 (see [6], [99], [10, [16]).

\section{REFERENCES}

[1] V. Alexeev, Theorems about good divisors on log Fano varieties (case of index $r>n-2$ ), Lect. Note Math. 1479 (1991), 1-9.

[2] F. Ambro, Ladders on Fano varieties, J. Math. Sci. (New York) 94 (1999), 1126-1135.

[3] A. Andreotti, T. Frankel, The Lefschetz theorem on hyperplane sections, Ann. Math. 69 (1959), 713717.

[4] V.I. Arnold, S. M. Gusein-Zade, A. N. Varchenko, Singularities of differentiable maps I, Nauka, Moscow (1982).

[5] M. Artin, Some numerical criteria of contractability of curves on algebraic surfaces, Amer. J. Math. 84 (1962), 485-496.

[6] F. Bardelli, Polarized mixed Hodge structures: on irrationality of threefolds via degeneration, Ann. Mat. Pura et Appl. 137 (1984), 287-369.

[7] W. Barth, C. Peters, A. van de Ven, Compact complex surfaces, Ergebnisse der Mathematik und ihrer Grenzgebiete, Springer-Verlag (1984), Berlin-New York.

[8] A. Beauville, Varietes de Prym et jacobiennes intermediaires, Ann. Sci. Ecole Norm. Sup. 10 (1977), 309-391.

[9] R. Bott, On a theorem of Lefschetz, Mich. Math. J. 6 (1959), 211-216.

[10] G. Brown, A.Corti, F.Zucconi Birational geometry of 3-fold Mori fibre spaces, arXiv:math.AG/0307301 (2004).

[11] F. Campana, H. Flenner, Projective threefolds containing a smooth rational surface with ample normal bundle, J. Reine Angew. Math. 440 (1993), 77-98.

[12] I. Cheltsov, Singularities of 3-folds containing an ample effective divisor, which is a smooth surface of Kodaira dimension zero, Math. Notes, 594 (1996), 445-450.

[13] I. Cheltsov, 3-folds containing a divisor with numerically trivial canonical class, Russian Math. Survey, 511 (1996), 140-141.

[14] I. Cheltsov, Boundness of Fano 3-folds with an integer index, Math. Notes, 663 (1999), 445-451.

[15] I. Cheltsov, Double space with double line, preprint, MPIM (2003).

\footnotetext{
${ }^{6} \mathrm{~A}$ complement to a Zariski closed subset in the moduli.
} 
[16] I. Cheltsov, The degeneration method and the non-rationality of threefolds with a pencil of del Pezzo surfaces, Russian Math. Surveys (2004), to appear.

[17] I. Cheltsov, Non-rational nodal quartic threefolds, arXiv:math.AG/0405150 (2004).

[18] I. Cheltsov, J. Park, Sextic double solids, arXiv:math.AG/0404452 (2004).

[19] H. Clemens, Double solids, Adv. in Math. 47 (1983), 107-230.

[20] H. Clemens, The quartic double solid revisited, Complex geometry and Lie theory (Sundance, UT, 1989), Proc. Sympos. Pure Math. 53, (1991), 89-101.

[21] H. Clemens, P. Griffiths, The intermediate Jacobian of the cubic threefold, Ann. of Math. 95 (1972), $73-100$.

[22] A. Corti, Singularities of linear systems and 3-fold birational geometry, L.M.S. Lecture Note Series 281 (2000), 259-312.

[23] A. Corti, M. Mella, Birational geometry of terminal quartic 3-folds I, Amer. J. Math., to appear.

[24] S. Cynk, Defect of a nodal hypersurface, Manuscripta Math. 104 (2001), 325-331.

[25] S. Cynk, Cohomologies of a double covering of a non-singular algebraic 3-fold, Math. Z. 240 (2002), 731-743.

[26] M. Demazure, Surfaces de del Pezzo, Lecture Notes in Math. 777 Springer, Berlin-Heidelberg-New York (1989), 21-69.

[27] I. Dolgachev, Weighted projective varieties, Lecture Notes in Mathematics 956 (1982), 34-71.

[28] D. Eisenbud, J. Harris On varieties of minimal degree, Proc. Symp. Pure Math. 46 (1987), 3-13.

[29] R. Elkik, Rationalite des singularites canoniques, Invent. Math. 64 (1981), 1-6.

[30] F. Enriques, Sepra una involuzione non razionale de dello spacio, Rend. Acc. Lincei 21 (1912), 81-83.

[31] G. Fano, Sulle varietá algebriche a tre dimensioni aventi tutti i generi nulli, Atti Congr. Int. Bologna IV (1929), 115-121.

[32] G. Fano, Su alcune varietá algebriche a tre dimensioni a curve sezioni canoniche, Scritti Mat. offerti a L.Berzolari Ist. Mat. R. Univ. Pavia (1936), 329-349.

[33] G. Fano, Sulle varietá algebriche a tre dimensioni a curve sezioni canoniche, Mem. Accad. d'Italiana VIII (1937), 23-64.

[34] G. Fano, Su alcune varietá algebriche a tre dimensioni razionali, e aventi curve-sezioni canoniche, Comment. Math. Helv. 14 (1942), 202-211.

[35] A.R. Iano-Fletcher, Working with weighted complete intersections, L.M.S. Lecture Note Series 281 (2000), 101-173.

[36] T. Fujita, On the structure of polarized varieties with $\Delta$-genera zero, J. Fac. Sci. Univ. Tokyo Sec. IA 22 (1975), 103-115.

[37] T. Fujita, On singular Del Pezzo varieties, Lecture Notes in Math. 1417 (1990), 117-128.

[38] T. Fujita, Classification theories of polarized varieties, LMS Lecture Note Series 155 (1990).

[39] M. Furushima, Mukai-Umemura's example of a Fano 3-fold with genus 12 as a compactification of $\mathbb{C}^{12}$, Nagoya Math. Jour. 127 (1990), 145-165.

[40] M. Gizatullin, Rational G-surface, Izv. AN SSSR 16 (1981), 103-134.

[41] P. Griffiths, J. Harris, Principles of algebraic geometry, John Wiley, New York (1978).

[42] M. Grinenko, Birational automorphisms of three-dimensional double cone, Mat. Sbornik 189 (1998), $37-52$.

[43] M. Grinenko, Birational automorphisms of a three-dimensional dual quadric with the simplest singularity, Mat. Sbornik 189 (1998), 101-118.

[44] M. Grinenko, On the birational rigidity of some pencils of del Pezzo surfaces, Jour. Math. Sciences 102 (2000), 3933-3937.

[45] M. Grinenko, Birational properties of pencils of del Pezzo surfaces of degree 1 and 2, Mat. Sbornik 191 (2000), 633-653.

[46] M. Grinenko, On a double cone over the Veronese surface, Izv. MAth. 67 (2003), 421-438.

[47] M. Grinenko, Non-rationality of a three-dimensional Fano variety of index 2 and degree 1, Proc. Steklov Inst. 246 (2004), to appear.

[48] R. Hartshorne, Algebraic geometry, Graduate Texts in Mathematics 52 (1977), Springer-Verlag.

[49] P. Jahnke, I. Radloff, Generatedness for Gorenstein Fano threefolds with canonical singularities, arXiv:math.AG0404156 (2004).

[50] S. Ishii, Quasi-Gorenstein Fano 3-folds with isolated non-rational loci, Comp. Math. 77 (1991), 335341. 
[51] V. Iskovskikh, On birational forms of rational surfaces, Izv. AN SSSR 29 (1965), 1417-1433.

[52] V. Iskovskikh, Rational surfaces with a pencil of rational curves, Mat. Sbornik 74 (1967), 608-638.

[53] V. Iskovskikh, Rational surfaces with a pencil of rational curves with positive square of canonical class, Mat. Sbornik 12 (1970), 91-117.

[54] V. Iskovskikh, Birational properties of a surface of degree 4 in $\mathbb{P}_{k}^{4}$, Mat. Sbornik 17 (1972), 30-36.

[55] V. Iskovskikh, Fano 3-folds I, Izv. AN SSSR 41 (1977), 516-562.

[56] V. Iskovskikh, Fano 3-folds II, Izv. AN SSSR 42 (1978), 504-549.

[57] V. Iskovskikh, Minimal models of rational surfaces over arbitrary fields, Izv. AN SSSR 14 (1980), $17-39$.

[58] V. Iskovskikh, Anticanonical models of three-dimensional algebraic varieties, J. Soviet Math. 13 (1980), 745-814.

[59] V. Iskovskikh, Birational automorphisms of three-dimensional algebraic varieties, J. Soviet Math. 13 (1980), 815-868.

[60] V. Iskovskikh, On the rationality problem for conic bundles, Duke Math. J. 54 (1987), 271-294.

[61] V. Iskovskikh, Lectures on three-dimensional algebraic varieties. Fano varieties, Moscow State University, Moscow (1988).

[62] V. Iskovskikh, Towards the problem of rationality of conic bundles, Lect. Notes Math. 1479 (1991), $50-56$.

[63] V. Iskovskikh, On the rationality problem for conic bundles, Math. USSR, Sb. 72 (1992), 105-111.

[64] V. Iskovskikh, A rationality criterion for conic bundles, Math. USSR, Sb. 187 (1996), 1021-1038.

[65] V. Iskovskikh, Factorization of birational maps of rational surfaces from the viewpoint of Mori theory, Uspekhi Mat. Nauk 51 (1996), 585-652.

[66] V. Iskovskikh, On the rationality problem for three-dimensional algebraic varieties, Proc. Steklov Inst. 218 (1997), 186-227.

[67] V.Iskovskikh, Yu. Manin, Three-dimensional quartics and counterexamples to the Lüroth problem, Mat. Sbornik 86 (1971), 140-166.

[68] V. Iskovskikh, Yu. Prokhorov, Fano varieties, Encyclopaedia Math. Sci. 47 (1999) Springer, Berlin.

[69] A. J. de Jong, N. Shepherd-Barron, A. V. de Ven, On the Burkhardt quartic, Math. Ann. 286 (1990), 309-328.

[70] Yu. Kawamata, A generalization of Kodaira-Ramanujam's vanishing theorem, Math. Ann. 261 (1982), 43-46.

[71] Yu. Kawamata, Crepant blowing-up of 3-dimensional canonical singularities and its application to degenerations of surfaces, Ann. Math. 127 (1988), 93-163.

[72] Yu. Kawamata, K. Matsuda, K. Matsuki, Introduction to the minimal model problem, Adv. Stud. Pure Math. 10 (1987), 283-360.

[73] S. Khashin, Birational automorphisms of the Veronese double cone of dimension three, Vestnik Moscov. Univ., Ser. Mat. Mekh. 1 (1984), 13-16.

[74] J. Kollár, Nonrational hypersurfaces, Journal of the AMS 8 (1995), 241-249.

[75] J. Kollár, Rational curves on algebraic varieties, Springer-Verlag (1996), Berlin.

[76] J. Kollár, Singularities of pairs, Algebraic geometry - Santa Cruz 1995, Proc. Symp. Pure Math. AMS 62 (1997), 221-287.

[77] J. Kollár, Low degree polynomial equations: arithmetic, geometry and topology, Prog. Math. 168 (1998), 255-288.

[78] J. Kollár, Non-rational covers of $\mathbb{C P}^{n} \times \mathbb{C P}^{m}$, L.M.S. Lecture Note Series 281 (2000), 51-71.

[79] J. Kollár et al., Flips and abundance for algebraic threefolds, Astérisque 211 (1992).

[80] Yu. Manin, Rational surfaces over perfect fields, Publ. Math. IHES 30 (1966), 55-114.

[81] Yu. Manin, Rational surfaces over perfect fields II, Mat. Sbornik 72 (1967), 161-192.

[82] Yu. Manin, Cubic forms Nauka (1972).

[83] Yu. Manin, M. Tsfasman, Rational varieties: algebra, geometry, arithmetics, Uspekhi Mat. Nauk 41 (1986), 43-94.

[84] M. Marchisio, Unirational quartic hypersurfaces, Boll. Unione Mat. Ital. 3 (2000), 301-314.

[85] T. Matsusaka, Algebraic deformations of polarized varieties, Nagoya Math. J. 31 (1968), 185-245.

[86] M. Mella, Birational geometry of quartic 3-folds II: the importance of being $\mathbb{Q}$-factorial, Math. Ann. (2004), to appear. 
[87] S. Mori, Threefolds whose canonical bundles are not numerically effective, Ann. Math. 115 (1982), $133-176$.

[88] S. Mori, S. Mukai, Classification of Fano 3-folds with $B_{2} \geq 2$, Manuscr. Mat. 36 (1981), 147-162.

[89] S. Mori, S. Mukai, Erratum, Classification of Fano 3-folds with $B_{2} \geq 2$, Manuscr. Mat. 110 (2003), 407.

[90] D. R. Morrison, The birational geometry of surfaces with rational double points, Math. Ann. 271 (1985), 415-438.

[91] S. Mukai, New developments of Fano varieties - on vector bundles and moduli problems, Sugaku 47 2 (1995), 125-144.

[92] S. Mukai, H. Umemura, Minimal rational threefolds, Lect. Notes in Math. 1016 (1983), 490-518.

[93] Y. Namikawa, Smoothing Fano 3-folds, J. Algebr. Geom. 6 (1997), 307-324.

[94] Yu.Prokhorov, On the degree of Fano 3-folds with canonical Gorenstein singularities, arXiv:math.AG0405347 (2004).

[95] A. Pukhlikov, Birational automorphisms of a double space and a double quartic, Izv. Akad. Nauk SSSR Ser. Mat. 52 (1988), 229-239.

[96] A.Pukhlikov, Birational automorphisms of a three-dimensional quartic with a simple singularity, Mat. Sbornik 177 (1988), 472-496.

[97] A. Pukhlikov, Maximal singularities on the Fano variety $V_{6}^{3}$, Moscow Univ. Math. Bull. 44 (1989), $70-75$.

[98] A. Pukhlikov, Birational automorphisms of double spaces with sigularities, J. Math. Sci. 85 (1997), $2128-2141$.

[99] A. Pukhlikov, Birational automorphisms of three-dimensional algebraic varieties with a pencil of del Pezzo surfaces, Izv. Math. 62 (1998), 115-155.

[100] M. Reid, Projective morphism according to Kawamata, preprint (1983), University of Warwick.

[101] M. Reid, Canonical 3-folds, Journées de Géometrie Algébrique d'Angers (1979), 273-310, Sijthoff \& Noordhof.

[102] M. Reid, Chapters on algebraic surfaces, Complex algebraic geometry (J.Kollár, editor), Lecture notes from a summer program held in Park City, Utah, in 1993 (1997), 5-159.

[103] L. Roth, Algebraic threefolds with special regard to problems of rationality (Springer-Verlag, Berlin, 1955).

[104] B. Saint-Donat, Projective models of K-3 surfaces, Amer. J. Math. 96 (1974), 602-639.

[105] T. Sano, Classification of non-Gorenstein $\mathbb{Q}$-Fano d-folds of Fano index greater than $d-2$, Nagoya Math. J. 142 (1996), 133-143.

[106] B. Segre, Variazione continua ad omotopia in geometria algebrica, Ann. Mat. Pura et Appl. 50 (1960), 149-186.

[107] V. Shokurov, Theorem of Nöether-Enriques on canonical curves, Mat. Sbornik 86 (1971), 367-408.

[108] V.Shokurov, Smoothness of the general anticanonical divisor on Fano 3-fold, Izv. AN SSSR 43 (1979), 430-441.

[109] V. Shokurov, Existence of a line on a Fano 3-fold, Izv. AN SSSR 43 (1979), 922-964.

[110] V. Shokurov, Prym varieties: theory and applications, Math. USSR, Izv. 23 (1984), 93-147.

[111] J. Stevens, On canonical singularities as total spaces of deformations, Abh. Math. Sem. Univ. Hamburg $\mathbf{5 8}$ (1988), 275-283.

[112] A. Tikhomirov, Geometry of the Fano surface of a double $P^{3}$ branched in a quartic, Izv. Akad. Nauk SSSR Ser. Mat. 44 (1980), 415-442.

[113] A. Tikhomirov, The intermediate Jacobian of double $P^{3}$ that is branched in a quartic, Izv. Akad. Nauk SSSR Ser. Mat. 44 (1980), 1329-1377.

[114] A. Tikhomirov, Singularities of the theta-divisor of the intermediate Jacobian of the double $P^{3}$ of index two, Izv. Akad. Nauk SSSR Ser. Mat. 46 (1982), 1062-1081.

[115] A. Tikhomirov, The Abel-Jacobi mapping of sextics of genus three onto double $P^{3}$ 's of index two, Dokl. Akad. Nauk SSSR 286 (1986), 821-824.

[116] M. Tsfasman, Arithmetic of singular del Pezzo surfaces, Uspekhi Mat. Nauk 38 (1983) 131-132.

[117] A. Tyurin, On the intersection of quadrics, Uspekhi Mat. Nauk 30 (1975), 51-99.

[118] A. Tyurin, The middle Jacobian of three-dimensional varieties, J. Soviet Math. 13 (1980), 707-745.

[119] V. Viehweg, Vanishing theorems, J. Reine Angew. Math. 335 (1982), 1-8. 
[120] C. Voisin, Hodge theory and complex algebraic geometry II, Cambridge Studies in Advanced Mathematics 77 (Cambridge Univ. Press, 2003).

Steklov Institute of Mathematics, 8 Gubkin street, Moscow 117966, Russia

E-mail address: cheltsov@yahoo.com, shramov@mccme.ru, przhijal@mccme.ru 